\title{
The role of astrocytes for oligodendrocyte death and remyelination
}

\author{
Dissertation \\ for the award of the degree \\ “Doctor rerum naturalium” (Dr. rer. nat.)
}

Division of Mathematics and Natural Sciences

Georg August University Göttingen

submitted by

Claudia Wrzos

from Pszczyna (Pleß), Poland

Göttingen, 2012 


\section{Thesis Committee}

\section{$1^{\text {st }}$ Reviewer}

Prof. Dr. Christine Stadelmann-Nessler

Institute of Neuropathology

University Medical Center Göttingen

Georg August University Göttingen

\section{$2^{\text {nd }}$ Reviewer}

Prof. Dr. Michael Müller

Department of Neuro- and Sensory Physiology University Medical Center Göttingen Georg August University Göttingen

\section{$3^{\text {rd }}$ Member of the Thesis Committee}

\section{Prof. Dr. Dr. Hannelore Ehrenreich}

Department of Clinical Neuroscience

Max Planck Institute for Experimental Medicine Göttingen

Date of oral examination: March $6^{\text {th }}, 2012$ 
Dla maich radziców 


\section{Declaration}

I hereby declare that I have written my Ph.D. thesis entitled "The role of astrocytes for oligodendrocyte death and remyelination" independently and with no other sources and aids than quoted. This thesis has not been submitted elsewhere for any academic degree.

Claudia Wrzos

Göttingen, January 2012 


\section{Abstract}

Astrocytes are the most abundant cells in the central nervous system (CNS) with wide-ranging functions.

The first part of my thesis deals with Neuromyelitis optica (NMO), an inflammatory demyelinating disease of the CNS. Aquaporin 4 (AQP4), a water channel expressed in high density at perivascular astrocytic endfeet, has recently been identified as a target of the humoral immune response in NMO. I developed two in vivo models to assess the extent, time course, and mechanisms of tissue damage and repair after astrocyte depletion. A patient derived, recombinant anti-AQP4 ( $\mathrm{rAb}-53$ ) antibody was i.v. transferred into immunized rats (experimental autoimmune encephalomyelitis (EAE)/NMO model) or focally injected into the rat cerebral cortex (focal NMO model). One hour after a single cerebral injection of complement-binding rAb-53, a selective depletion of astrocytes was found. 3hrs after antibody injection, in addition, a diminution of oligodendrocytes was observed. 24hrs after antibody injection the astrocyte depleted areas reached their full extent and revealed loss of oligodendrocytes and oligodendrocyte precursor cells (OPCs). After two weeks, astrocytic repopulation of the lesion was nearly complete. However, the prelesional density of oligodendrocytes was not yet achieved. To assess the role of excess glutamate and ATP on oligodendroglial cell death, N-methyl D-aspartate (NMDA) and $\mathrm{P}_{2} \mathrm{X}_{7}$ receptor antagonists were applied in vivo and in vitro. However, no clear-cut effect of NMDA and $\mathrm{P}_{2} \mathrm{X}_{7}$ receptor antagonists on oligodendroglial cell death was observed. In the systemic EAE/NMO model, administration of rAb-53 to preimmunized rats demonstrated huge perivascular astrocyte depleted areas in the spinal cord accompanied by significant loss of oligodendrocytes and OPCs after 30hrs. In addition, kidney pathology with detached cells of the inner medullary collecting ducts, where AQP4 is expressed, and infiltrating macrophages were observed.

In summary, both models successfully mimicked human NMO lesions. The human rAb-53 was able to induce astrocyte depletion with a single cerebral injection or when administered to immunized rats. Activation of the complement system was found to be a prerequisite for astrocyte lysis. Furthermore, this work demonstrates that oligodendroglial cell death follows astrocyte depletion quite rapidly. However, 
oligodendroglial repair of astrocyte depleted lesions is slow compared to classical demyelinated lesions.

In the second part of my thesis the role of fibroblast growth factor 9 (FGF9) on demyelination (DM) and remyelination (RM) was assessed. To provide overexpression of this growth factor in vivo, an adeno-associated virus 6 (AAV-6)based vector containing the cDNA of FGF9 under the control of the GFAP promoter was constructed. This FGF9-AAV-6-based vector was intracerebrally injected in cuprizone-fed mice and rats with focal EAE to study a possible role of FGF9 on DM and RM. In both models, no clear effect of FGF9 on RM was found. However, in both models FGF9 led to widespread activation and proliferation of astrocytes and similarly, activation and proliferation of oligodendroglia.

Thus, my results indicate that FGF9 has an important role for the homeostasis of glial cells, however, rather influences cell number and activation state than repair. 


\section{Contents}

ABSTRACT

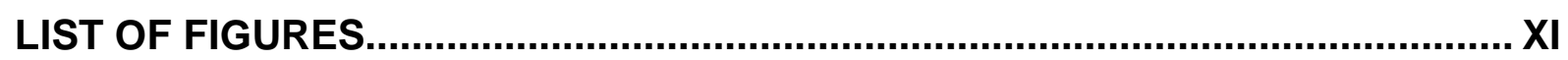

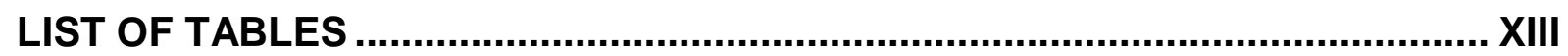

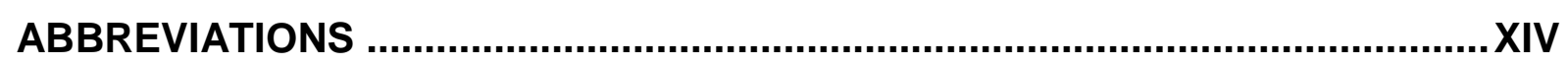

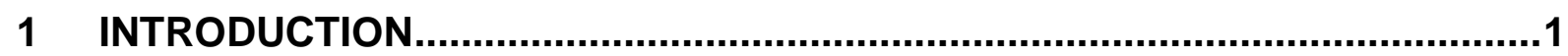

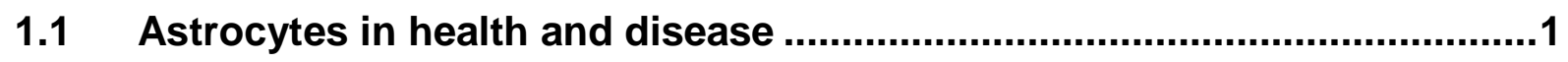

1.1.1 Physiological functions of astrocytes in the healthy CNS ........................

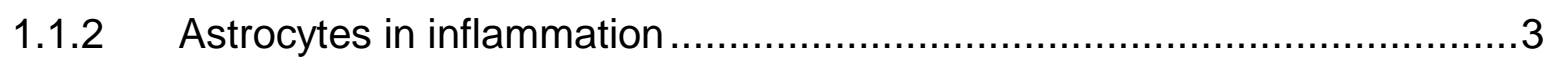

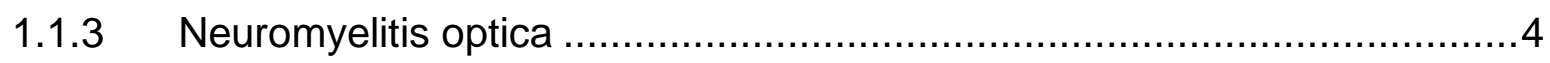

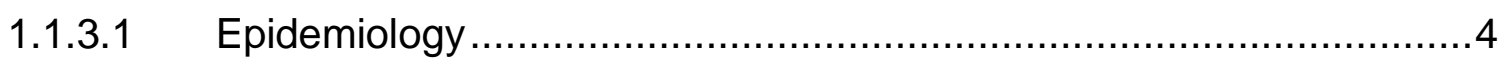

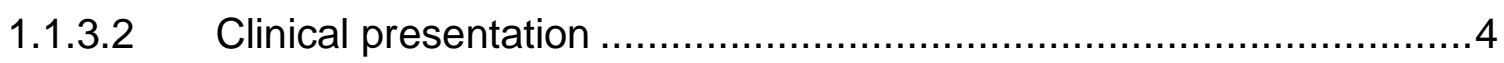

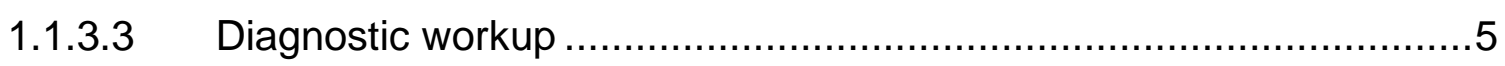

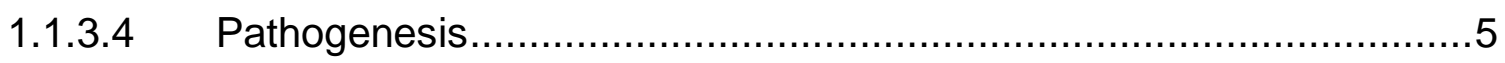

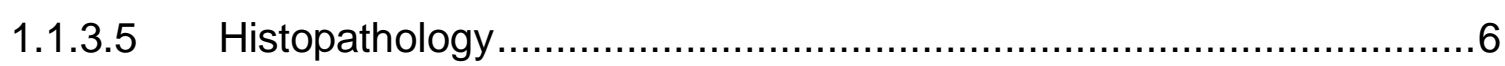

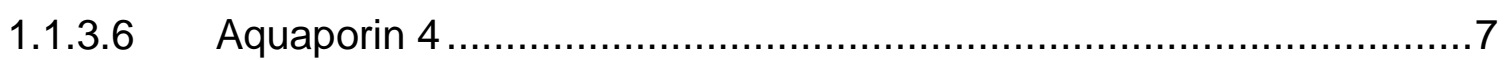

1.1.4 Effect of astrocytic overexpression of fibroblast growth factor 9 (FGF9)

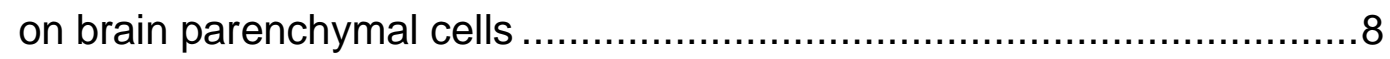

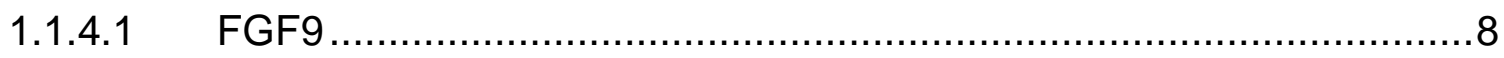

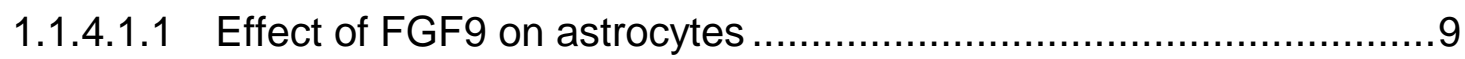

1.1.4.1.2 Effect of FGF9 on oligodendrocytes and OPCs ............................

1.1.4.1.3 Effect of FGF9 on remyelination (RM) ......................................

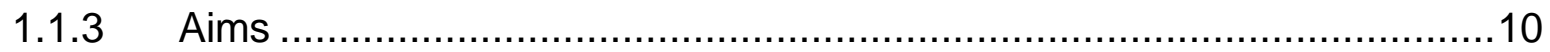

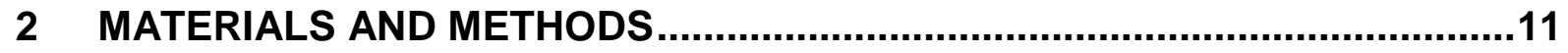

$2.1 \quad$ Animals

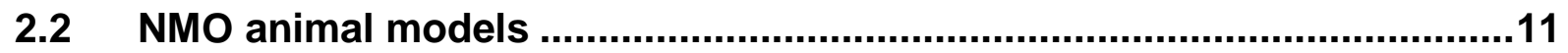

2.2.1 Generation of focal NMO-like lesions in vivo.......................................11

2.2.1.1 Intracerebral stereotactic injection ........................................12

2.2.1.2 Pharmacological experiments to determine the role of NMDA and

$\mathrm{P}_{2} \mathrm{X}_{7}$ receptors in oligodendrocyte death....................................12

2.2.2 I.v. transfer of $\mathrm{rAb}-53$ into animals with experimental autoimmune encephalomyelitis (EAE) ................................................................. 13

2.2.2.1 Sensitization procedure ……………….................................13 


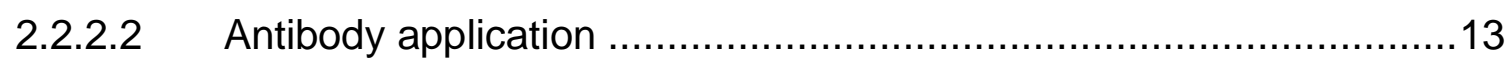

2.2.2.3 Kidney function parameters ....................................................13

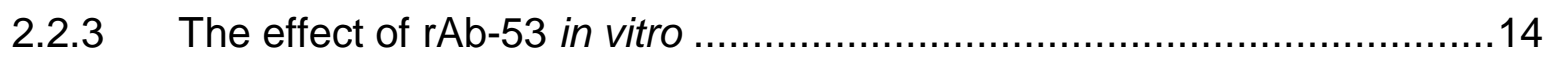

2.2.3.1 Isolation of primary glial cells from newborn animals ......................14

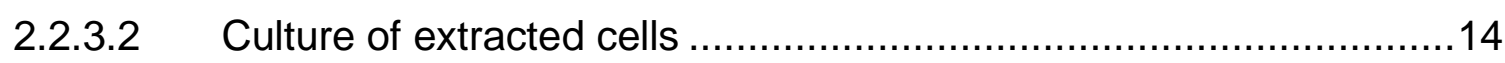

2.2.3.3 Pharmacological experiments to determine the role of NMDA and $\mathrm{P}_{2} \mathrm{X}_{7}$ receptors for oligodendrocyte death in vitro...........................15

2.2.3.3.1 Cell viability assay (WST-1) .................................................. 16

2.2.3.3.2 CytotoxOne membrane integrity assay .....................................16

2.3 Overexpression of FGF9 in demyelinating animal models.....................17

2.3.1 Adeno-associated viral 6 (AAV-6)-based vector as a tool to express

FGF9 in astrocytes .................................................................... 17

2.3.1.1 Injection of viral vectors in focal EAE ......................................19

2.3.1.1.1 Enzyme-linked immunosorbent assay (ELISA) ..........................20

2.3.1.1.2 Induction of focal EAE lesions and intracerebral viral vector

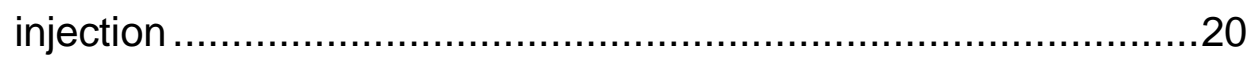

2.3.1.2 Injection of viral vectors in the cuprizone model............................20

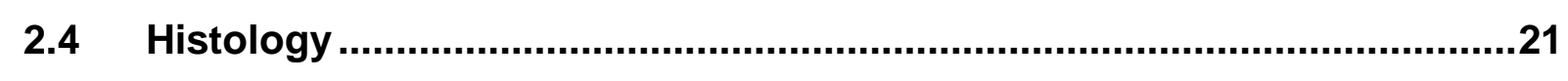

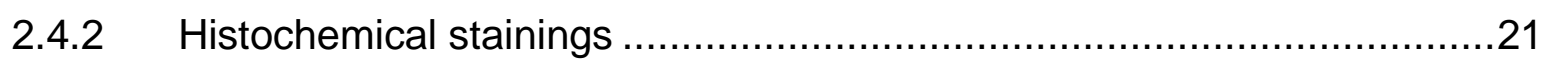

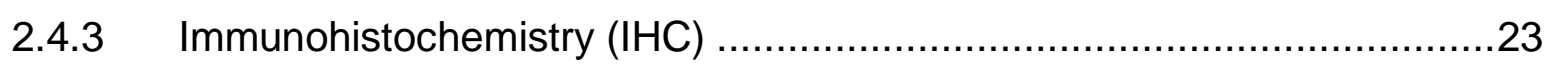

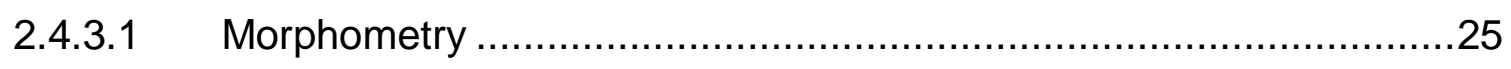

2.4.3.2 GFAP/NogoA/Olig2 triple immunofluorescence .............................26

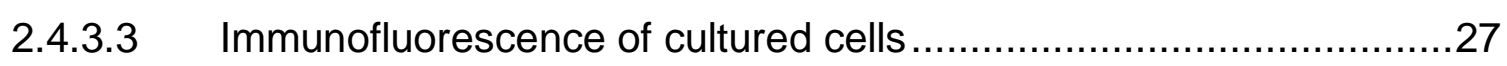

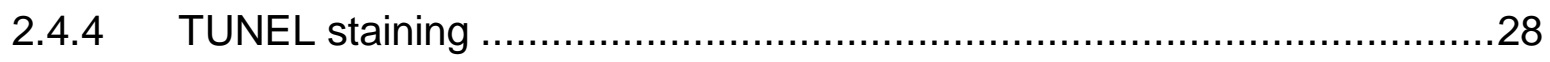

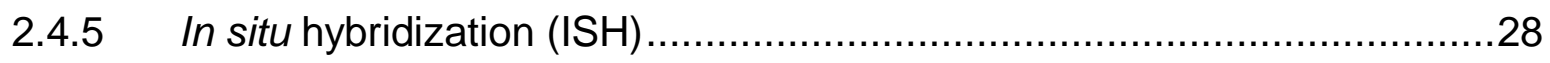

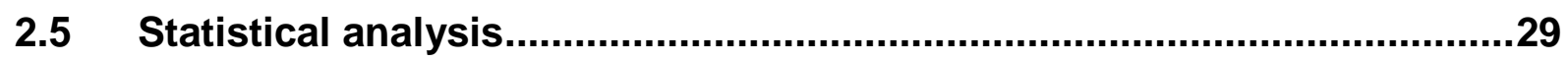

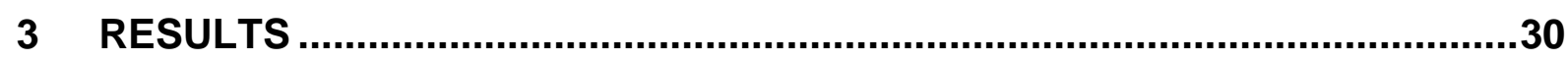

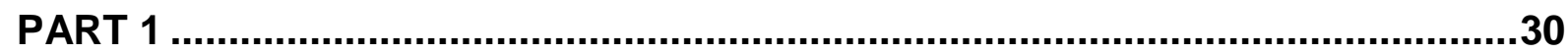

3.1 Modeling Neuromyelitis optica (NMO) in vitro and in vivo .......................30

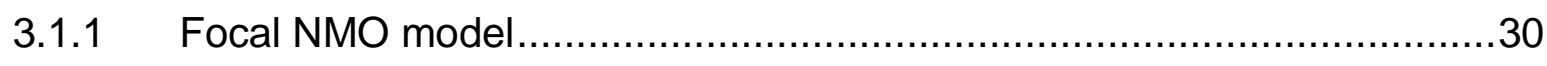

3.1.1.1 Astrocyte depletion by $\mathrm{rAb}-53$ antibodies complement dependent...30

3.1.1.2 Features of astrocyte depleted areas in the focal NMO model.........31

3.1.1.3 Astrocyte death precedes oligodendroglial demise ........................32

3.1.1.4 Time course of inflammatory cell infiltration ....................................33

3.1.1.5 Rapid repopulation of astrocyte depleted focal brain lesions ...........34 
3.1.1.6 Little oligodendrocyte repopulation and myelin repair one week

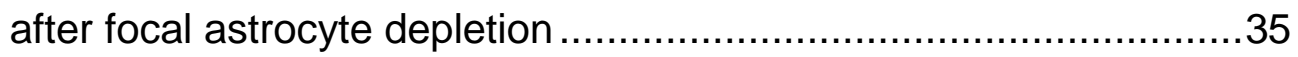

3.1.1.7 Mechanisms of oligodendroglial cell death in vivo ..........................36

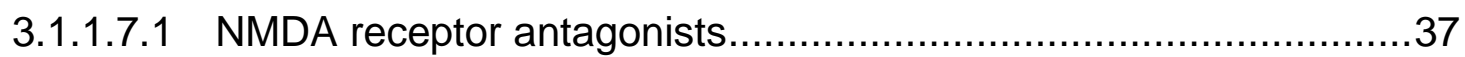

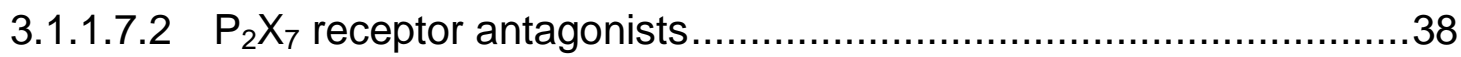

3.1.2 Antibody-transfer model of NMO (EAE/NMO model) ............................39

3.1.2.1 Features of astrocyte depleted areas in rat EAE .............................39

3.1.2.2 Reduction of mature oligodendrocytes in astrocyte depleted areas .41

3.1.2.3 Loss of PLP mRNA positive oligodendrocytes in astrocyte

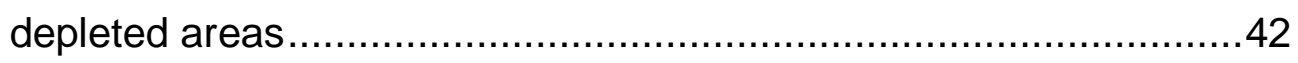

3.1.2.4 OPCs are as vulnerable as mature oligodendrocytes .....................43

3.1.2.5 Activated caspase-3 positive oligodendrocytes in astrocyte depleted areas.

3.1.2.6 Kidney pathology in the EAE/NMO model ..................................46

3.1.2.6.1 Macrophage infiltration in the kidneys......................................4

3.1.2.6.2 Cell debris and protein content of the urine ...............................48

3.1.2.7 Mutants of $\mathrm{rAb}-53$ show different extent of histopathology in the EAE/NMO model

3.1.3 Effect of $\mathrm{rAb}-53$ on astrocytes and oligodendrocytes in vitro..................51

3.1.3.1 Assessment of mechanisms of oligodendroglial cell death in vitro ...54

3.1.3.1.1 NMDA and $\mathrm{P}_{2} \mathrm{X}_{7}$ receptor antagonists ...................................54

PART 2

3.2 Effect of astrocytic overexpression of FGF9 .......................................56

3.2.1 Astrocyte and oligodendroglial phenotype after intracerebral

FGF9-AAV-6 -based vector injection in the cuprizone mouse model ......57

3.2.2 Astrocyte and oligodendroglial phenotype after intracerebral

FGF9-AAV-6-based vector injection in focal EAE ..............................61

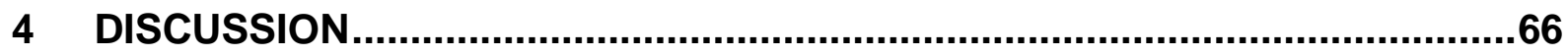

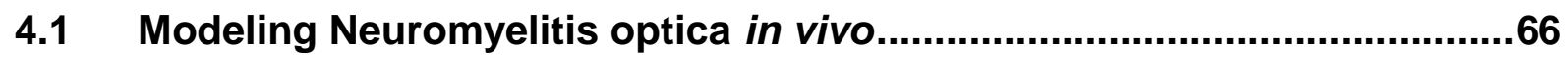

4.1.1 Mechanism of oligodendroglial cell death in vivo and in vitro .................68

4.1.2 Kidney pathology in the EAE/NMO model..........................................69

4.2 Effect of astrocytic overexpression of FGF9 ......................................70

4.2.1 Overexpression of FGF9 in the cuprizone mouse model of toxic DM and in a model of antibody-mediated focal inflammatory DM in the rat ...71

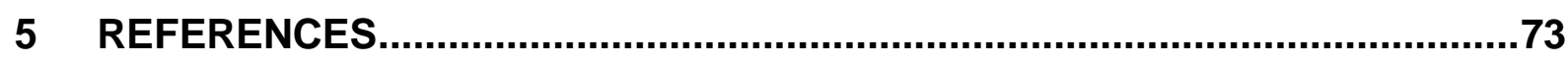




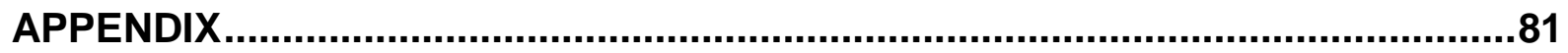

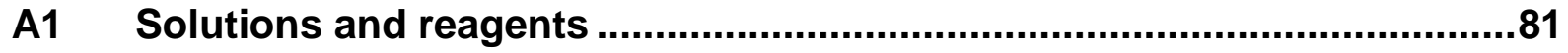

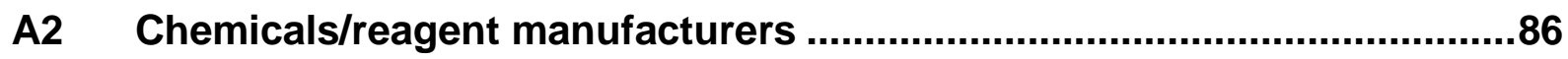

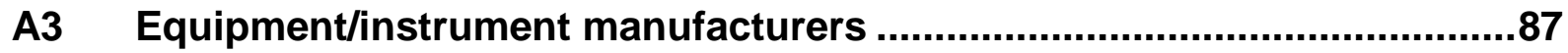

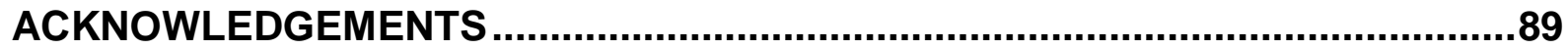

CURRICULUM VITAE .....................................................................................90 


\section{List of figures}

Figure 1.1: Schematic summary of astrocyte functions

Figure 1.2: NMO lesion pathology in a 77 year old woman seropositive for anti-AQP4 antibodies

Figure 1.3: Model of AQP4

Figure 2.1: Principle of the WST-1 assay 16

Figure 2.2: Principle of the CytotoxOne membrane integrity assay 17

Figure 2.3: Vector card of the control AAV-6 with EGFP insertion constructed by Sebastian Kügler

Figure 2.4: Vector card of the FGF9 containing AAV-6 modified by Andreas Junker

Figure 2.5: Principle of the tyramide signal amplification method

Figure 3.1: Complete astrocyte loss in the cortex of a focally injected rat with rAb-53 and human serum (A; GFAP), but not with rAb-53 alone (B; GFAP) 24hrs after injection

Figure 3.2: Histopathological features of astrocyte depleted areas in the focal NMO model $24 \mathrm{hrs}$ after injection of $\mathrm{rAb}-53$ with human serum

Figure 3.3: Early evolution of focal NMO-like lesions

Figure 3.4: Time course of ED1 positive macrophages/activated microglia in focal NMO-like lesions

Figure 3.5: Time course of granulocytes in focal NMO-like lesions

Figure 3.6: Focal NMO-like lesions are in part repopulated by astrocytes already one week after $\mathrm{rAb}-53$ injection

Figure 3.7: Time course of DM and oligodendrocyte repopulation

Figure 3.8: Massive loss of oligodendrocytes and OPCs is observed in animals focally injected with $r A b-53$ and human serum

Figure 3.9: NMDA receptor antagonist treatment in focal NMO-like lesions 38

Figure 3.10: $\mathrm{P}_{2} \mathrm{X}_{7}$ receptor antagonist treatment in focal $\mathrm{NMO}$-like lesions

Figure 3.11: Astrocyte depletion in the rat EAE model when i.v. injected with rAb-53 $(A+C)$, but not with control antibody $(B+D)$

Figure 3.12: Histopathological features of astrocyte depleted areas in the $\mathrm{NMO} / \mathrm{EAE}$ model $30 \mathrm{hrs}$ after rAb-53 administration 
Figure 3.13: NMO-like lesions reveal a decrease in oligodendrocytes 30hrs after antibody administration

Figure 3.14: Reduction of PLP mRNA positive cells in EAE/NMO lesions

Figure 3.15: Olig2 positive cells are decreased in NMO-like lesions 30hrs after rAb-53 injection

Figure 3.16: GFAP/Olig2/NogoA triple immunohistochemistry confirms the decrease of OPCs in NMO-like lesions

Figure 3.17: Oligodendrocyte death is caspase-3 dependent

Figure 3.18: AQP4 staining (brown) of inner medullary collecting ducts (IMCDs) in the EAE/NMO model

Figure 3.19: Increased macrophage infiltration in kidneys

Figure 3.20: Pappenheim/Giemsa staining of a urinary cytospin collected 30hrs after i.v. injection of rAbs

Figure 3.21: The percentage of astrocyte depleted spinal cord area

Figure 3.22: Numbers of mature oligodendrocytes in EAE/NMO in white and grey matter lesions

Figure 3.23: Astrocyte cell culture

Figure 3.24: LDH (cytotoxicity) assay of representative astrocyte cultures

Figure 3.25: Oligodendrocyte cell cultures

Figure 3.26: LDH (cytotoxicity) assay of representative oligodendrocyte cultures

Figure 3.27: Expression of FGF9 and EGFP on day 10

Figure 3.28: Hemispheric frontal brain sections of mice injected with PBS (A-D), EGFP-AAV-6-based vector (E-H) or FGF9-AAV-6-based vector $(\mathrm{I}-\mathrm{L})$

Figure 3.29: Hemispheric frontal brain sections of mice injected with PBS, EGFP-AAV-6-based vector (C-D) or FGF9-AAV-6-based vector $(\mathrm{E}-\mathrm{F})$ in the cuprizone model

Figure 3.30: Hemispheric frontal brain sections of mice injected with PBS (A-D), EGFP-AAV-6-based vector (E-H) or FGF9-AAV-6-based vector $(\mathrm{I}-\mathrm{L})$ in the cuprizone model

Figure 3.31: Brain sections of mice injected with PBS, EGFP-AAV-6-based vector (C-D) or FGF9-AAV-6-based vector (E-F) in the cuprizone model 
Figure 3.32: 10 days after EGFP-AAV-6-based vector (A, B) or FGF9-AAV-6-based vector $(C, D)$ injections in naïve rats

Figure 3.33: Rat focal EAE 3 days after injection of cytokines alone $(A)$, cytokines with EGFP-AAV-6-based vector $(C)$ and cytokines with FGF9-AAV-6-based vector (E)

Figure 3.34: Rat focal EAE 10 days after injection of cytokines $(A-F)$, cytokines with EGFP-AAV-6-based vector (G-L) and cytokines with FGF9-AAV-6-based vector (M-R)

Figure 3.35: Astrocytic and oligodendroglial proliferation in the rat focal EAE model after intracerebral injection with cytokines and FGF9-AAV-6based vector

Figure 3.36: Rat focal EAE 10 days after injection of FGF9-AAV-6-based vector alone

\section{List of tables}

Table 2.1: $\quad$ EAE score on scale 0 to 5

Table 2.2: $\quad$ List of primary antibodies 


\section{Abbreviations}

ANOVA

AAV

$\mathrm{Ab}$

ADCC

AP

APAAP

APP

AQP

ATP

BBB

BBG

BCIP

BME

BrdU

BSA

C

$\mathrm{Ca}$

CNP

CNS

CSF

Cy

DAB

D-AP5

DAPI

DM

DMEM

DNA

dUTP

EAE

EAAT

EDTA

FCS analysis of variance

Adeno-associated virus

antibody

antibody-dependent cell-mediated cytotoxicity

alkaline phosphatase

alkaline phosphatase anti-alkaline phosphatase

amyloid precursor protein

aquaporin

adenosine triphosphate

blood-brain barrier

brilliant blue $\mathrm{G}$

5-bromo-4-chloro-3-indolyl phosphate

basal medium eagle

5-bromo-2-deoxyuridine

bovine serum albumin

Celsius

calcium

2',3'-cyclic nucleotide 3'-phosphodiesterase 1

central nervous system

cerebrospinal fluid

indocarbocyanine

3,3'-diaminobenzidine tetrachloride

D-2-Amino-5-phosphonopentanoic acid

4',6-diamidino-2-phenylindole

demyelination

Dulbecco's modified Eagle medium

deoxyribonucleic acid

deoxyuridine triphosphate

experimental autoimmune encephalomyelitis

excitatory amino acid transporter

ethylenediaminetetraacetic acid

fetal calf serum 


\begin{tabular}{|c|c|}
\hline FGF & fibroblast growth factor \\
\hline GABA & y-Aminobutyric acid \\
\hline GFAP & glial fibrillary acidic protein \\
\hline GM & grey matter \\
\hline HBSS & Hank's buffered salt solution \\
\hline $\mathrm{H} \& \mathrm{E}$ & hematoxylin and eosin \\
\hline HRP & horseradish peroxidase \\
\hline IFA & incomplete Freund's adjuvant \\
\hline IFN & interferon \\
\hline IGF & Insulin derived growth factor \\
\hline $\lg G$ & immunoglobulin G \\
\hline $\mathrm{IHC}$ & immunohistochemistry \\
\hline IMCD & inner medullary collecting duct \\
\hline $\mathrm{K}$ & potassium \\
\hline IL & interleukin \\
\hline i. p. & Intraperitoneal \\
\hline ISH & in situ hybridization \\
\hline i. v. & intravenous \\
\hline $\mathrm{LDH}$ & lactate dehydrogenase \\
\hline LETM & longitudinally extensive transverse myelitis \\
\hline LFB & luxol fast blue \\
\hline M1 & primary motor cortex \\
\hline MAG & myelin-associated glycoprotein \\
\hline MBP & myelin-basic protein \\
\hline MCP & monocyte chemoattractant protein \\
\hline $\mathrm{MHC}$ & major histocompatibility complex \\
\hline MK-801 & (+) MK-801 hydrogen maleate \\
\hline MMP & matrix metalloprotease \\
\hline MOG & myelin oligodendrocyte glycoprotein \\
\hline MS & multiple sclerosis \\
\hline $\mathrm{Na}$ & sodium \\
\hline$N F-\kappa b$ & nuclear factor kappa b \\
\hline NMDA & N-methyl D-aspartate \\
\hline NMO & neuromyelitis optica \\
\hline
\end{tabular}


NogoA

NT

OCB

Olig2

OPC

OATP

PAS

PBS

PDGF

Pen/Strep

PFA

PLL

PLP

PMN

PRR

$\mathrm{RM}$

rMOG

SC

TBS

TLR

TNF

Tris

Tris-EDTA

TSA

TUNEL

WM

WPRE

WST

ZTE neurite outgrowth inhibitor $A$

Neurotrophin

oligoclonal bands

oligodendrocyte transcription factor 2

oligodendrocyte precursor cell

periodate oxidized adenosine

periodic acid-Schiff

phosphate buffered saline

platelet derived growth factor

penicillin/streptomycin

paraformaldehyde

poly-L-lysin

proteolipid protein

polymorphnuclear cells

pattern recognition receptor

remyelination

recombinant myelin oligodendrocyte glycoprotein

spinal cord

Tris buffered saline

Toll like receptor

tumor necrosis factor

tris(hydroxymethyl)aminomethane

tris-ethylenediaminetetraacetic acid

tyramide signal amplification

terminal deoxynucleotidyl transferase dUTP nick end labeling

white matter

woodchuck hepatitis post transcriptional control element

water soluble tetrazolium

Zentrale Tierexperimentelle Einrichtung 
Introduction

\subsection{Astrocytes in health and disease}

Astrocytes, first characterized by Camillo Golgi in 1871, are the most abundant cells in the central nervous system (CNS) and outnumber neurons at least fivefold (He and Sun, 2007). These glia cells were first regarded as the "glue" that holds the CNS together but multiple additional functions of astrocytes have been described since then. Astrocytes participate in the regulation of blood flow and control fluid, ion and neurotransmitter homeostasis. They modulate synaptic activity and are relevant as energy suppliers (Fig. 1.1). Besides their essential contributions in the healthy CNS, astrocytes contribute to a variety of CNS disorders and pathologies.

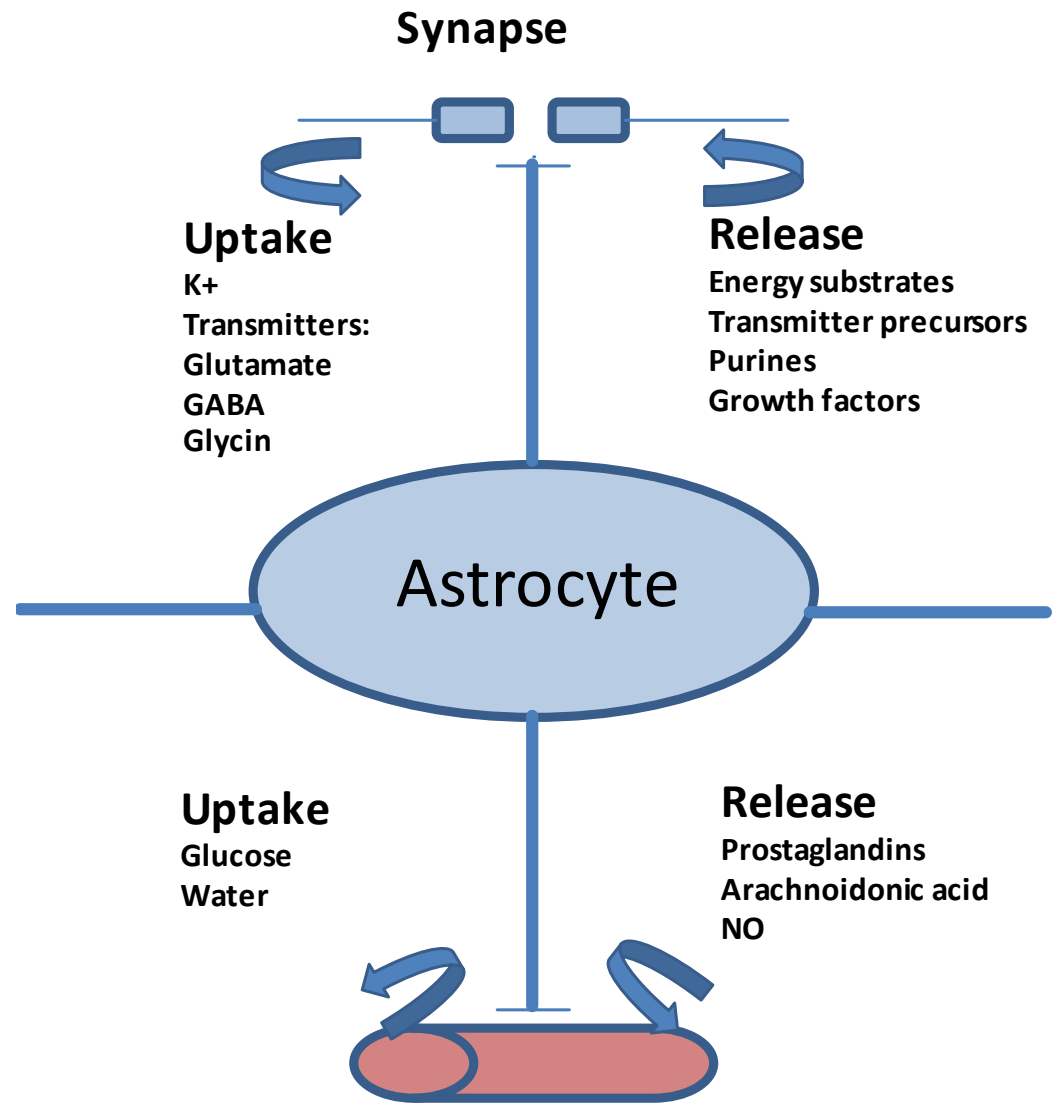

Blood vessel

Figure 1.1: Schematic summary of astrocyte functions. Adapted from Sofroniew (2010). 


\subsubsection{Physiological functions of astrocytes in the healthy CNS}

Regulation of blood flow: Astrocytes have extensive contact with blood vessels and synapses and as such form neuro-glial-vascular domains. They can release mediators such as prostaglandins, nitric oxide or arachidonic acid, which can influence the blood vessel diameter. Blood flow can also be regulated in response to synaptic activity (Zonta et al., 2003).

Regulation of ion, fluid and transmitter homeostasis: Astrocytic processes enwrap all synapses and express multiple transporters which are essential for synaptic function. Astrocytes clear the synaptic cleft from $\mathrm{K}^{+}$, neurotransmitters (glutamate, GABA and glycine) or protons $\left(\mathrm{Na}^{+} / \mathrm{H}^{+}\right.$exchanger). Astrocytic networks connected by gap junctions might prevent the potential toxicity of glutamate by the rapid dilution of this molecule. Aquaporin 4 (AQP4) water channels localized at astrocytic processes which contact blood vessels are important for fluid homeostasis.

Modulation of synaptic activity: Astrocytes can modulate synaptic activity by the release of transmitters (glutamate, GABA, ATP) and by releasing co-factors such as D-serine (Mothet et al., 2000; Schell et al., 1995). Astrocytes are also instrumental during synapse development.

Energy metabolism: Astrocytes are the main glycogen storage sites in the CNS and support neurons in periods of high neuronal activity or hypoglycemia.

Blood-brain barrier (BBB): The BBB is composed of endothelial cells connected by tight junctions, the basal lamina, perivascular pericytes and astrocyte end-feet. Astrocytes and basal lamina constitute the glia limitans and restrict - in combination with the endothelial cells - diffusion of molecules and entry of leukocytes into the CNS.

Astrocytes participate in myelination by the secretion of diverse growth factors. Platelet-derived growth factor (PDGF), which promotes the proliferation, migration and differentiation of oligodendrocyte precursor cells (OPCs), is synthesized by neurons and astrocytes (Gard et al., 1995). Astrocytes are the source of insulin-like 
growth factor (IGF), an oligodendrocyte mitogen, differentiation and survival factor. They release neurotrophin-3 (NT-3) and basic fibroblast growth factor (bFGF or FGF2), which support proliferation and survival of oligodendrocyte precursors (Dreyfus et al., 1999).

\subsubsection{Astrocytes in inflammation}

Astrocytes and microglia cells are components of the innate immune system in the CNS, which protect the host by distinguishing molecular structures that are normally absent in the healthy organism. Effective detection of foreign (microbial) or modified endogenous ligands depends on pattern recognition receptors (PRR) such as scavenger or Toll-like receptors (TLR) which recognize conserved non-self motifs (Medzhitov, 2007). Astrocytes express TLR3 in vivo (Kim et al., 2006) and respond to TLR3 ligation with the synthesis of proinflammatory mediators such as IL-6, CXCL10 and INF- $\beta$ (Jack et al., 2005) and potentially neuroprotective anti-inflammatory cytokines such as IL-10 or IL-11 (Bsibsi et al., 2006). PRR-mediated activation of astrocytes might therefore amplify the local innate immune response (IL-1, IL-8), open the BBB (IL-6; MCP-1) and attract leukocytes from the blood into the CNS (IL-8, MCP-1, RANTES). On the other hand, astrocytes respond to TLR3 ligation by producing immunosuppressive cytokines and mediators of tissue repair. Keeping the balance between inflammatory and immunosuppressive pathways is essential for the response of astrocytes to tissue damage. This is probably best illustrated in a number of animal models, where astrocyte functions are partially or completely inhibited. Selective inactivation of NF-kb (Nuclear Factor kappa b) in astrocytes improves spinal cord injury (Brambilla et al., 2005) and inactivation of the upstream NF-kb inactivators NEMO or IKK2 in CNS cells improves EAE (van Loo et al., 2006). Complete ablation of proliferating astrocytes by ganciclovir in animals which express the herpes simplex virus thymidine kinase under the glial fibrillary acidic protein (GFAP) promoter is, however, detrimental and results in more severe and longstanding inflammation, BBB dysfunction and neuronal degeneration (Bush et al., 1999; Faulkner et al., 2004). 


\subsubsection{Neuromyelitis optica}

Neuromyelitis optica (NMO), first described by Clifford Allbutt in 1870 (Allbutt, Lancet 1870, p.203) and summarized by Eugene Devic in 1894 (Wingerchuk et al., 1999), is an idiopathic inflammatory demyelinating disease of the CNS. Previously thought to be a variant of multiple sclerosis (MS), distinctive features of NMO have been described over the last decade. The most important finding was the discovery of an NMO-lgG which targets AQP4 in 70\% of the patients (Lennon et al., 2005; Lennon et al., 2004).

\subsubsection{Epidemiology}

NMO is a rare disorder with a comparable incidence of $0.4 / 10^{5}$ in non-white and white populations. It is three to nine times more common in women than men in patients with relapsing disease, whereas patients suffering from monophasic NMO do not have any sex bias (Wingerchuk et al., 1999). The median age of disease onset is the late thirties (Barbieri and Buscaino, 1989; Davis et al., 1996; Wingerchuk et al., 1999).

\subsubsection{Clinical presentation}

NMO preferentially affects the optic nerves and the spinal cord (Wingerchuk et al., 2007a; Wingerchuk et al., 2007b) while brain regions are spared in early stages (Wingerchuk et al., 1999). NMO shows a relapsing disease course in two-thirds (Mandler et al., 1998; Wingerchuk et al., 1999) and the development of permanent disability is attack-related. Relapses can be separated by years or even decades (Wingerchuk et al., 2007b). Patients usually complain about ocular pain followed by loss of vision or symptoms characteristic of severe myelitis such as symmetric paraplegia, sensory loss and bladder dysfunction (Wingerchuk and Weinshenker, 2003). Attacks generally worsen in the first week, and recovery in NMO is incomplete. 


\subsubsection{Diagnostic workup}

The diagnosis of NMO is based on clinical criteria in conjunction with radiological and serological testings. The main clinical features are optic neuritis and longitudinally extensive transverse myelitis (LETM). Supportive criteria are a brain magnetic resonance imaging (MRI) atypical for multiple sclerosis (Gard et al., 1995), a spinal MRI showing that the spinal cord lesion extends over three or more vertebrae and anti-AQP4 seropositivity (Wingerchuk et al., 2006; Wingerchuk and Weinshenker, 2008). In the cerebrospinal fluid a mixed lymphocytic and neutrophilic pleocytosis is typical, and oligoclonal bands are detectable in $35 \%$ of the cases (Ghezzi et al., 2004; Jarius et al., 2008).

\subsubsection{Pathogenesis}

NMO is currently considered to be an autoimmune disease with auto-antibodymediated injury to astrocytes. The target is the auto-antigen AQP4, which is a water channel present in high concentrations in astrocytes of the spinal cord, diencephalon and the periventricular zone. Immunoglobulins of anti-AQP4 antibody-positive patients or recombinant antibodies against AQP4 are pathogenic if transferred to animals in which the BBB has been opened by activated CNS-specific $T$ cells (Bennett et al., 2009; Bradl et al., 2009). The frequency of familial NMO is higher than expected and argues for a genetic component of disease susceptibility. HLA and mitochondrial genes have been analyzed as contributors. HLA-DRB1*1501 is the allele most strongly associated with genetic susceptibility to MS, but has not been associated with NMO, according to a number of small studies (Brum et al., 2010; Cree et al., 2009; Zephir et al., 2009). In a Japanese population, HLA-DPB1*0501 was more frequent in patients with NMO, but HLA-DRB1*1501 was also not (Matsushita et al., 2009). Genetic variations of AQP4 also failed to account for the overall susceptibility to NMO (Matiello et al., 2011). A single common nucleotide polymorphism in the promoter of CYP7A1, which codes for cytochrome P450 was found to be protective (Kim et al., 2010). 


\subsubsection{Histopathology}

The most important distinguishing histopathological feature of early NMO lesions is the loss of AQP4 immunoreactivity (Misu et al., 2007). In parallel, loss of GFAP immunoreactivity is described, accompanied by prominent perivascular deposits of immunoglobulin and complement components in a characteristic rim and rosette pattern (Fig. 1.2) (Lucchinetti et al., 2002; Misu et al., 2006; Roemer et al., 2007). These findings underpin the role of astrocytes as the primary target in NMO. In acute lesions, the inflammatory infiltrate consists of polymorphnuclear cells (PMNs) and eosinophilic granuloctyes, monocytes and $T$ cells. At later time points, macrophages are the dominant leukocyte population (Lucchinetti et al., 2002). Vessel walls become thickened and hyalinised (Lefkowitz and Angelo, 1984; Lucchinetti et al., 2002; Mandler et al., 1993). In addition to demyelination (DM) and oligodendrocyte apoptosis, cavitation, necrosis, and axonal loss (Fig. 1.2) were described in grey and white matter lesions in the spinal cord (SC) and optic nerve (ON) (Mandler et al., 1993; Parratt and Prineas, 2010). Less destructive NMO lesions have been found in the SC and medullary tegmentum with inflammation and loss of AQP4 immunoreactivity, but no DM and tissue necrosis, suggesting that a potential reversible NMO lesion type may exist (Roemer et al., 2007).
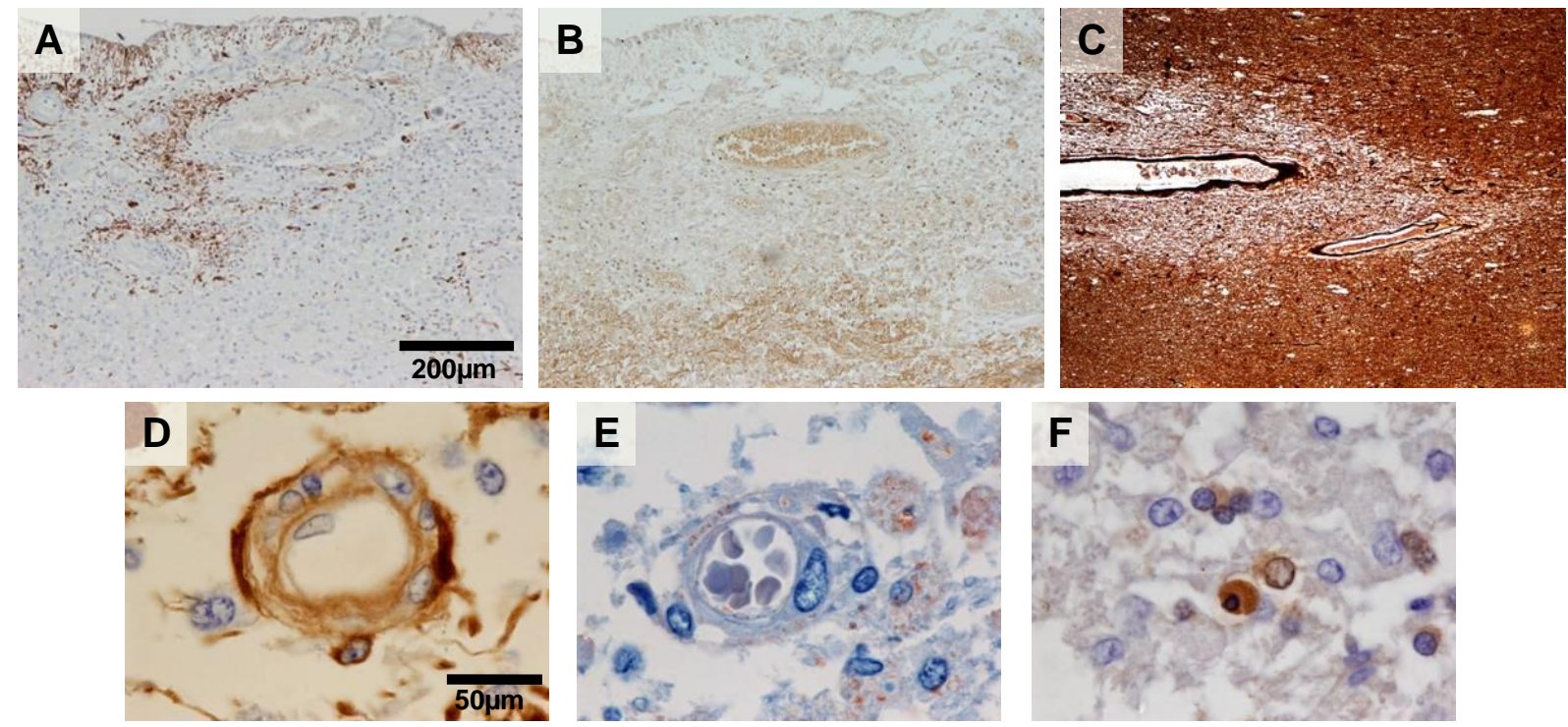

Figure 1.2: NMO lesion pathology in a 77 year old woman seropositive for anti-AQP4 antibodies. Lesions show extensive loss of astrocytes in GFAP staining (A), accompanied by severe DM (B; MBP). Severe axonal loss is one of the hallmarks of NMO (C; Bielschowsky silver impregnation). Additionally, lesions are characterized by lg depositions around the blood vessels (D, anti-lgM), complement deposits (E; anti-C9) and apoptotic oligodendrocytes (F; P25 staining). 


\subsubsection{Aquaporin 4}

Aquaporins are membrane proteins which regulate the flow of water in cells. Among the thirteen known aquaporins in mammals, aquaporin 4 (AQP4) is the main regulatory channel for water homeostasis in the CNS (Amiry-Moghaddam and Ottersen, 2003). Two isoforms exist in humans and rodents, namely M1 and M23 (Fig. 1.3) (Moe et al., 2008; Rash et al., 2004). AQP4 (mainly M23) is expressed in a polarized fashion in astrocyte membranes apposed to blood vessels, where it covers $30-40 \%$ of the astrocytic membrane (Wolburg, 1995). Besides astrocyte end-feet, AQP4 is also expressed in the nervous system in ependymal cells of the ventricles, meningeal cells of the pia mater, in astrocytes of the posterior optic nerve and in cells of the inner ear (Graber et al., 2008). Outside the nervous system, AQP4 immunoreactivity can be found in the kidney, respiratory tract, digestive tract, skeletal muscles and integumentary (Ma et al., 1997).

Most of the functions of AQP4 in the CNS are deduced from animal models deficient for AQP4. AQP4 knockout mice were generated in 1997 by targeted gene disruption and showed normal brain structure, vasculature and BBB function (Ma et al., 1997). Challenged by water intoxication AQP4 mice had a significantly improved survival and reduced swelling in astrocytic foot processes compared to controls (Manley et al., 2000). A significant reduction in brain swelling was also observed after middle cerebral artery occlusion (Manley et al., 2000). Besides the described function of AQP4 in brain edema, AQP4 is also involved in the migration of astrocytes. The localized swelling of lamellipodia is dependent on water flux across the plasma membrane and is important for cell migration. AQP4 deficient astrocytes migrate slower and show fewer lamellipodia (Saadoun et al., 2005). Finally, AQP4 deficiency results in a compromised clearance of extracellular $\mathrm{K}^{+}$and this impaired uptake might influence neural signal transduction (Padmawar et al., 2005). 
A

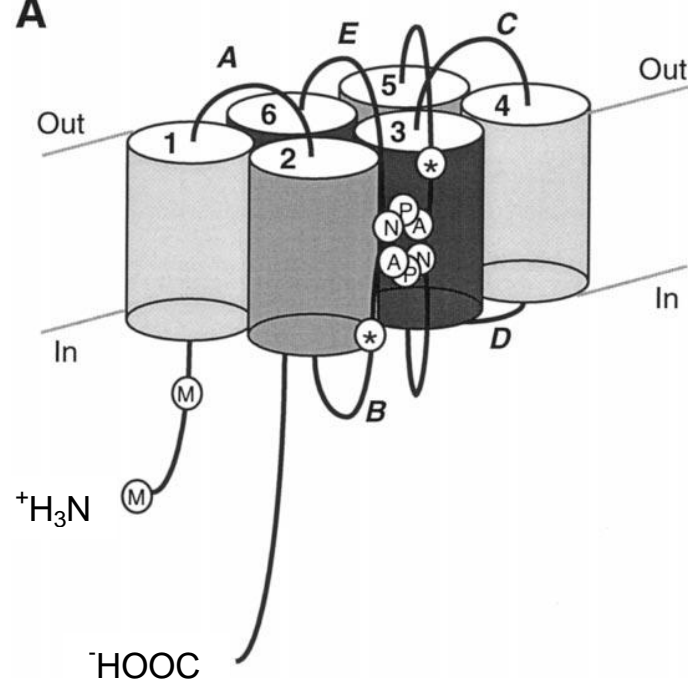

B

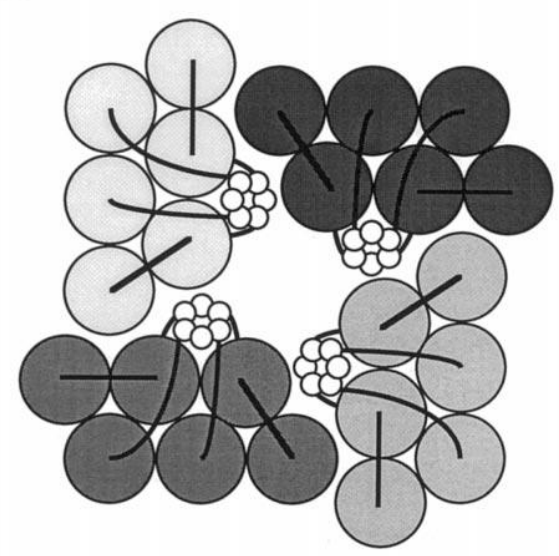

Figure 1.3: Model of AQP4. (A) 3-D structure shows the 6 domains and interconnecting loops and the two translation initiation sites M1 and M23 of AQP4. (B) Extracellular view showing the arrangement of AQP4 monomers into stable tetramers in the membrane. Adapted from Wells (1998).

\subsubsection{Effect of astrocytic overexpression of the fibroblast growth factor 9 (FGF9) on brain parenchymal cells}

\subsubsection{FGF9}

Fibroblast growth factors (FGFs) are a large family of polypeptide growth factors that are found in organisms ranging from nematodes to humans. In vertebrates, the 23 members of the FGF family range in molecular mass from 17 to $34 \mathrm{kDa}$ and share 13 $71 \%$ amino acid identity. They are involved in oligodendrogenesis, astrogenesis, embryonic development and wound healing. The two main receptors that FGFs bind to are FGFR (fibroblast growth factor receptor) 2 and FGFR3.

FGF9, first described as a glioma-derived growth factor (GDGF), is a heparin-binding growth factor protein containing 205 amino acid residues. In the adult CNS, FGF9 is known to be produced mostly by neurons and oligodendrocytes (Kanda et al., 1999; Nakamura et al., 1997; Todo et al., 1998), but also production and secretion by astrocytes has been described (Nakamura et al., 1999). Lin and coworkers (2009) showed that neuron-derived FGF9 is essential for migration of granule neurons in the cerebellum of mice. Additionally, FGF9 has been reported to have a neuroprotective effect on dopaminergic neurons (Huang and Chuang, 2010; Huang et al., 2009). 
Therefore, it is tempting to speculate that high FGF9 levels might be an endogenous repair or protective mechanism which might help to protect neurons.

\subsection{Effect of FGF9 on astrocytes}

On the one hand FGF9 is able to stimulate the proliferation and activation of glial cells and other cells that express FGF receptors. On the other hand in vitro experiments showed a strong inhibition of astrocytic differentiation and an almost total lack of GFAP positive astrocytes up to 7 days after treatment with FGF9. A total inhibition required prolonged treatment with FGF9, but for partial inhibition an $1 \mathrm{~h}$ pulse was sufficient (Lum et al., 2009b). Another study described the role of FGF9 in downregulation of astroglial gap junctions and functional coupling by decreasing the expression of the gap junction protein connexin 43 in CNS astroglial cultures (Reuss et al., 2000).

\subsection{Effect of FGF9 on oligodendrocytes and OPCs}

Previous studies investigating the effects of FGF9 in pure oligodendrocyte cultures have reported an inhibition of proteolipid protein (PLP) and myelin-basic protein (MBP) expression during oligodendrocyte differentiation (Cohen and Chandross, 2000) and an increase in process outgrowth in differentiated oligodendrocytes (Fortin et al., 2005). It has been reported that low FGF9 levels can lead to a modest effect on oligodendrocyte proliferation, whereas at higher concentrations, oligodendrocyte maturation was slowed down (Lum et al., 2009b).

\subsection{Effect of FGF9 on remyelination (RM)}

A low density microarray study investigating growth factors, cytokines and their receptors in MS lesions (developed and performed at the Max Planck Institute for Neuroimmunology by Hema Mohan and Edgar Meinl, unpublished data), revealed an association between the failure of RM and an upregulation of FGF9 in demyelinated compared to remyelinated lesions. FGFR3 expression was also upregulated in demyelinated lesions. 
Christopher Linington and colleagues (University of Glasgow, unpublished data) studied the effects of exogenous FGF9 on myelination in vitro. They found, that FGF9 inhibits the formation of myelin oligodendrocytes glycoprotein (MOG)-/PLP/MBP-positive myelin sheaths in a dose dependent manner associated with the selective suppression of MOG protein expressed by highly differentiated PLP-/MBPpositive oligodendrocytes. These cells can still contact and partially ensheath axons but fail to elaborate continuous compact myelin sheaths. The authors conclude that local expression of FGF9 may contribute directly to the failure of lesions to remyelinate.

\subsubsection{Aims}

Astrocytes are major players in both preventing and driving CNS diseases. In NMO, they are the targets of the immune response. The present thesis examines the pathogenesis of NMO-like focal lesions and studies the role of astrocyte-secreted FGF9 in experimental DM.

The aim of the first project is to develop experimental models of NMO and to study tissue damage and repair of NMO-like lesions.

The second project is based on recent findings that suggest a crucial role for FGF9 in RM. I used focal overexpression of FGF9 in astrocytes to study the role of this growth factor on astrocytes, oligodendrocytes and RM. 


\section{$2 \quad$ Materials and Methods}

For supplementary information on conventional procedures, solutions, chemicals, instruments, equipment and manufacturers, see Appendix A1, A2 and A3.

\section{$2.1 \quad$ Animals}

All in vivo experiments were carried out in adult (170-240g) female-inbred Lewis rats ( $n=244)$ purchased from Harlan Winkelmann $\mathrm{GmbH}$ (Borchen, Germany) or in adult C57BL/6 mice $(n=24)$ from Charles River (Sulzfeld, Germany). The animals were kept in cages of 6 animals each on a 12:12hrs light/dark cycle with food and water ad libitum. Newborn $\mathrm{P}_{0}$ Wistar rats were used as the source of oligodendroglial and astroglial cells. All experiments were accredited by the Bezirksregierung Braunschweig, Germany.

\section{$2.2 \quad$ NMO animal models}

To study the pathogenesis of NMO AQP4-specific bivalent recombinant human $\lg _{1}$ recombinant monoclonal antibodies ( $\mathrm{rAbs}$ ) were reconstructed from the paired heavy- and light-chain sequences of cerebrospinal fluid (CSF) plasma cell clones from an NMO patient after a first clinical attack (Bennett et al., 2009). This work was done in the laboratory of Jeffrey Bennett in the Department of Neurology and Ophthalmology, University of Colorado, Denver, USA. For the present study the recombinant anti-AQP4 antibody 53 (rAb-53) and a control-recombinant Ab 2B4 (ctrl$r A b)$ against the measles-virus-nucleocapsid protein were used. Furthermore, for EAE experiments, also modified variants of $\mathrm{rAb}-53$ with inserted point mutations were used. These antibodies were defective in complement activation, or in antibodydependent cell-mediated cytotoxicity (ADCC), or in both.

\subsubsection{Generation of focal NMO-like lesions in vivo}

To investigate the effects of the $\mathrm{rAb}-53$ in rats and to study the pathogenesis and repair in NMO-like lesions, a non-T-cell-dependent model was generated. 


\subsubsection{Intracerebral stereotactic injection}

Rats were intraperitoneally (i.p.) anaesthetized by injection of ketamine $(60 \mathrm{mg} / \mathrm{kg}$ bodyweight) and xylazine (8mg/kg bodyweight). After loss of consciousness, a rostrocaudal cut was performed to gain access to the skull. Next, the animal was mounted in a stereotactic device. A fine hole was drilled into the skull $1 \mathrm{~mm}$ caudal and $2 \mathrm{~mm}$ sagittal to the bregma, until only a thin layer of bone was left to avoid damage to the brain. The skull and the meninges were then carefully opened with a microdissecting knife. $1 \mu \mathrm{l}$ of $\mathrm{rAb}-53(2.5 \mathrm{mg} / \mathrm{ml})$ or ctrl-rAb $(2.5 \mathrm{mg} / \mathrm{ml})$ diluted in serum was injected stereotactically by a fine calibrated glass capillary into the M1 motorcortex region or corpus callosum. Furthermore, to mark the injection site, monastral blue was added to the antibody and serum mixture. The solution was administered very slowly during a period of 3 minutes to avoid tissue damage. After injection the capillary was carefully withdrawn and the skin was sutured. To provide analgesia, buprenorphine (Temgesic(C) was administered at $0.03 \mathrm{mg} / \mathrm{kg}$ during surgery, 6 and $12 \mathrm{hrs}$ later. Finally, the animals were perfused at various time points after injection and the tissues were processed for immunohistochemistry.

\subsubsection{Pharmacological experiments to determine the role of NMDA and $P_{2} X_{7}$ receptors in oligodendrocyte death}

The focal NMO model is characterized by astrocyte loss due to the lytic effect of rAb53 and complement followed by oligodendrocyte loss. To investigate the role of glutamate and ATP in oligodendrocyte death, NMDA and $\mathrm{P}_{2} \mathrm{X}_{7}$ receptor antagonists were applied $(n=3)$.

The NMDA receptor antagonists D-AP5 and MK-801 as well as the $\mathrm{P}_{2} \mathrm{X}_{7}$ receptor antagonists periodate oxidized adenosine triphosphate (OATP) and brilliant blue $G$ (BBG) were injected intracerebrally together with the rAb-53 and human complement (total volume: $2 \mu \mathrm{l}$ ). Concentration of the antagonists was $500 \mu \mathrm{mol} / \mathrm{L}$. Control animals received only rAb-53 and complement or only the receptor antagonists. After $24 \mathrm{hrs}$ the animals were perfused and tissue processed for histological assessment. 


\subsubsection{I.v. transfer of $\mathrm{rAb}-53$ into animals with experimental autoimmune encephalomyelitis (EAE)}

A "systemic" model of NMO was established to study the neuropathological features in Lewis rats after immunization with MBP-peptide.

\subsubsection{Sensitization procedure}

Female rats $(\mathrm{n}=36)$ were anaesthetized with isoflurane and immunized with $100 \mu \mathrm{g}$ guinea pig $\mathrm{MBP}_{72-85}$ (gp-MBP-peptide) emulsified in complete Freund's adjuvant containing $5 \mathrm{mg} / \mathrm{ml}$ inactivated Mycobacterium tuberculosis H37 Ra. After 7-10 days the animals showed the first clinical symptoms (see EAE score in Tab. 2.1).

Table 2.1: EAE score on scale 0 to 5

\begin{tabular}{|l|l|}
\hline score & clinical observation \\
\hline \hline 0 & no obvious changes in motor functions \\
\hline \hline 1 & limp tail \\
\hline \hline 2 & limp tail and weakness of hind legs \\
\hline \hline 3 & limp tail and complete paralysis of hind legs \\
\hline \hline 4 & limp tail, complete hind leg and partial front leg paralysis \\
\hline \hline 5 & complete hind and complete front leg paralysis \\
\hline
\end{tabular}

\subsubsection{Antibody application}

When the animals showed first signs of disease (scores 0.5 or 1 ), $500 \mu l$ of the recombinant antibodies $(5 \mathrm{mg} / \mathrm{ml})$ were administered into the retrobulbar venous plexus. After $30 \mathrm{hrs}$ the animals were perfused and brains, spinal cords and kidneys were prepared for histological assessment.

\subsubsection{Kidney function parameters}

Urine of MBP-primed rats $(n=8)$ was collected 30hrs after antibody application by gently squeezing the bladder. 
A volume of $100 \mu \mathrm{l}$ of the collected urine was centrifuged at $1000 \mathrm{rpm}$ for $5 \mathrm{~min}$ using a cytospin centrifuge. In this way, the cell debris and protein were separated onto glass slides from the aqueous part of the urine and then stained according to Pappenheim.

The protein concentration in the urine was determined using commercial dip sticks, which change their color depending on the content of protein.

\subsubsection{The effect of rAb-53 in vitro}

The in vivo experiments $(n=3)$ were complemented by in vitro studies examining the effects of $\mathrm{rAb}-53$ on oligodendrocyte, astrocyte, and mixed glial cultures.

\subsubsection{Isolation of primary glial cells from newborn animals}

As a source of primary glial cells, heads of newborn rats $\left(P_{0}-P_{1}\right)$ were cut and transferred in fresh Hank's buffered salt solution (HBSS) medium in a Petri dish to rinse off the blood. The heads were opened with fine scissors, and the brains removed with forceps and rinsed in fresh HBSS. The cerebella were removed and discarded. The meninges were removed with fine forceps to prevent that meningeal fibroblasts interfere with the glial cell growth. Afterwards the brains were placed in $0.25 \%$ Trypsin-EDTA solution in $37^{\circ} \mathrm{C}$ to dissociate the tissue and release the cells. After washing with HBSS the brains were transferred into basal medium eagle (BME). A cell suspension was generated by trituration of the dissected brains in BME medium with a $10 \mathrm{ml}$ glass pipette. The suspension was seeded into $75 \mathrm{~cm}^{3}$ poly-Llysin (PLL)-coated cell culture flasks containing $10 \mathrm{ml}$ BME medium. The cells, including astrocytes, oligodendrocytes and microglia, were grown for 10 to 14 days in an incubator $\left(37^{\circ} \mathrm{C}\right)$. Medium was changed every 2 to 3 days.

\subsubsection{Culture of extracted cells}

In order to harvest oligodendrocytes and astrocytes from the mixed glial cell population, microglia cells were first shaken off by tapping the flask against the desk while controlling the process microscopically. Afterwards, cells were diluted twice with BME medium and fresh BME medium was added to the cell culture flasks. To 
collect the oligodendrocytes, flasks were vigorously shaken horizontally 20 times by hand. The medium containing the detached cells was transferred into a falcon tube through a $40 \mu \mathrm{m}$ cell strainer to separate the larger cells from the oligodendrocytes. Then cells were centrifuged using an Eppendorf centrifuge at $800 \mathrm{rpm}$ for $10 \mathrm{~min}$. The supernatant was discarded and the cell pellet was resuspended in $2 \mathrm{ml}$ Super-Sato medium. To remove remaining microglia, the cell suspension was transferred to a $3.5 \mathrm{~cm}$-diameter Petri dish, where the microglia were allowed to settle for $30 \mathrm{sec}$. The cell suspension was then transferred into a falcon tube and the cells were counted using a Neubauer counting chamber. About 15,000 cells were plated on each PLLcoated, $\mathrm{HNO}_{3}$-treated cover slip. These cover slips had already been placed in a 24(or 12-) well plate and incubated with Super-Sato medium the day before. The cells were incubated at $37^{\circ} \mathrm{C}$ and $5 \% \mathrm{CO}_{2}$ for $24 \mathrm{hrs}$ before starting the experiments.

To collect the astrocytes, the nearly microglia/oligodendrocyte-free cell culture flasks were washed with serum-free medium and then treated with $0.05 \%$ trypsin-EDTA for $5 \mathrm{~min}$ at room temperature while being automatically shaken to detach astrocytes from the flask surface. The reaction was stopped with BME medium. The supernatant was collected and centrifuged for $10 \mathrm{~min}$ with $800 \mathrm{rpm}$. The cell pellet was resuspended in $2 \mathrm{ml}$ Super-Sato medium and the astrocytes were counted using a Neubauer counting chamber. About 100,000 cells were plated on one PLL-coated, $\mathrm{HNO}_{3}$-treated cover slip. Astrocytes were incubated, just like the oligodendrocytes, for $24 \mathrm{hrs}$ before starting the experiments.

\subsubsection{Pharmacological experiments to determine the role of NMDA and $P_{2} X_{7}$ receptors for oligodendrocyte death in vitro}

When the astrocytes had settled, the cells were incubated with rAb-53 $(1 \mu \mathrm{g} / 200 \mu \mathrm{l})$ and human serum. Control wells received a) no antibodies b) serum or c) antibody alone. To determine, if the NMDA and $\mathrm{P}_{2} \mathrm{X}_{7}$ receptor antagonists influence the extent of oligodendrocyte death, some wells were incubated with rAb-53, human serum and one of the antagonists (conc: $50 \mu \mathrm{M}$ ). After $24 \mathrm{hrs}$, the supernatants were collected and cell viability and cell cytotoxicity assays were performed. Afterwards, astrocyte cultures were fixed using 4\% PFA and stained for DAPI, GFAP and O4.

Supernatants of astrocytes incubated with $\mathrm{rAb}-53$ and human serum were added to primary oligodendrocyte cultures for $24 \mathrm{hrs}$. To determine if NMDA and $\mathrm{P}_{2} \mathrm{X}_{7}$ receptor 
antagonists prevent oligodendrocyte death, some wells were treated with a NMDA or $\mathrm{P}_{2} \mathrm{X}_{7}$ antagonist. Control wells were left untreated, received serum or antibody alone. After the incubation time, supernatants were collected to perform a cell cytotoxicity assay, and cell viability was determined. Cells were fixed and stainings for DAPI, MBP and O4 were performed.

\subsection{Cell viability assay (WST-1)}

Cell viability and the rate of cell proliferation were measured by the colorimetric WST1 (water soluble tetrazolium) assay. The principle of this assay is based on the ability of dividing cells to reduce tetrazolium salt to soluble formazan by mitochondrial dehydrogenases (Fig. 2.1).

$250 \mu$ l of ready-to-use WST-1 solution was added to each well (24-well plate) and incubated for $10 \mathrm{~min}$ (astrocytes) or $20 \mathrm{~min}$ (oligodendrocytes) at $37^{\circ} \mathrm{C}$. Subsequently, $100 \mu \mathrm{l}$ of each supernatant was transferred in a 96-well plate and the absorbance was measured by an ELISA reader at 450 and $655 \mathrm{~nm}$.

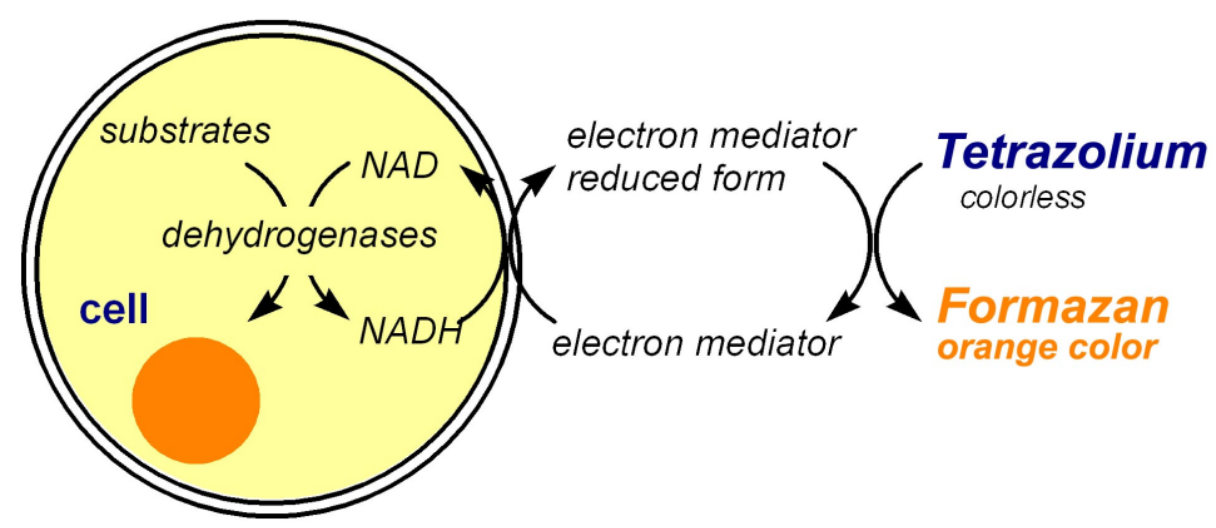

Figure 2.1: Principle of the WST-1 assay. WST-1 is reduced from a colorless tetrazolium to a detectable orange formazan by dehydrogenases of viable cells using NAD an electron mediator. http://www.dojindo.com/newimages/principal-SK.jpg

\subsection{CytotoxOne membrane integrity assay}

The CytotoxOne homogeneous membrane integrity assay measures the concentration of LDH (lactate dehydrogenase) which is released to the medium by damaged cells. LDH converts lactate to pyruvate. In this assay, this principle is 
coupled to diaphorase, which converts non-fluorescent resazurin to fluorescent resorufin (Fig. 2.2).

$100 \mu \mathrm{l}$ of each supernatant was transferred into a 96-well plate and the temperature was equilibrated to $22^{\circ} \mathrm{C}$. Then $100 \mu \mathrm{l}$ of the CytotoxOne reagent was added to each well, mixed for $30 \mathrm{sec}$ and incubated for $10 \mathrm{~min}$ at $22^{\circ} \mathrm{C}$. To end the reaction, $50 \mu \mathrm{l}$ of a stop solution was added. The degree of fluorescence was measured by a Tecan Safire plate reader at 560nm excitation and 590nm emission.

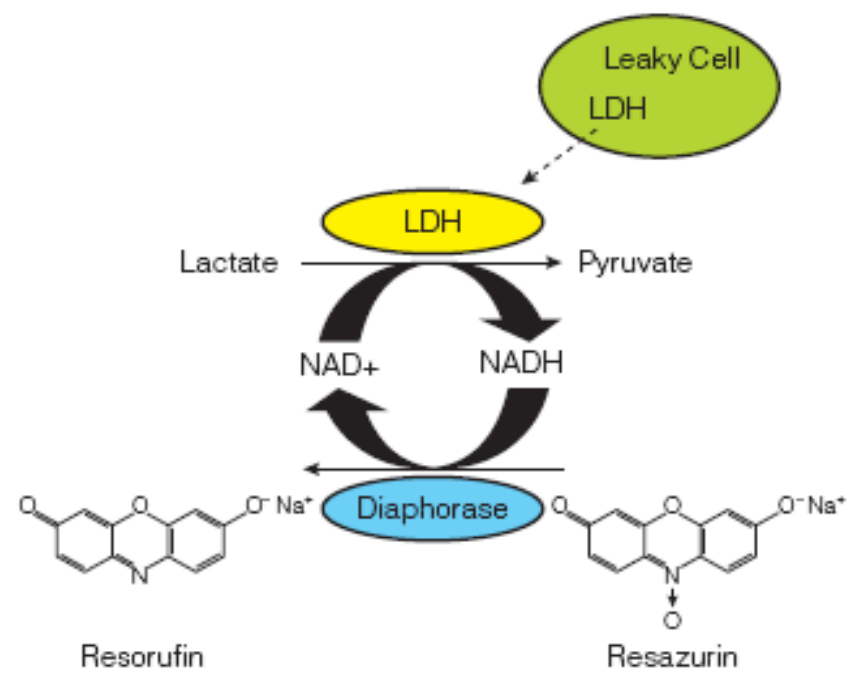

Figure 2.2: Principle of CytotoxOne membrane integrity assay. The assay measures the release of LDH by damaged cells using a coupled enzymatic principle that results in the conversion of resazurin into the fluorescent compound resofurin. www.promega.com/tbs/tb306/tb306.html

\subsection{Overexpression of FGF9 in demyelinating animal models}

\subsection{1}

\section{Adeno-associated viral 6 (AAV-6)-based vector as a tool to express FGF9 in astrocytes}

To investigate the influence of FGF9 on astrocytes, oligoendrocytes and the process of $\mathrm{RM}$, a replication-deficient vector based on adeno-associated virus 6 (AAV-6) was used to overexpress this protein in astrocytes.

The AAV-6 vectors were constructed by Dr. Sebastian Kügler, Dept. of Neurology, UMG) (Kugler et al., 2003). The genome of the control viral vectors consisted of the astrocyte-specific GFAP promotor and the CDNA for enhanced green fluorescent protein (EGFP). Note that these EGFP-AAV-6 constructs contain a woodchuck hepatitis post-transcriptional control element (WPRE) which stabilizes mRNA, resulting in a 2-10 fold higher rate of protein expression. The constructed vectors 
were propagated in 293 cells using a helper plasmid (Grimm et al., 2003) and purified (Malik et al., 2005). After dialysis, genome titres were determined, and purification and identification of infectious titres were performed (Kugler et al., 2003) (Fig. 2.3).

To insert the FGF9 CDNA into the AAV-6-based vector, Dr. Andreas Junker, Institute for Neuropathology, UMG, slightly modified the construct. The EGFP insert was cut out and replaced by the FGF9 cDNA, which was derived from a commercial topo vector (Fig. 2.4).

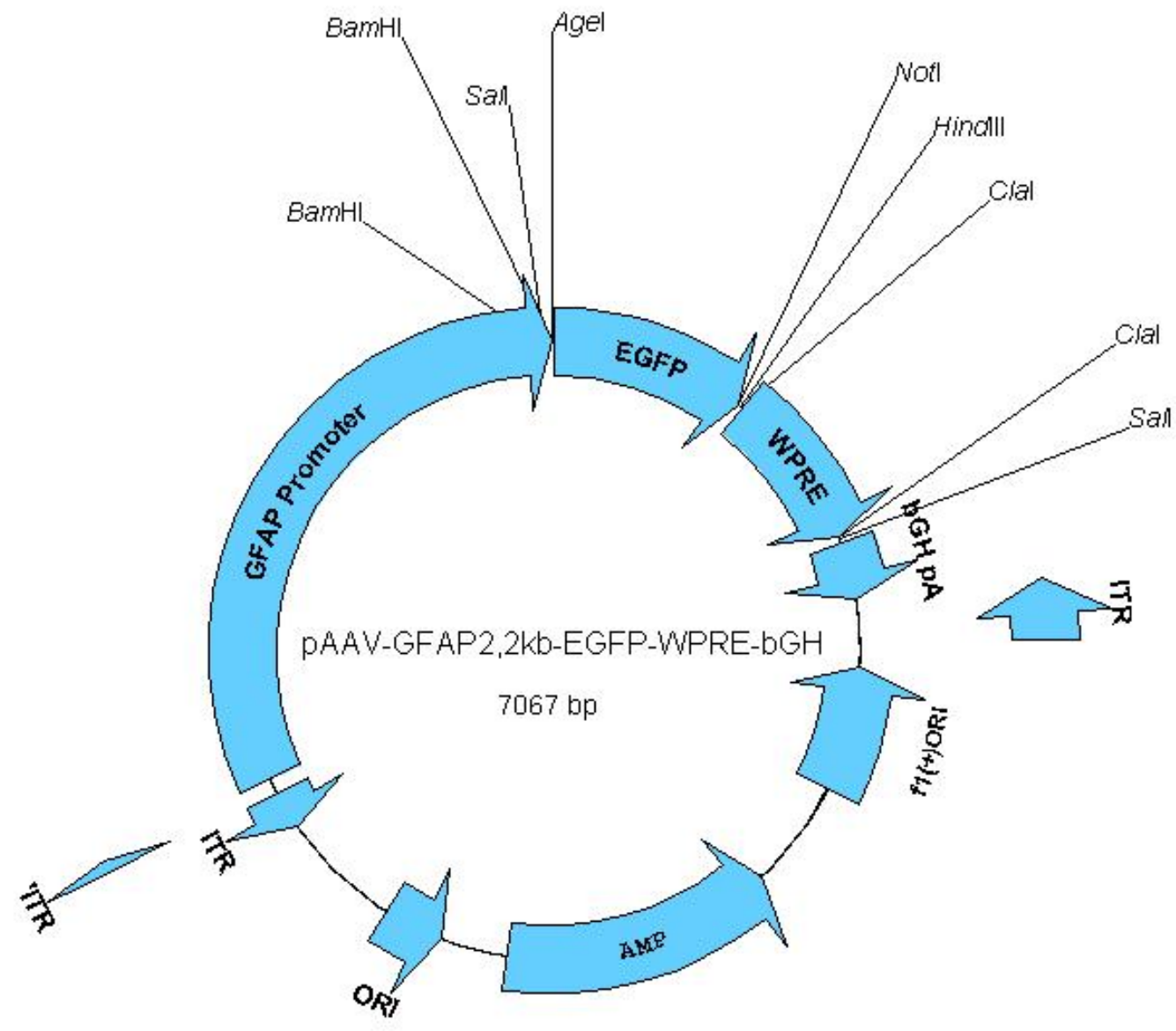

Figure 2.3: Vector card of the control AAV-6 with EGFP insertion constructed by Sebastian Kügler. 


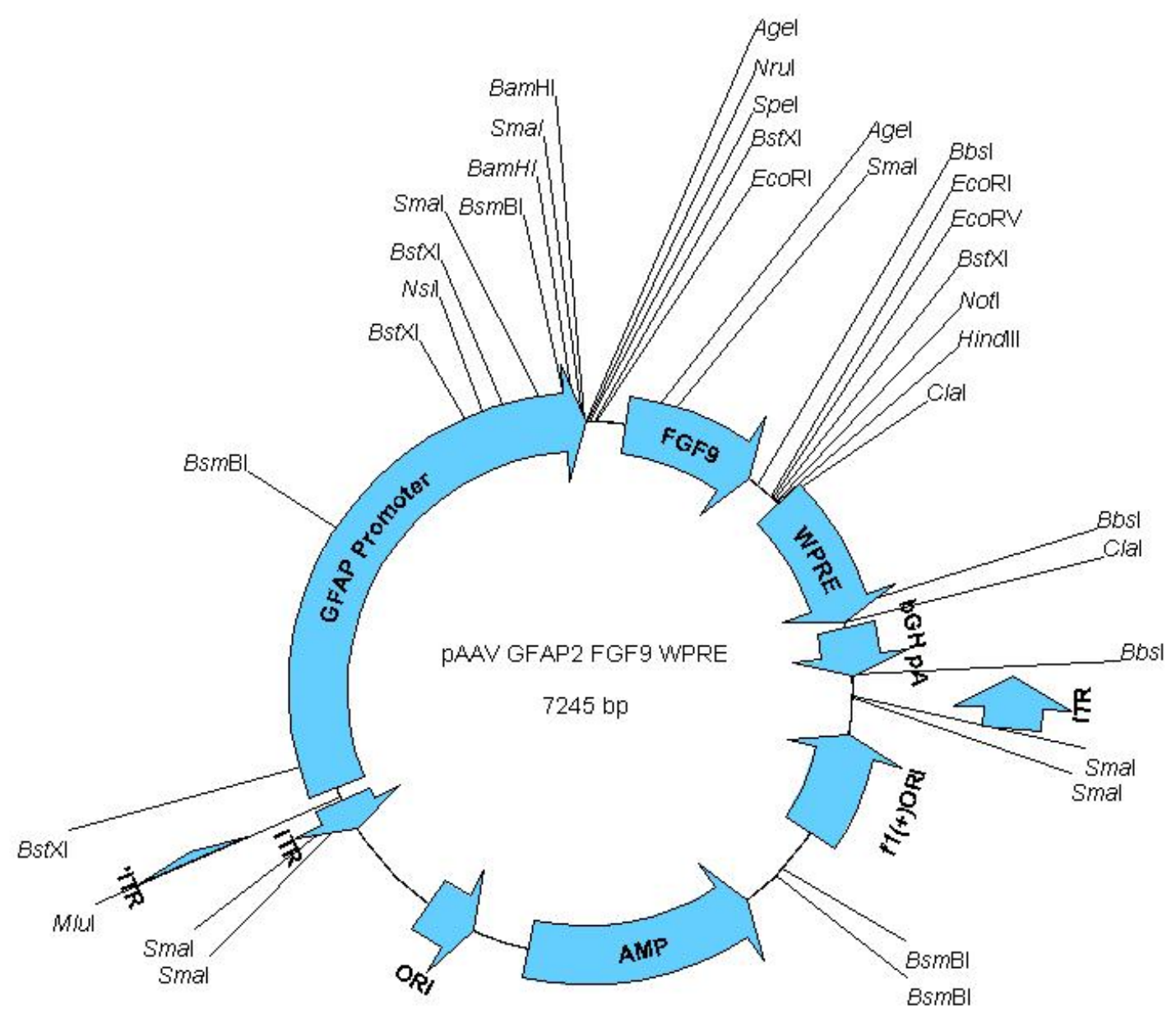

Figure 2.4: Vector card of the FGF9 containing AAV-6 modified by Andreas Junker.

\subsubsection{Injection of viral vectors in focal EAE}

To determine if FGF9 influences DM or RM, focal experimental autoimmune encephalomyelitis (fEAE) was performed (Merkler et al., 2006) and FGF9 or EGFP expression was induced by an AAV-6-based vector.

In contrast to EAE models which generally affect the spinal cord and rarely the brain, fEAE allows one to assign a demyelinating lesion to a specific region of the brain.

Adult Lewis rats $(n=39)$ were immunized at the base of the tail with a subclinical dose $(50 \mu \mathrm{g})$ of recombinant rat $\mathrm{MOG}_{1-125}$ (rrMOG) protein emulsified in incomplete Freund's adjuvant. This immunization induces anti-MOG-antibody production after 18-24 days without clinical symptoms except slight weight loss. 


\subsection{Enzyme-linked immunosorbent assay (ELISA)}

The anti-MOG antibody titer in serum samples of MOG-immunized rats was determined by ELISA. Blood samples of rats were collected from the tail vein 3-4 weeks after immunization. Serum was separated from other blood components by centrifugation for $15 \mathrm{~min}$ at $4^{\circ} \mathrm{C}$ and $1000 \mathrm{rpm}$. 96 well plates were coated with $0.8 \mu \mathrm{g}$ rrMOG $_{1-125}$ diluted in PBS containing $0.05 \%$ Tween per well and incubated for $4 \mathrm{hrs}$. Afterwards, the plates were washed 6 times with PBS/Tween $(0.05 \%)$ and blocked with $2 \%$ BSA diluted in PBS for $2 \mathrm{hrs}$ at $37^{\circ} \mathrm{C}$. The prepared plates were washed with distilled water and different dilutions of the serum samples were added to the wells and incubated for $2 \mathrm{hrs}$ at room temperature. The plates were washed again using PBS/Tween solution and incubated for $2 \mathrm{hrs}$ with an HRP coupled anti-rat Ig antibody. After another washing step, $100 \mu \mathrm{l}$ of TMB substrate was added to the wells. After approximately $15 \mathrm{~min}, 50 \mu \mathrm{l}$ of $1 \mathrm{~N} \mathrm{H}_{2} \mathrm{SO}_{4}$ stop solution was added to the samples and the absorbance was measured by an ELISA reader at $450 \mathrm{~nm}$ and $540 \mathrm{~nm}$. The animals with the highest serum antibody concentrations were used for intracerebral viral vector injection.

\subsection{Induction of focal EAE lesions and intracerebral viral vector injection}

To induce a demyelinating lesion in the cortex or corpus callosum, the cytokines IFN-y and TNF- $\alpha$ were injected stereotactically $1 \mathrm{~mm}$ caudal and $2 \mathrm{~mm}$ sagittal to the bregma (for further details see 2.2.1.1). Maximal DM is achieved after 3 days. The lesions then almost fully remyelinate within 14 days. To study the influence of FGF9 on DM and RM, cytokines were injected $(2 \mu \mathrm{l})$ together with the FGF9-expressing viral vector. Controls were injected with a viral vector expressing EGFP together with cytokines or PBS with cytokines. The animals were perfused 3 and 10 days after injection.

\subsubsection{Injection of viral vectors in the cuprizone model}

Using a further demyelinating model to assess the influence of FGF9 on DM and RM, the toxic copper chelator cuprizone was used in mice $(n=24)$. Cuprizone induces DM 
in the cortex and corpus callosum. Mice were fed with a cuprizone $(0.25 \%)$ diet for 5 weeks. After 4 weeks mice were stereotactically injected $(2 \mu l)$ with FGF9-AAV-6based vector, EGFP-control-AAV-6-based vector or PBS into the mouse brain (for further details see 2.2.1.1.). The animals were perfused 7 and 10 days post injection and brains were processed for histological assessment.

\section{$2.4 \quad$ Histology}

At the end of the experiments, the animals were anaesthetized by i.p. injection of a lethal dose of $14 \%$ chloral hydrate. After loss of nociceptive and eye lid reflexes, transcardial perfusion was performed through the left heart ventricle with PBS followed by $4 \%$ paraformaldehyde (PFA). Brains, SC, livers and spleens were collected and stored for $48 \mathrm{hrs}$ at $4^{\circ} \mathrm{C}$ in $4 \%$ PFA. After a washing step with PBS, tissue was dissected into 4-6mm-thick pieces and washed in water. For embedding in paraffin, the tissues were gradually dehydrated by performing graded alcohol/xylene/paraffin series using an automated tissue processor overnight. The tissues were then embedded in paraffin blocks, cut into thin $1 \mu \mathrm{m}$ sections using a sliding microtome and mounted on glass slides. For tissue previously injected intracerebrally, the injection site was identified by traces of monastral blue.

Prior to the staining procedures, sections were deparaffinized and hydrated by performing graded xylene and isopropyl alcohol steps. To do so, they were immersed 4 times in xylene $(5 \mathrm{~min})$, once in isoxylene $(1 \mathrm{~min})$, twice in $100 \%$ isopropyl alcohol (4min), once in $90 \%, 70 \%$ and $50 \%$ isopropyl alcohol (3min) and washed with distilled water. Stained sections were dehydrated after the staining procedures by performing the above described series in reversed order and mounted in DePex medium.

\subsubsection{Histochemical stainings}

Hematoxylin and eosin (H\&E) staining is used for a general overview and evaluation of tissue especially with regard to the morphology of inflammatory, infiltrating and apoptotic cells. Hematoxylin stains cell nuclei blue by binding to basic nucleoproteins. 
In contrast, eosin dyes acidophilic and basic extra- and intracellular proteins pink and red (eosinophilic).

Sections were washed 3 times with distilled water and incubated for 8min in Mayer's hemalaun, then shortly washed with water, differentiated by dipping approx. $1 \mathrm{~min}$ in $1 \% \mathrm{HCl}$ alcohol and blued for $10 \mathrm{~min}$ under running tap water. Afterwards, slides were placed into $1 \%$ eosin solution for $6 \mathrm{~min}$, washed with water, dehydrated and mounted.

Luxol fast blue/periodic acid-Schiff (LFB/PAS) staining highlights demyelinated areas in CNS tissue by dyeing lipoproteins of myelin deep blue via LFB, and nonmyelinated/demyelinated parenchyma pink via PAS. After the $90 \%$ isopropyl alcohol step, the sections were placed in LFB solution in $60^{\circ} \mathrm{C}$ overnight and afterwards washed with $90 \%$ isopropyl alcohol. For differentiation, $0.05 \%$ lithium carbonate in $\mathrm{H}_{2} \mathrm{O}$ was used followed by $70 \%$ isopropyl alcohol. Distilled water was applied to stop this washing step until only myelin was stained deep blue. To start the PAS reaction, sections were put in $1 \%$ periodic acid for $5 \mathrm{~min}$, followed by washing for $5 \mathrm{~min}$ under running tap water, 5 min with distilled $\mathrm{H}_{2} \mathrm{O}$ and incubated in Schiff's solution for $20 \mathrm{~min}$. After this step, sections were washed again under running tap water. The hemalaun procedure, as described above, was performed to stain cell nuclei and the sections were then mounted.

Bielschowsky silver impregnation was performed to evaluate axonal integrity and loss. Because of the argyrophilicity of axons, insoluble silver nitrate is able to bind, causing a reduction in elementary silver and a black impregnation. The parenchyma is stained yellow to brown.

First sections were incubated in $20 \%$ silver nitrate $\left(\mathrm{AgNO}_{3}\right)$ solution in distilled $\mathrm{H}_{2} \mathrm{O}$ for $20 \mathrm{~min}$ in the dark and afterwards washed in distilled $\mathrm{H}_{2} \mathrm{O}$. $32 \%$ ammonium hydroxide in distilled $\mathrm{H}_{2} \mathrm{O}$ was added drop by drop to the silver nitrate solution until all precipitations cleared up while shaking. The sections were placed for $15 \mathrm{~min}$ in this cleared solution in the dark and washed with distilled $\mathrm{H}_{2} \mathrm{O}$ containing a few drops of ammonium hydroxide. About 10 drops of developer solution were added to the silver nitrate/ammonium hydroxide solution and the sections were developed for 5-6min until axons were stained black. Finally, the tissues were washed with distilled water and fixed with $2 \%$ sodium thiosulfate for $2 \mathrm{~min}$, dehydrated and mounted. 
Pappenheim is a common panoptic method for the staining of smears of air dried cytological materials like urine sediment. It dyes nuclei purple, cytoplasm of lymphocytes blue, monocytes grayish blue, erythrocytes reddish/brownish and granulocytes dark purple/red violet.

Glass slides containing cell debris from cytospins were air dried for 10min and incubated in May-Grünwald solution for 3min. They were then washed in PBS for $1 \mathrm{~min}$ and again incubated in Giemsa solution for $15 \mathrm{~min}$. Afterward slides were washed in PBS and distilled water for $1 \mathrm{~min}$, dehydrated and mounted with DePex.

\subsubsection{Immunohistochemistry (IHC)}

For optimal preservation of the morphology, tissues were fixed in $4 \%$ paraformaldehyde (PFA). However this causes a modification of tissue proteins and a loss of epitopes. In these cases paratopes of an antibody are not able to react with the antigens and a so-called epitope retrieval is needed.

Epitopes were demasked by heating the slides in $10 \mathrm{mM}$ citric acid, $1 \mathrm{mM}$ Tris-EDTA or Tris-HCL solution in a microwave for up to $5 \times 5$ minutes, refilling after each cycle with distilled water. After this procedure the slides had to cool down and were then washed with distilled water before performing immunohistochemical stainings.

Immunhistochemical stainings were performed to detect specific marker proteins for astrocytes, myelin-related proteins, infiltration/inflammation, axon pathology, cell density of mature oligodendrocytes and OPCs.

The antibodies applied were generated in rabbits, mice, or rats. Avidin-coupled peroxidase and 3,3'-diaminobenzidine tetrachloride (DAB) were used to visualize antibody binding of biotin-conjugated secondary antibodies. To visualize targets detected with the alkaline phosphatase anti-alkaline phosphatase (APAAP) method, Fast Red or Fast Blue were utilized as chromogens.

The slides were washed first in PBS, followed by incubation in $3 \% \mathrm{H}_{2} \mathrm{O}_{2}$ at $4^{\circ} \mathrm{C}$ for $20 \mathrm{~min}$ to block endogenous peroxidase. After being washed 3 times in PBS, the sections were blocked with $10 \%$ FCS in PBS at RT to inhibit unspecific antibody binding. The slides were incubated overnight with the specific primary antibody (see list of primary antibodies in Tab. 2.2) diluted in $10 \%$ FCS in PBS at $4^{\circ} \mathrm{C}$ in a wet chamber. The next day sections were washed 3 times with PBS and incubated with a 
biotinylated secondary antibody diluted in 10\% FCS in PBS, which binds specifically to the $\mathrm{Fc}$ region of the primary antibody. After $1 \mathrm{~h}$ the unbound antibody was removed by washing with PBS and the slides were placed for $1 \mathrm{~h}$ in $0.1 \%$ streptavidinhorseradish peroxidase (POX) diluted in 10\% FCS in PBS at RT in a humid chamber. Then the unbound peroxidase was removed by washing with PBS and the reaction was developed using DAB which was oxidized by the bound POX, generating a darkbrown staining. Sections were developed under microscopic control. To visualize cell nuclei, the hemalaun procedure, as described above, was performed and the sections were mounted.

Table 2.2: List of primary antibodies

\begin{tabular}{|l|l|l|l|l|}
\hline Antigen & Marker for & $\begin{array}{l}\text { Species/ } \\
\text { clone }\end{array}$ & Dilution & Manufacturer \\
\hline \hline APP & $\begin{array}{l}\text { amyloid precursor protein; } \\
\text { early axonal damage }\end{array}$ & $\begin{array}{l}\text { mouse/ } \\
22 \mathrm{C} 11\end{array}$ & $1: 3000$ & Chemicon \\
\hline \hline AQP4 & $\begin{array}{l}\text { water channel expressed on } \\
\text { astrocyte foot processes }\end{array}$ & rabbit & $1: 500$ & Millipore \\
\hline \hline C9 & complement factor 9 & $\begin{array}{l}\text { mouse/ } \\
\text { B7 }\end{array}$ & $1: 50$ & $\begin{array}{l}\text { kindly provided } \\
\text { by B.P. Morgan, } \\
\text { Cardiff, UK }\end{array}$ \\
\hline \hline $\begin{array}{l}\text { Caspase- } \\
3\end{array}$ & activated caspase-3 & $\begin{array}{l}\text { rabbit/ } \\
\text { C92-605 }\end{array}$ & $1: 500$ & BD-Pharmingen \\
\hline \hline CD3 & T-lymphocytes & $\begin{array}{l}\text { rat/ CD3- } \\
12\end{array}$ & $1: 200$ & Serotec \\
\hline \hline CNP & myelin forming cells & $\begin{array}{l}\text { mouse/ } \\
\text { SMI-91 }\end{array}$ & $1: 200$ & Covance \\
\hline $\begin{array}{l}\text { EAAT2 } \\
\text { (GLT-1) }\end{array}$ & $\begin{array}{l}\text { glutamate } \\
\text { expressed in astrocyte } \\
\text { membrane }\end{array}$ & $\begin{array}{l}\text { mouse/ } \\
1 \text { H8 }\end{array}$ & $1: 50$ & Novocastra \\
\hline \hline $\begin{array}{l}\text { CD68 } \\
\text { (ED1) }\end{array}$ & $\begin{array}{l}\text { macrophages/activated } \\
\text { microglia in rat }\end{array}$ & $\begin{array}{l}\text { mouse/ } \\
\text { ED1 }\end{array}$ & $1: 500$ & Serotec \\
\hline \hline FGF9 & fibroblast growth factor 9 & $\begin{array}{l}\text { mouse/ } \\
(\mathrm{D}-8)\end{array}$ & $1: 100$ & Santa Cruz \\
\hline
\end{tabular}


Materials and Methods

\begin{tabular}{|c|c|c|c|c|}
\hline GFAP & $\begin{array}{l}\text { glial fibrillary acidic protein; } \\
\text { astrocyte specific protein }\end{array}$ & rabbit & $1: 1000$ & Dako \\
\hline EGFP & $\begin{array}{l}\text { enhanced green fluorescent } \\
\text { protein }\end{array}$ & $\begin{array}{l}\text { mouse/ } \\
\text { 6AT316 }\end{array}$ & $1: 500$ & Abcam \\
\hline $\lg G$ & immunglobulin G & mouse & $1: 100$ & BioGenex \\
\hline $\lg G$ & immunglobulin G & rabbit & $1: 100$ & BioGenex \\
\hline Ki67 & proliferation marker & $\begin{array}{l}\text { mouse/ } \\
\text { Mib-1 }\end{array}$ & $18: 100$ & Dako \\
\hline Mac3 & $\begin{array}{l}\text { macrophages/activated } \\
\text { microglia in mice }\end{array}$ & $\begin{array}{l}\text { rat/ } \\
\text { M3/84 }\end{array}$ & $1: 200$ & Becton Dickinson \\
\hline MBP & myelin-basic protein & rabbit & $1: 1000$ & Dako \\
\hline NogoA & mature oligodendrocytes & $\begin{array}{l}\text { mouse/ } \\
11 C 7\end{array}$ & $1: 20000$ & $\begin{array}{l}\text { Kind gift from M. } \\
\text { Schwab, Zürich } \\
\text { (Oertle et al., } \\
\text { 2003) }\end{array}$ \\
\hline O4 & $\begin{array}{lcr}\text { expressed in most } \\
\text { maturation stages } & \text { of } \\
\text { oligodendrocytes } & \end{array}$ & $\begin{array}{l}\text { mouse/ } \\
\text { O4 }\end{array}$ & $1: 100$ & $R \& D$ \\
\hline Olig2 & $\begin{array}{l}\text { oligodendrocyte precursor } \\
\text { cells and mature } \\
\text { oligodendrocytes } \\
\text { (transcription factor) }\end{array}$ & rabbit & $1: 300$ & 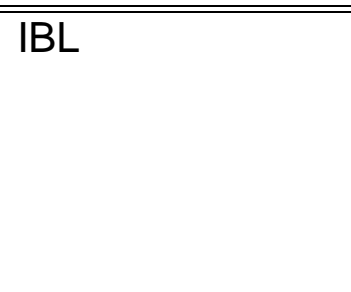 \\
\hline PLP & proteolipid protein & $\begin{array}{l}\text { mouse/ } \\
\text { plpc1 }\end{array}$ & $1: 250$ & Biozol \\
\hline S100B & astrocyte specific protein & rabbit & $1: 1000$ & Abcam \\
\hline
\end{tabular}

\subsubsection{Morphometry}

Cell densities were evaluated at a 400x magnification using an ocular counting grid. The extent of astrocyte loss was assessed using GFAP immunocytochemistry. GFAP-immunostained spinal cord or brain sections were scanned using a light microscope equipped with a digital camera. At least 15 spinal SC sections were examined per animal. Areas of complete astrocyte loss were measured using 
Analysis $^{\mathrm{TM}}$ software (University of Wisconsin, Madison, WI). The total area of astrocyte loss between groups was evaluated by analysis of variance (ANOVA).

\subsubsection{GFAP/NogoA/Olig2 triple immunofluorescence}

The marker for OPCs, Olig 2, detects immature oligodendrocytes, but also binds to NogoA-positive mature oligodendrocytes, though, giving a weaker nuclear staining signal (Kuhlmann et al., 2008). To distinguish both cell types and count densities of OPCs in astrocyte-depleted areas, triple immunofluorescence stainings were performed.
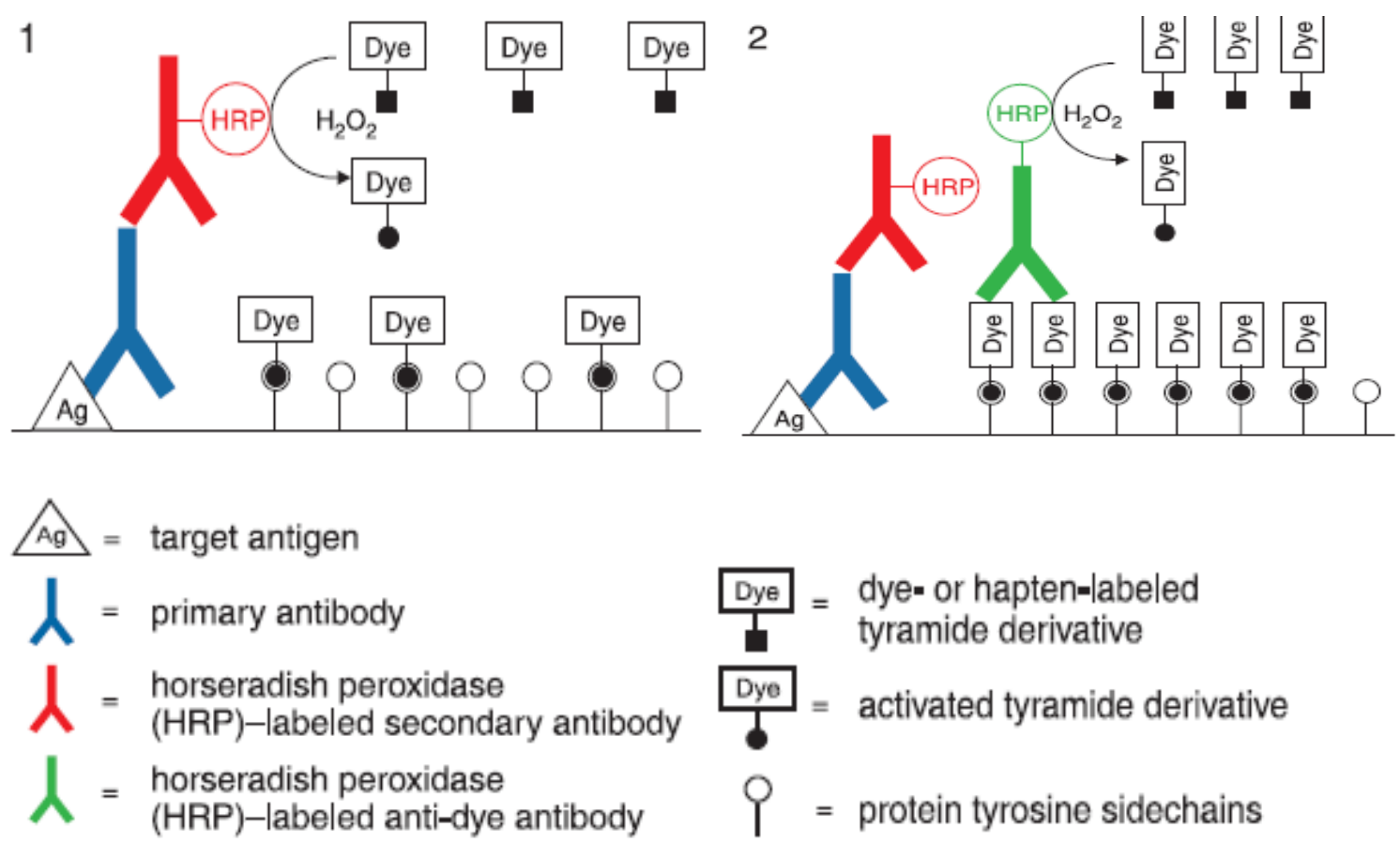

$$
\begin{aligned}
& \text { Dye = } \begin{array}{l}
\text { dye }- \text { or hapten-labeled } \\
\text { tyramide derivative }
\end{array} \\
& \text { Dye = activated tyramide derivative } \\
& \text { = protein tyrosine sidechains }
\end{aligned}
$$

Figure 2.5: Principle of the tyramide signal amplification method. www.mobitec.de/probes/docs/sections/0602.pdf

Of note, the Olig2 and GFAP antibodies were derived from the same species. To avoid crosstalk between these two targets, the two marker proteins were detected using the tyramide signal amplification (Lin et al.) method. Enhanced sensitivity of the TSA allows lower concentrations of the primary antibody. In principle (Fig. 2.5; 1), the target antigen is detected by a primary antibody, followed by a HRP-labeled secondary antibody in conjunction with a dye-labeled tyramide. In the presence of hydrogen peroxide, HRP converts tyramide into a short-lived, extremely reactive free radical intermediate. This free radical intermediate reacts rapidly with HRP and 
covalently binds to electron-rich regions of adjacent proteins (predominantly tyrosine residues) (Fig. 2.5; 2).

After antigen retrieval, fixed tissues were incubated in $3 \% \mathrm{H}_{2} \mathrm{O}_{2}$ in PBS for $1 \mathrm{~h}$ and for $10 \mathrm{~min}$ in Avidin-Biotin solution. After washing with PBS, the slides were blocked in $1 \%$ donkey serum in PBS for $1 \mathrm{~h}$ and incubated overnight with the first primary antibody (Olig2 [rabbit polyclonal]). The next day, tissues were exposed to the secondary antibody (biotylinated-anti-rabbit) for $1 \mathrm{~h}$, incubated with HRP for $1 \mathrm{~h}$ and finally with tyramide-coupled-Alexa 555 for $5 \mathrm{~min}$. To detect mature oligodendrocytes and astrocytes, the slides were again blocked for $20 \mathrm{~min}$ and incubated overnight with the primary antibodies NogoA (mouse) and GFAP (rabbit, polyclonal). The next day, slides were washed with PBS and incubated with the secondary antibodies Alexa 488 (anti-mouse) and Cy5 (anti-rabbit). Cell nuclei were stained with DAPI for $10 \mathrm{~min}$. After washing with PBS, the sections were mounted using fluorescence-mountingmedium.

\subsubsection{Immunofluorescence of cultured cells}

Double immunofluorescence stainings were performed to evaluate the cell densities and morphology of primary astrocyte and oligodendrocyte cultures. I used GFAP and O4 (a sulfatide, expressed in OPCs and mature oligodendrocytes) for astrocyte cultures and $\mathrm{O} 4$ and MBP (expression of MBP indicates differentiated, myelinating oligodendrocytes) for oligodendrocyte cultures.

First, fixed cells were blocked with 10\% FCS in PBS and 0.5\% Tween for 30min and then exposed overnight to the first primary antibody (MBP or GFAP). The next day the cover slips were incubated overnight with the second primary antibody O4. Afterwards, fixed cells were incubated with the secondary antibodies Cy 3 anti-rabbit (binds to MBP and GFAP) and Alexa 488 anti-mouse (binds to O4) for 1.5hrs. Then the cells were washed with PBS and subsequently, nuclei were stained with DAPI for $15 \mathrm{~min}$. After this procedure the cover slips were washed again with $\mathrm{PBS}$ and $\mathrm{H}_{2} \mathrm{O}$ and mounted using fluorescence-mounting medium. 


\subsubsection{TUNEL staining}

Terminal deoxynucleotidyl transferase dUTP nick end labeling (TUNEL) is a method for detecting severe DNA damage or fragmentation resulting from apoptotic signaling cascades or, in some cases also, necrotic cell death. The enzyme terminal deoxynucleotidyl transferase identifies nicks in the DNA and catalyzes the addition of labeled dUTPs.

Deparaffinized sections were rinsed twice with TBS and incubated with Proteinase K for $15 \mathrm{~min}$ at $37^{\circ} \mathrm{C}$. Subsequently, slides were washed 3 times using TBS buffer and once with cold TBS for $5 \mathrm{~min}$. $70 \mu \mathrm{l}$ of IST-working solution was applied to each section. Sections were incubated horizontally in a humid chamber for $1 \mathrm{~h}$ at $37^{\circ} \mathrm{C}$. Afterwards slides were rinsed 5 times with TBS buffer and blocked in 10\% FCS in PBS for $15 \mathrm{~min}$ at room temperature. Then Digoxigenin-AP antibody diluted in $10 \%$ FCS in PBS was applied to the sections and incubated for $1 \mathrm{~h}$ at room temperature. After washing the slides 4 times with TBS, the stains were developed using NBT/BCIP solution, nuclei were stained and sections were mounted using Aquamount.

\subsubsection{In situ hybridization (ISH)}

In situ hybridization is a method to localize a specific DNA or RNA sequence in tissue sections using a labeled complementary DNA or RNA strand (probe).

A PLP RNA probe was generated using linearized PLP plasmid DNA and Digoxigenin-labeled nucleotides.

First sections were deparaffinized and hydrated by performing graded xylene and isopropyl steps and postfixed with 4\% PFA. Then sections were rinsed 3 times with TBS and incubated with $0.2 \mathrm{M} \mathrm{HCl}$ for $10 \mathrm{~min}$ at room temperature to denaturate proteins. After rinsing with TBS, Proteinase $\mathrm{K}$ was applied to the tissue section for $20 \mathrm{~min}$ at $37^{\circ} \mathrm{C}$ to uncover mRNA by digesting proteins. Subsequently slides were rinsed 3 times with TBS and $5 \mathrm{~min}$ with cold TBS, to stop the digestion, and incubated, for acetylation, in $0.5 \%$ acetic anhydride for $10 \mathrm{~min}$ on a shaker. Afterwards the slides were rinsed again 3 times with TBS, dehydrated and exposed to chloroform for $3 \mathrm{~min}$. Then slides were dried off and incubated for $30 \mathrm{~min}$ at $55^{\circ} \mathrm{C}$ without running dry. Afterwards the hybridization-mix was added to the sections and 
incubated for $4 \mathrm{~min}$ at $95^{\circ} \mathrm{C}$ and at $62^{\circ} \mathrm{C}$ overnight. The next day the sections were rinsed twice for $10 \mathrm{~min}$ with washing buffer 1 at room temperature and with washing buffer 2 at $65^{\circ} \mathrm{C}$. After rinsing with TBS again sections were blocked with $10 \%$ FCS in BBR (Boehringer Blocking Reagent) for $15 \mathrm{~min}$ and incubated with an alkaline phosphatase coupled anti-Digoxigenin Ab diluted in blocking mix for 1h. Then the slides were rinsed 3 times with TBS, and the color reaction was developed using NBT/BCIP solution overnight. Afterwards double immunohistochemistry for GFAP and PLP protein was performed and sections were mounted using Aquamount.

\subsection{Statistical analysis}

Graphs were visualized with GraphPad Prism ${ }^{\circledR}$ for Windows. Normality of distribution was verified by the Kolmogorow-Smirnov-test. For statistical calculations comparing two groups, unpaired t-tests were performed. If three or more groups were compared, one-way analysis of variance was carried out. A probability value of less then 0.05 was considered significant. All data are given as mean \pm SEM. 


\section{$3 \quad$ Results}

\section{PART 1}

3.1

Modeling Neuromyelitis optica (NMO) in vitro and in vivo

\subsubsection{Focal NMO model}

\subsubsection{Astrocyte depletion by $\mathrm{rAb}-53$ is complement dependent}

To study the pathogenesis of NMO-like lesions in vivo a focal NMO model in naïve rats was developed. Animals $(n=7)$ were focally injected $(1 \mu l)$ into the motorcortex and/or corpus callosum with the human $\mathrm{rAb}-53(2.5 \mathrm{mg} / \mathrm{ml})$ diluted in human serum, with human serum alone or with the human recombinant anti-measles virus antibody 2B4 (ctrl-rAb) $(2.5 \mathrm{mg} / \mathrm{ml})$ diluted in human serum. After $24 \mathrm{hrs}$ the animals were perfused and processed for immunohistochemistry.
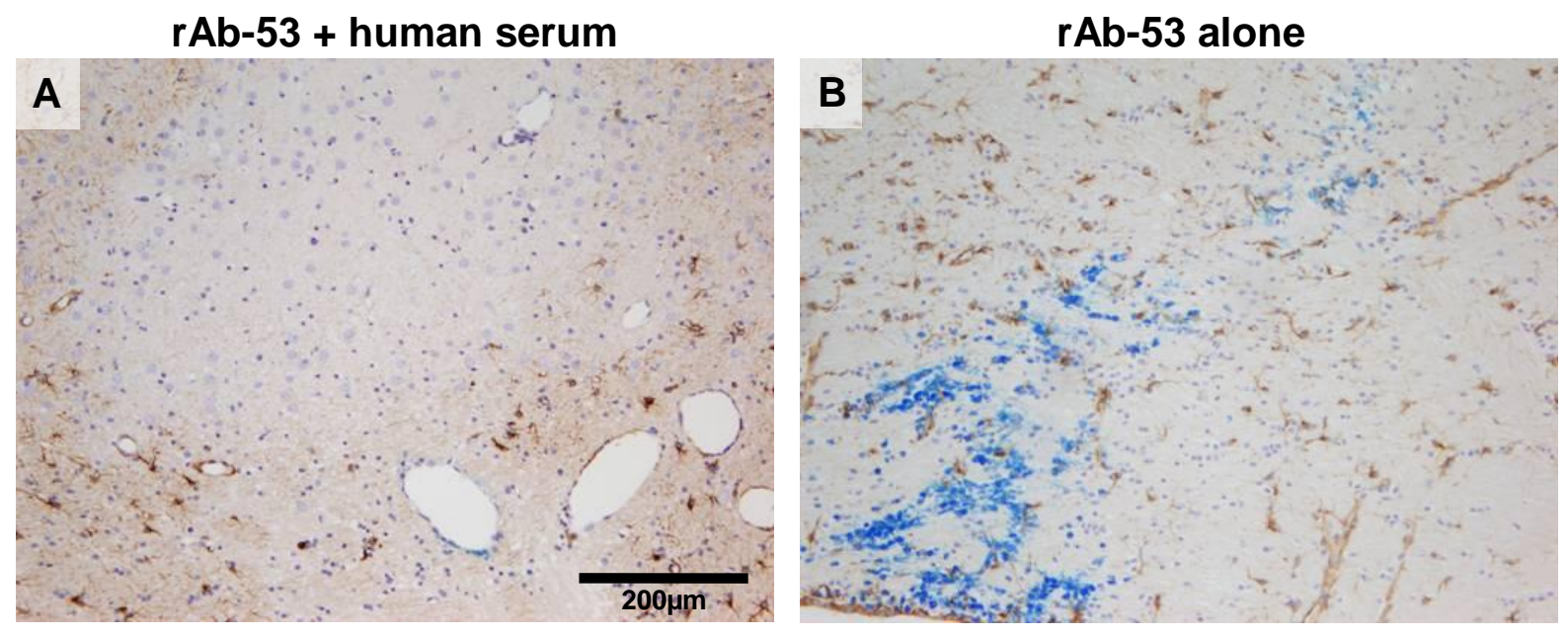

Figure 3.1: Complete astrocyte loss in the cortex of a focally injected rat with $r A b-53$ and human serum (A; GFAP), but not with rAb-53 alone (B; GFAP) $24 \mathrm{hrs}$ after injection. Blue dye marks the injection site.

Massive selective astrocyte loss was observed in GFAP stained sections of the cortex and corpus callosum after injection of $\mathrm{rAb}-53$ and human serum (Fig. 3.1 A). This was also observed using AQP4 immunohistochemistry. Injection of the antibody alone did not generate any astrocyte loss (Fig. $3.1 \mathrm{~B}$ ). The same applied to the 
injections of the ctrl-rAb and human serum, human serum alone or ctrl-rAb alone (not shown).

\subsubsection{Features of astrocyte depleted areas in the focal NMO model}

To compare the lesions of the above described focal NMO model with human histopathology additional immunohistochemical stainings were performed.

24hrs after lesion induction a loss of the astrocytic water channel AQP4, the target of rAb-53, was detected (Fig. 3.2 A). To confirm the selective depletion of astrocytes additional astrocytic markers were used. Both the glutamate transporter EAAT2 (Fig. 3.2. B) and S 100b (data not shown) were depleted to the same extent as AQP4 and GFAP. Additionally NMO-like lesions showed a decrease in the density of mature oligodendrocytes (Fig. 3.7. B) and oligodendrocyte precursor cells (OPCs) (Fig. 3.2. C). Furthermore, astrocyte depleted areas stained positively for human IgG, complement deposits, and APP positive axons (Fig. 3.2 D-F).
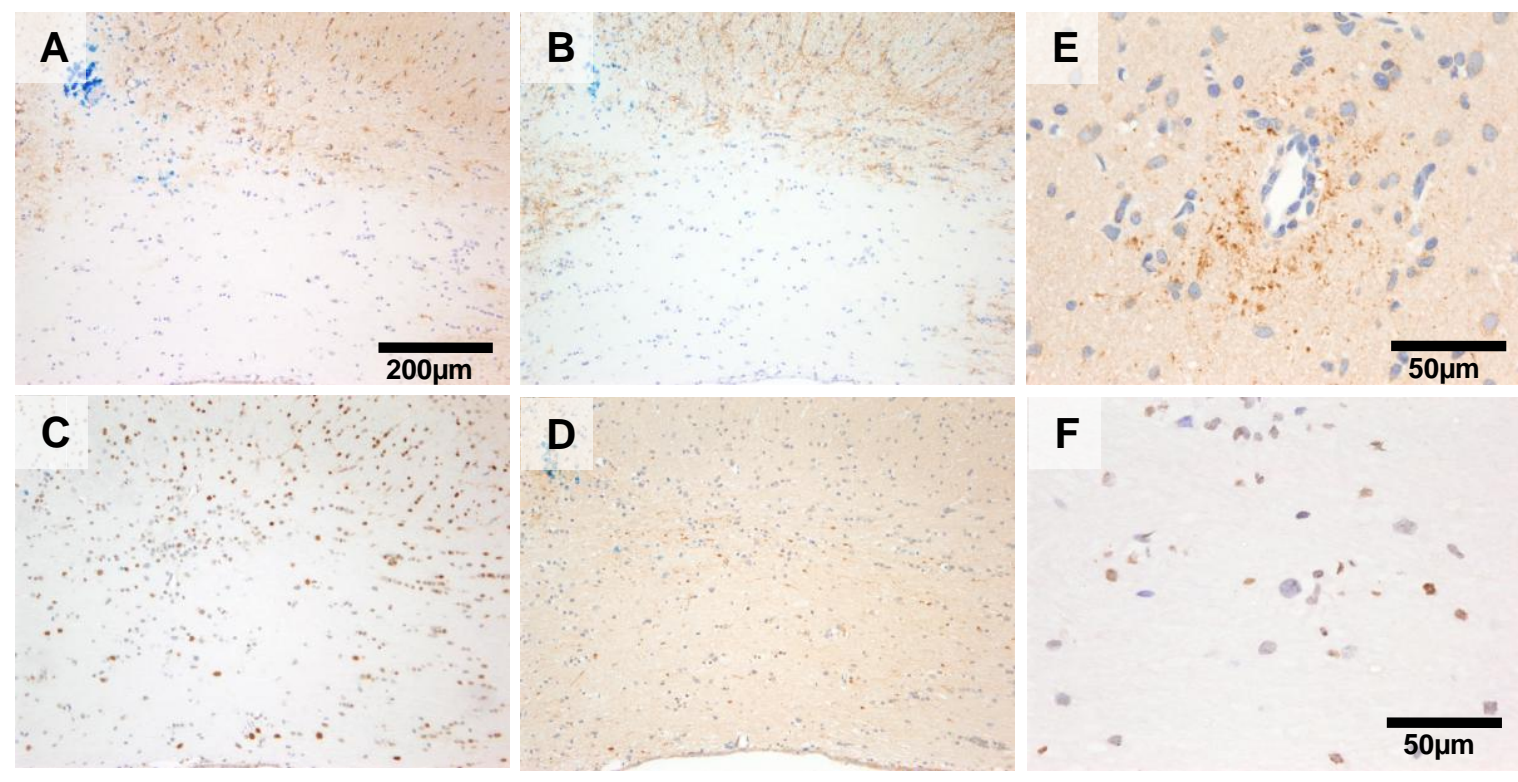

Figure 3.2: Histopathological features of astrocyte depleted areas in the focal NMO model $24 \mathrm{hrs}$ after injection of rAb-53 with human serum. Astrocyte loss is confirmed by AQP4 (A) and EAAT2 (B) IHC, which show the same pattern of depletion. Additionally, NMO-like lesions present with a decrease in OPCs (C; Olig2), IgG depositions (D), axonal pathology (E; APP) and complement depositions ( $F$; C9). Blue dye marks the injection site. 


\subsubsection{Astrocyte death precedes oligodendroglial demise}

To study the time course of NMO-like lesions in the focal NMO model animals were perfused $1 \mathrm{~h}$ and $3 \mathrm{hrs}$ after injection and the tissue processed for immunohistochemistry.

Already $1 \mathrm{~h}$ after antibody injection first signs of astrocyte depletion were observed (Fig. 3.3 A). At this time point no oligodendrocyte loss or DM was seen (Fig. 3.3 B, C). 3hrs after antibody injection the astrocyte depleted area was considerably larger, and a prominent loss of oligodendrocytes but still no DM was revealed (Fig. 3.3 D-F). Oligodendrocyte death was confirmed by a degradation of oligodendrocyte processes and nuclear fragmentation (Fig. 3.3 E, small box).

\section{1h after focal rAb-53 injection}

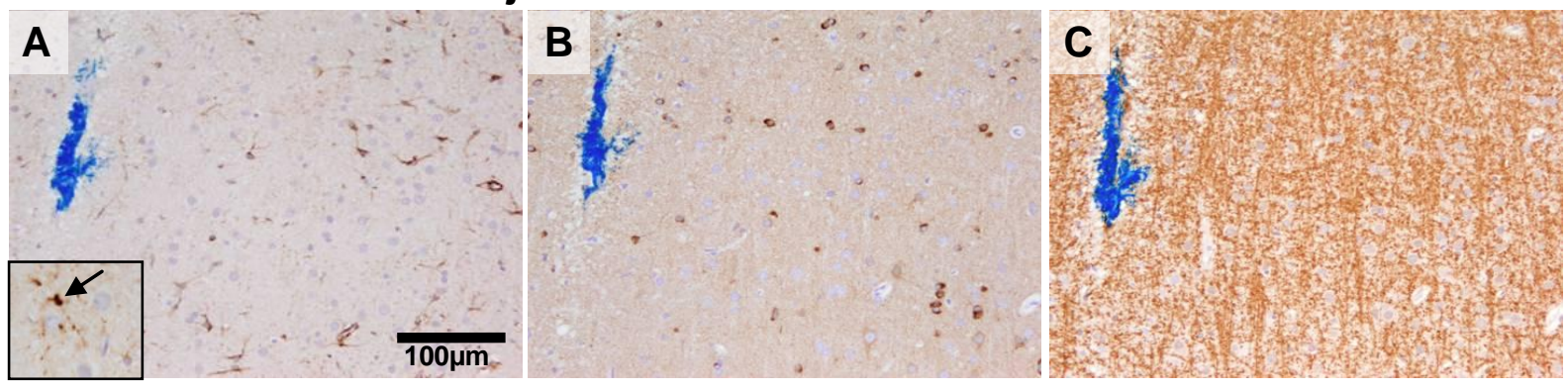

\section{3hrs after focal $\mathrm{rAb}-53$ iniection}

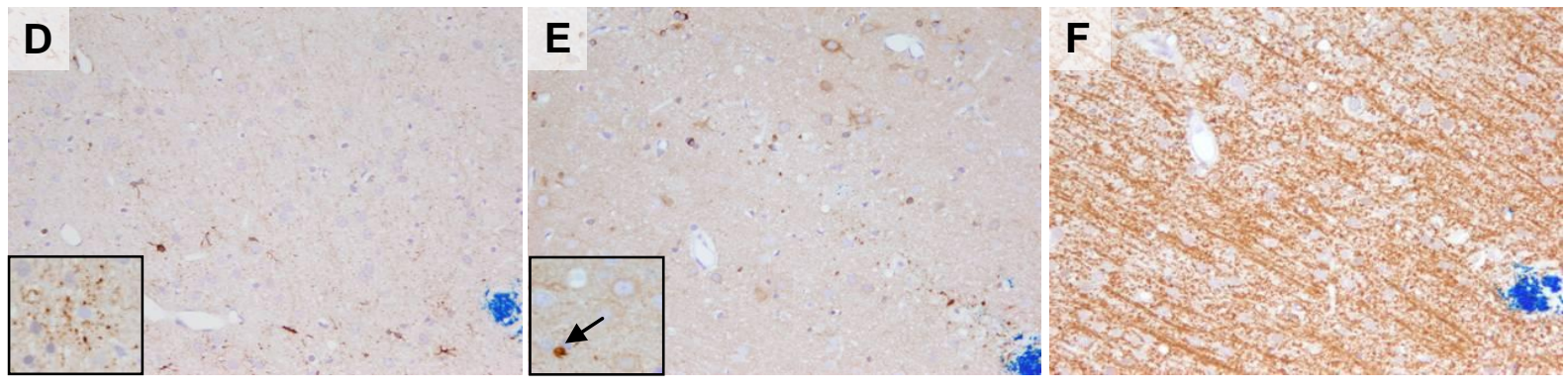

Figure 3.3: Early evolution of focal NMO-like lesions. $1 \mathrm{~h}$ after focal injection astrocyte death is already apparent (A; GFAP), but no loss of oligodendrocytes (B; NogoA) or DM (C; MBP) is observed. 3hrs after injection astrocytes are depleted (D; GFAP) and oligodendrocyte death is already visible (E; NogoA), but no DM is detected (F;MBP). In small boxes of $A$ and $D$ dying astrocytes and in $E$ a dying oligodendrocyte are depicted. Blue dye marks the injection site. 


\subsubsection{Time course of inflammatory cell infiltration}

ED1 immunohistochemistry for activated microglia/macrophages and chloroacetate esterase staining for granulocytes were performed to type inflammatory cells in the focal NMO model. The numbers of activated microglia/macrophages and granulocytes were evaluated at $1 \mathrm{~h}, 3 \mathrm{hrs}, 24 \mathrm{hrs}$ and one week after rAb-53 injection. After $1 \mathrm{~h}$ and 3hrs no infiltration of ED1 positive cells was observed (Fig. 3.4 A+B). After $24 \mathrm{hrs}$ only few activated microglia/macrophages were seen (Fig. 3.4 C). However, numbers of ED1 positive cells rose massively after one week (Fig. 3.4 D). In contrast many granulocytes were seen $24 \mathrm{hrs}$ after antibody injection at the border of focal NMO-like lesions (Fig. 3.5 C). Note that at time points $1 \mathrm{~h}$, 3hrs and one week nearly no granulocytes were detected (Fig. 3.5 A, B, D).

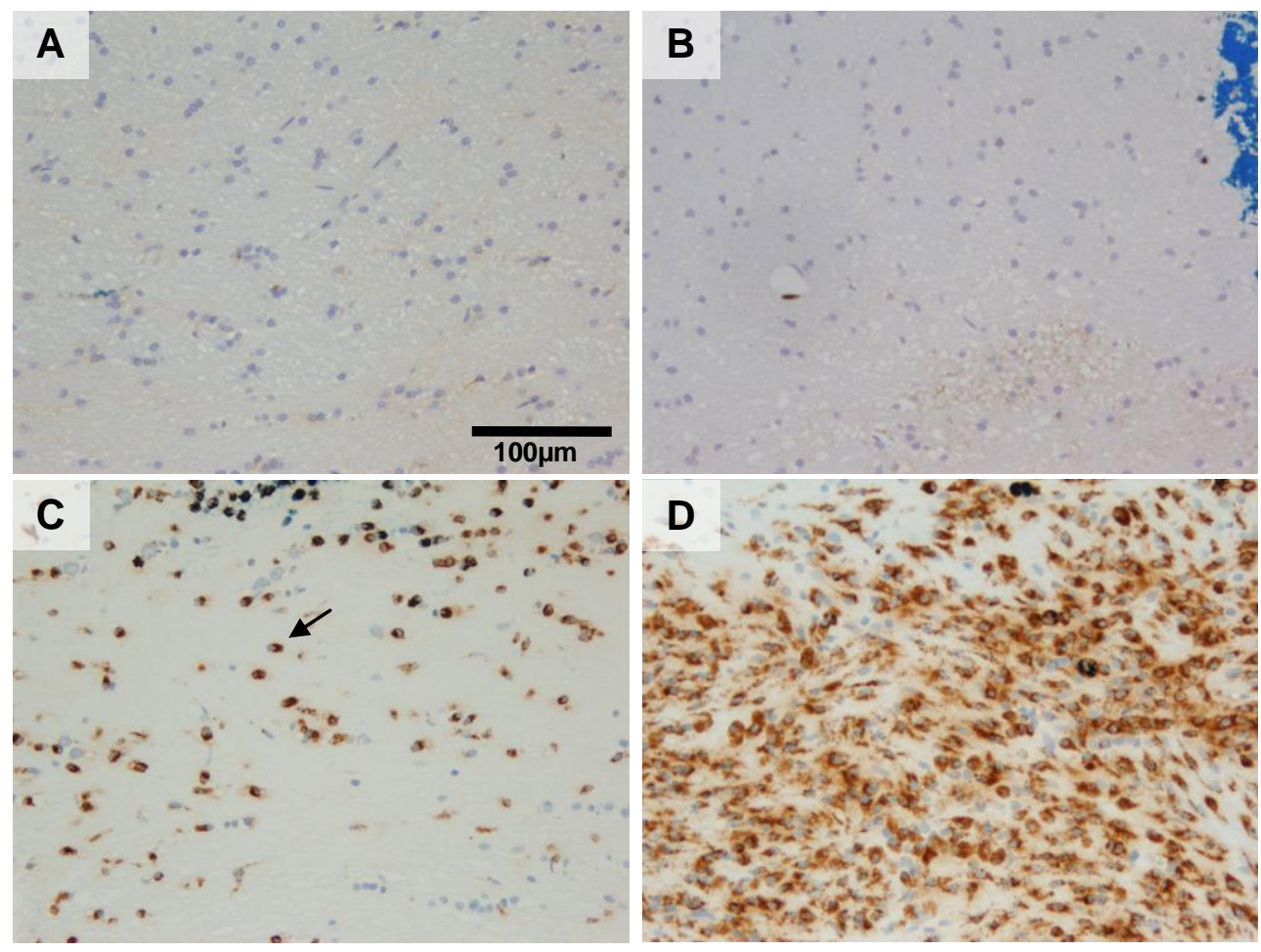

Figure 3.4: Time course of ED1 positive macrophages/activated microglia in focal NMO-like lesions. $1 \mathrm{~h}(\mathrm{~A})$ and $3 \mathrm{hrs}(\mathrm{B})$ post injection nearly no ED1 positive cells were detected. After $24 \mathrm{hrs}$ numbers of infiltrating macrophages/activated microglia had increased $(C)$, but the highest density of ED1 positive cells was only reached after one week (D). Arrow indicates ED1 positive cell. Blue dye marks the injection site. 

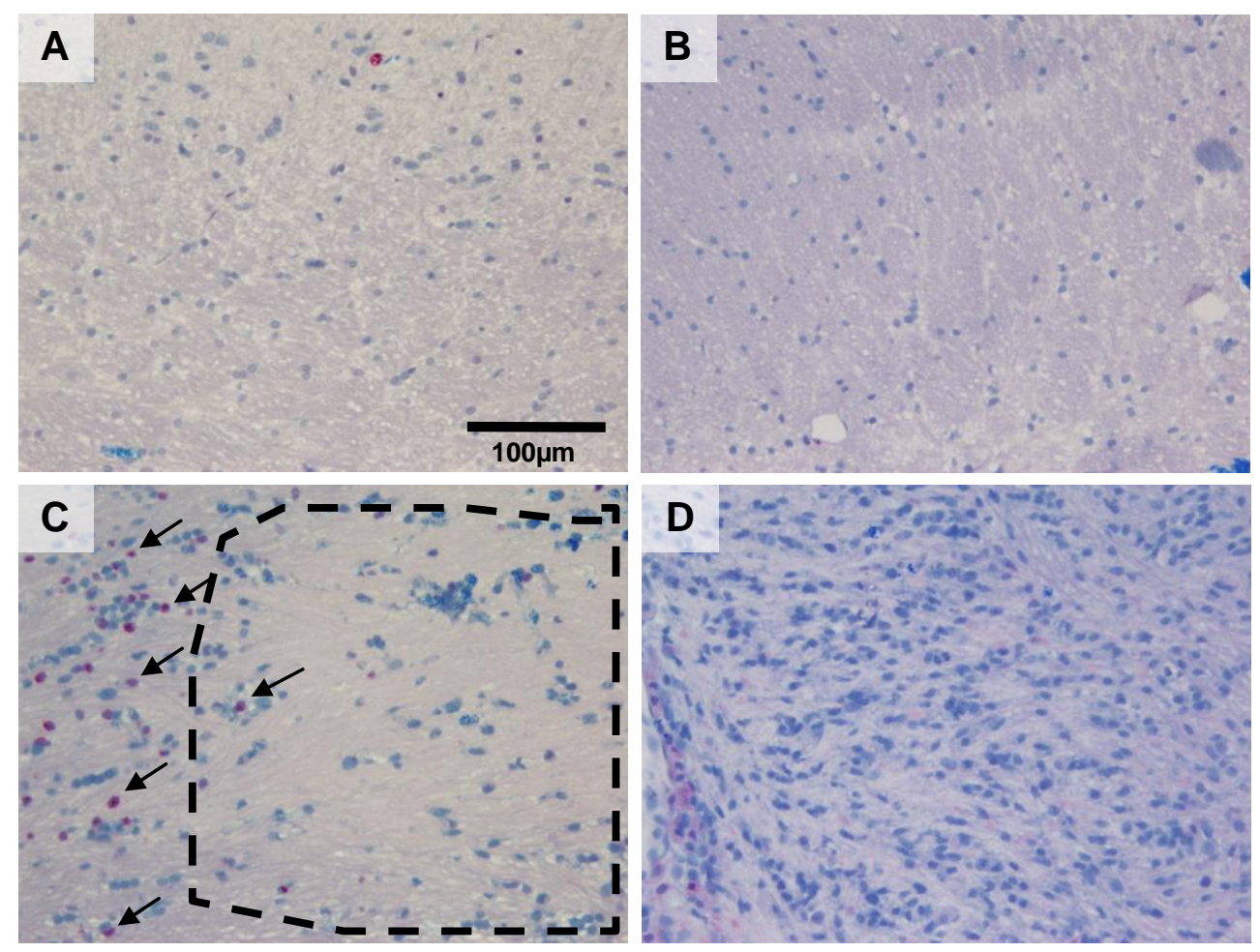

Figure 3.5: Time course of granulocytes in focal NMO-like lesions. 1h (A) and 3hrs (B) post injection nearly no granulocytes (pink) are detected. After 24hrs highest numbers of infiltrating granulocytes are observed (C), which dramatically decrease one week later (D). Dotted line marks astrocyte depleted area. Granulocytes are indicated with arrows. Blue dye marks the injection site.

\subsubsection{Rapid repopulation of astrocyte depleted focal brain lesions}

The evaluation of size, persistence and cellular repopulation of focal NMO-like lesions was performed on GFAP-stained sections at $24 \mathrm{hrs}$ and one week after antibody administration.

The area of astrocyte depletion was significantly larger in animals injected with $\mathrm{rAb}$ 53 + human serum than with the ctrl-rAb + human serum ( $P=0.0004)$ (Fig. 3.6, upper graph, $A+B)$. After one week, astrocytes significantly repopulated the focal NMO-like lesions $(P=0.0041)$ compared to $24 \mathrm{hrs}$. Despite this, the size of the astrocyte free area was still larger in animals injected with $r A b-53$ than with ctrl-rAb after one week $(P=0.0036)$ (Fig. 3.6, lower graph, $C+D$ ). 

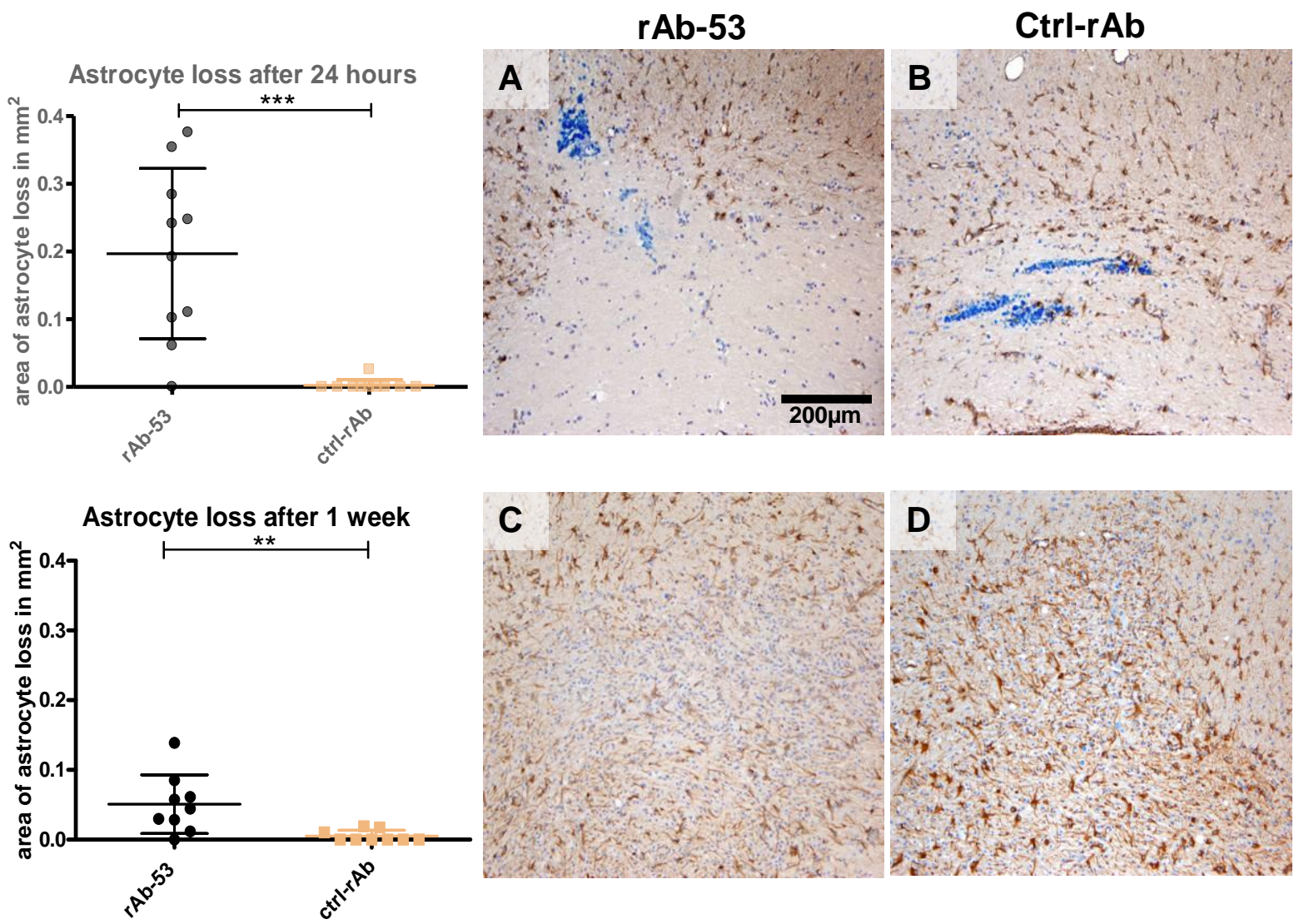

Figure 3.6: Focal NMO-like lesions are in part repopulated by astrocytes already one week after rAb-53 injection. The upper graph shows significant astrocyte loss with rAb-53 and human serum compared to ctrl-rAb with human serum $(P=0.0004)$. The lower graph reveals astrocyte loss after one week $(P=0.0036)$. Astrocytes repopulated the lesion area, but the size of the astrocyte free area is still significantly larger compared to ctrl-rAb injected animals. GFAP IHC confirms the astrocyte loss $24 \mathrm{hrs}$ after injection of $r A b-53$ with human serum (A). Injections with ctrl-rAb and human serum show only moderate astrogliosis after $24 \mathrm{hrs}$, but no astrocyte depletion (B). After one week NMO-like lesion are in part repopulated (C), and ctrl injections show reactive astrocytes (D). Blue dye marks the injection site.

\subsubsection{Little oligodendrocyte repopulation and myelin repair one week after focal astrocyte depletion}

Another question in studying focal NMO-like lesions was if oligodendrocytes repopulated the lesions in a time course similar to astrocytes. Animals were sacrificed $24 \mathrm{hrs}$ and one week post injection. NMO-like lesions were stained for myelin (MBP), mature oligodendrocytes (NogoA) and axonal integrity (Bielschowsky silver impregnation).

After 24hrs a massive loss of oligodendrocytes and pale MBP staining were observed, whereas axons were well preserved (Fig. 3.7 A-C). DM was apparent after one week (Fig. 3.7 D). Furthermore, NMO-like lesions were hardly repopulated by 
oligodendrocytes (Fig. 3.7 E). No decrease in axon density was observed (Fig. 3.7 F).

\section{4hrs after rAb-53 injection}

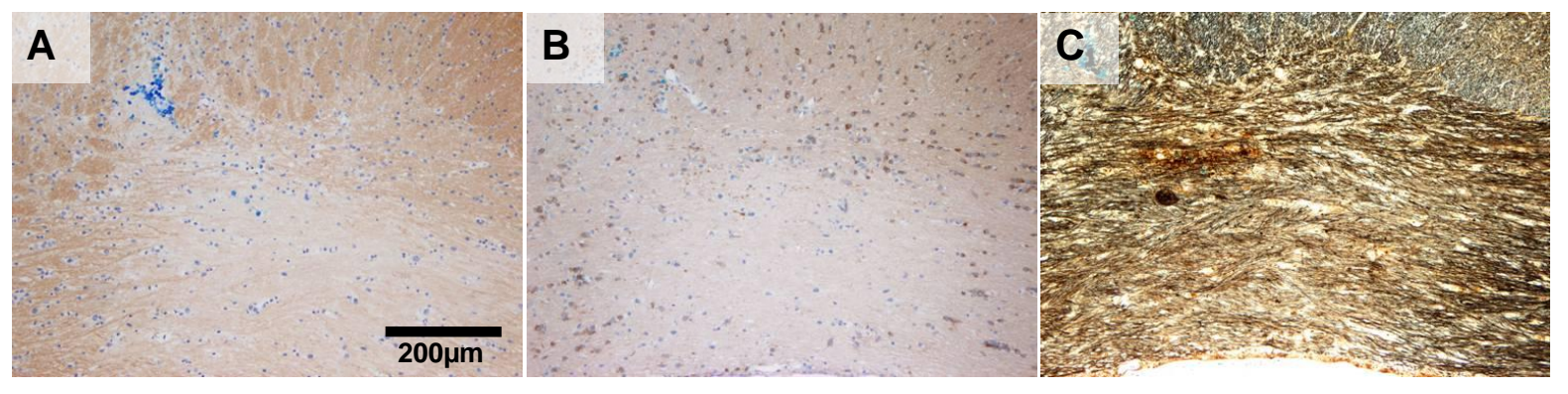

\section{One week after $\mathrm{rAb}-53$ injection}

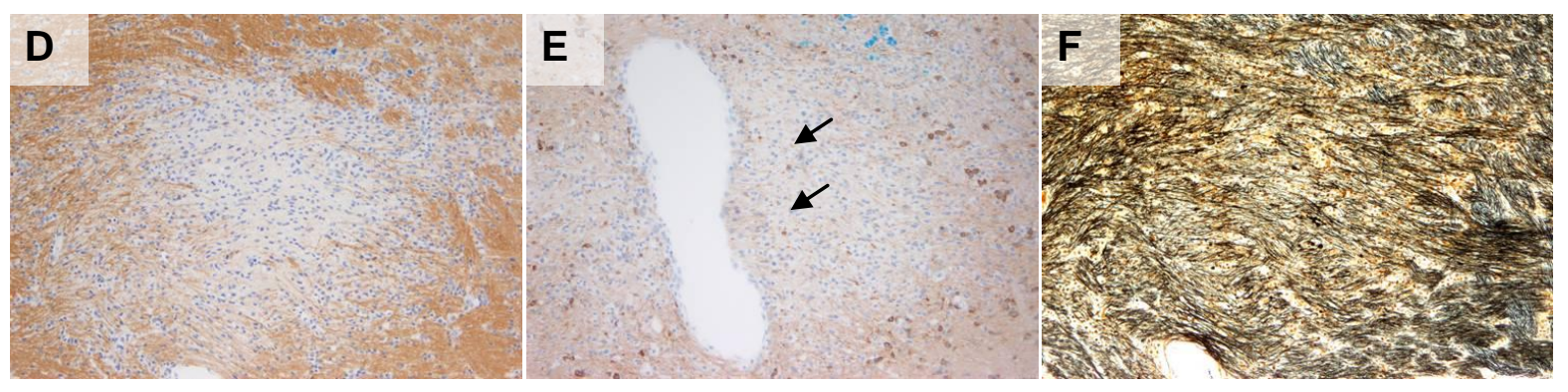

Figure 3.7: Time course of DM and oligodendrocyte repopulation. $24 \mathrm{hrs}$ after $\mathrm{rAb}-53$ and human serum injection a decrease in myelin staining $(A ; M B P)$ and loss of oligodendrocytes $(B)$, but no axonal loss is observed ( $\mathrm{C}$; Bielschowsky silver impregnation). After one week there is still an important decrease in myelin staining (D; MBP), repopulation by oligodendrocytes and RM have not yet occurred (E; NogoA) and no axonal loss is observed (F; Bielschowsky silver impregnation). Arrows: NogoA positive cells. Blue dye marks the injection site.

\subsubsection{Mechanisms of oligodendroglial cell death in vivo}

My results showed that numbers of oligodendrocytes were decreased when astrocytes were depleted in vivo (Fig. 3.8 A). Additionally a dramatic loss in OPC numbers was observed in NMO-like lesions (Fig. $3.8 \mathrm{~B}$ ). But what are the mechanisms of oligodendrocyte and OPC loss, given that AQP4 is not expressed on oligodendroglial cells? On the one hand, factors generated after activation of the complement cascade, released by dying astrocytes or secreted by invading and activated inflammatory cells could cause oligodendroglial cell death. On the other hand, Marignier and coworkers (2010) suggested that glutamate toxicity causes oligodendrocyte death after their incubation with supernatant from astrocytes treated with CSF-IgG from NMO patients. Furthermore, an excess of extracellular ATP has been demonstrated to be toxic for oligodendrocytes (Matute et al., 2007b). 


\section{No treatment}
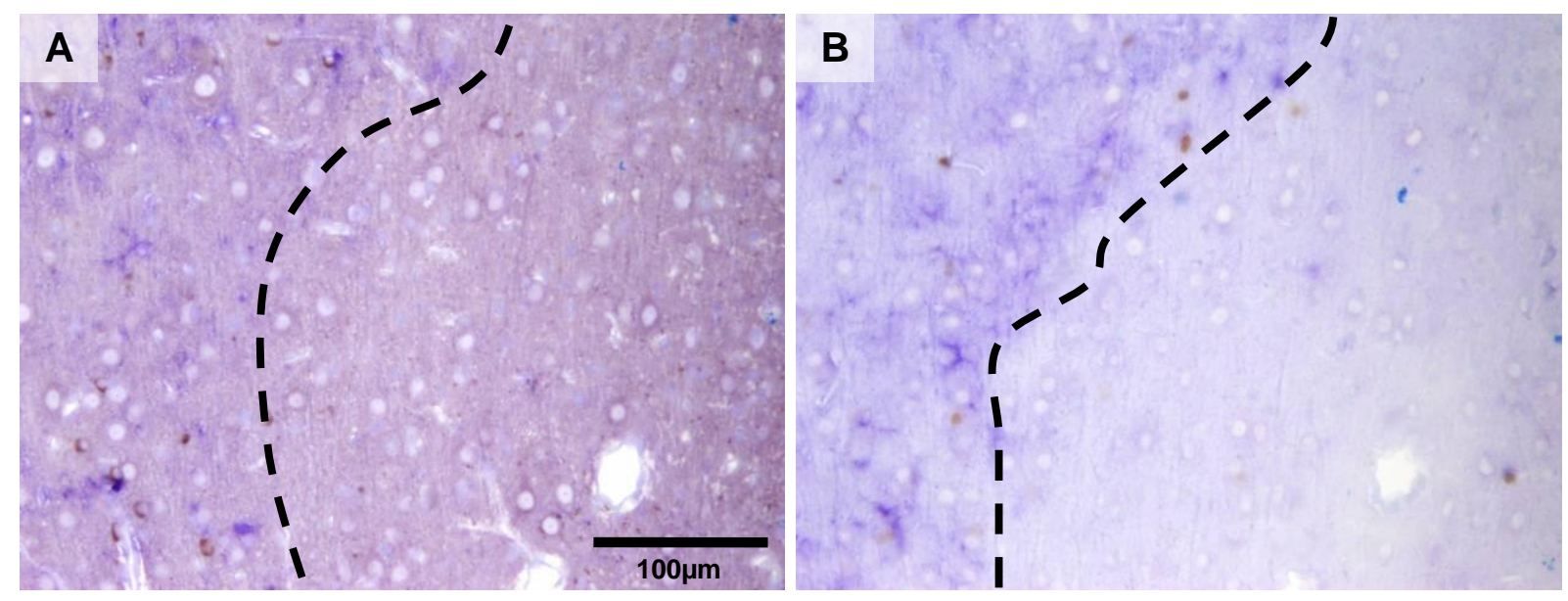

Figure 3.8: Massive loss of oligodendrocytes and OPCs is observed in animals focally injected with rAb-53 and human serum. (A): double staining of NogoA (brown) and GFAP (purple). (B): double staining of Olig2 (brown) and GFAP (purple). Dotted line: astrocyte depleted area.

\subsection{NMDA receptor antagonists}

To test the hypothesis whether oligodendrocyte loss is glutamate and NMDA receptor dependent, animals were focally injected $(2 \mu \mathrm{l})$ with $\mathrm{rAb}-53$, human serum and an NMDA receptor antagonist. Two potent NMDA receptor antagonists, D-AP5 (D-2-Amino-5-phosphonopentanoic acid) and MK-801 ((+) MK-801 hydrogen maleate), in a concentration of $500 \mu \mathrm{mol} / \mathrm{L}$, were used.

Intracerebral injections $(2 \mu \mathrm{l})$ of the receptor antagonists alone did not produce any damage to brain cells. Local treatment with neither D-AP5 nor MK-801 showed any effect on the loss of mature NogoA-positive oligodendrocytes (Fig. 3.9 A+B). Similarly, in our preliminary experiments, no clear-cut effect of the NMDA-receptor antagonists D-AP5 and MK801 on the density of Olig2-positive cells were observed (Fig. $3.9 \mathrm{C}+\mathrm{D}$ ). Further experiments including detailed morphometric evaluations will be performed to substantiate these findings. 


\section{Treatment with D-AP5}
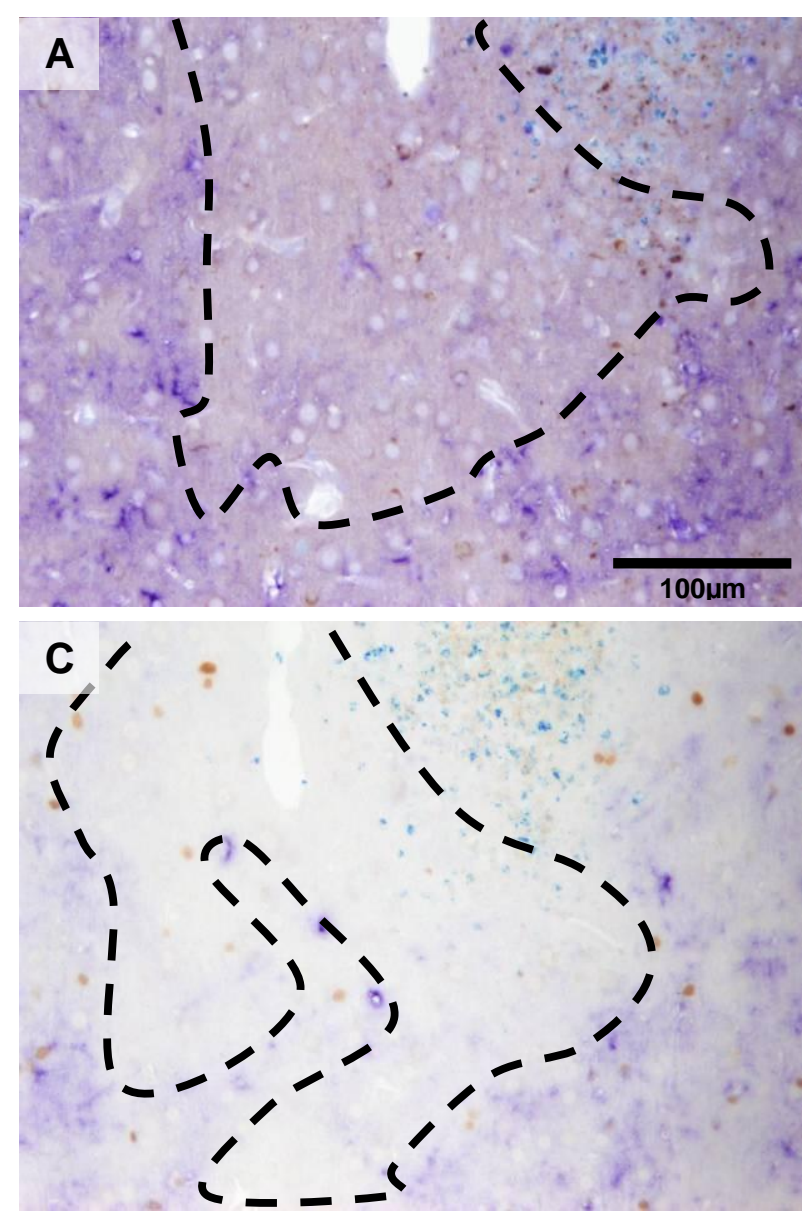

\section{Treatment with MK-801}

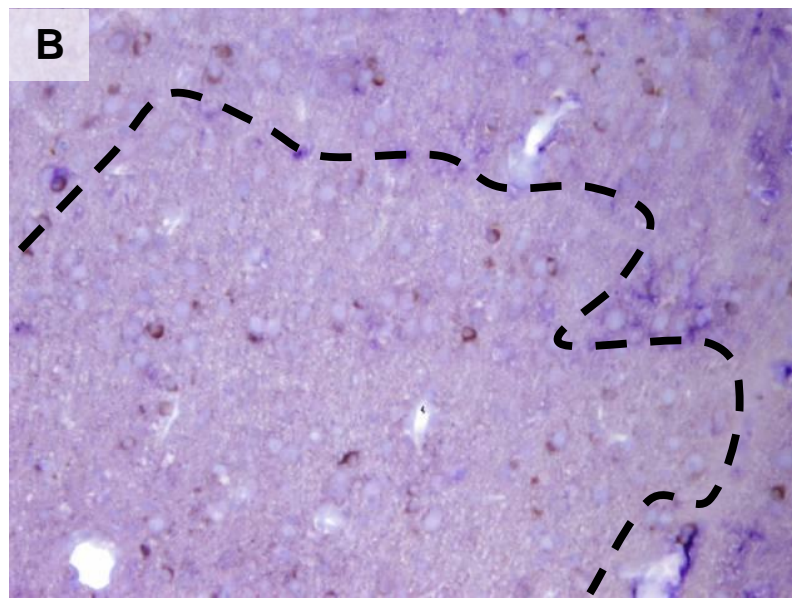

D

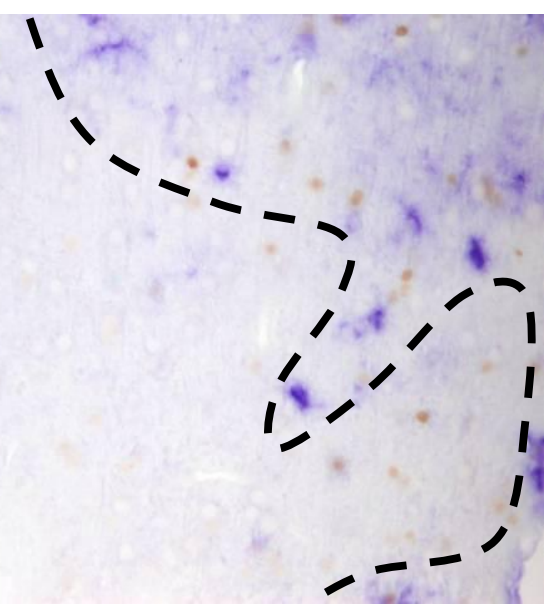

Figure 3.9: NMDA receptor antagonist treatment in focal NMO-like lesions. $(A+C)$ : Injection of rAb-53 with human serum and D-AP5. (B+D): Injection of rAb-53 with human serum and MK-801. $(A+B)$ : double staining of NogoA (brown) and GFAP (purple). (C+D): double staining of Olig2 (brown) and GFAP (purple). Dotted line: astrocyte depleted area.

\subsubsection{2 $\quad \mathrm{P}_{2} \mathrm{X}_{7}$ receptor antagonists}

My in vivo experiments $(\mathrm{n}=2)$ did not confirm a central role of NMDA receptor activation for oligodendrocyte and OPC demise. When astrocytes are lysed, intracellular components are released, among them ATP. In other lesion paradigms, ATP was shown to kill oligodendrocytes due to over activation of their $P_{2} X_{7}$ receptors (Matute et al., 2007b).

To test this hypothesis for focal NMO-like lesions, two $\mathrm{P}_{2} \mathrm{X}_{7}$ antagonists, OATP and BBG, in a concentration of $500 \mu \mathrm{mol} / \mathrm{L}$, were used. Intracerebral injections $(2 \mu \mathrm{l})$ of the $\mathrm{P}_{2} \mathrm{X}_{7}$ antagonists alone did not show any cellular toxicity.

The preliminary qualitative evaluation of focal NMO-like lesions injected with oATP or BBG did not detect overtly increased numbers of mature oligodendrocytes (Fig 3.10 
$A+B$ ) or OPCs (Fig. 3.10 C+D). To further clarify the role of $P_{2} X_{7}$ receptor antagonists on oligodendroglial cell death in NMO-like lesions additional morphometric evaluations are needed.

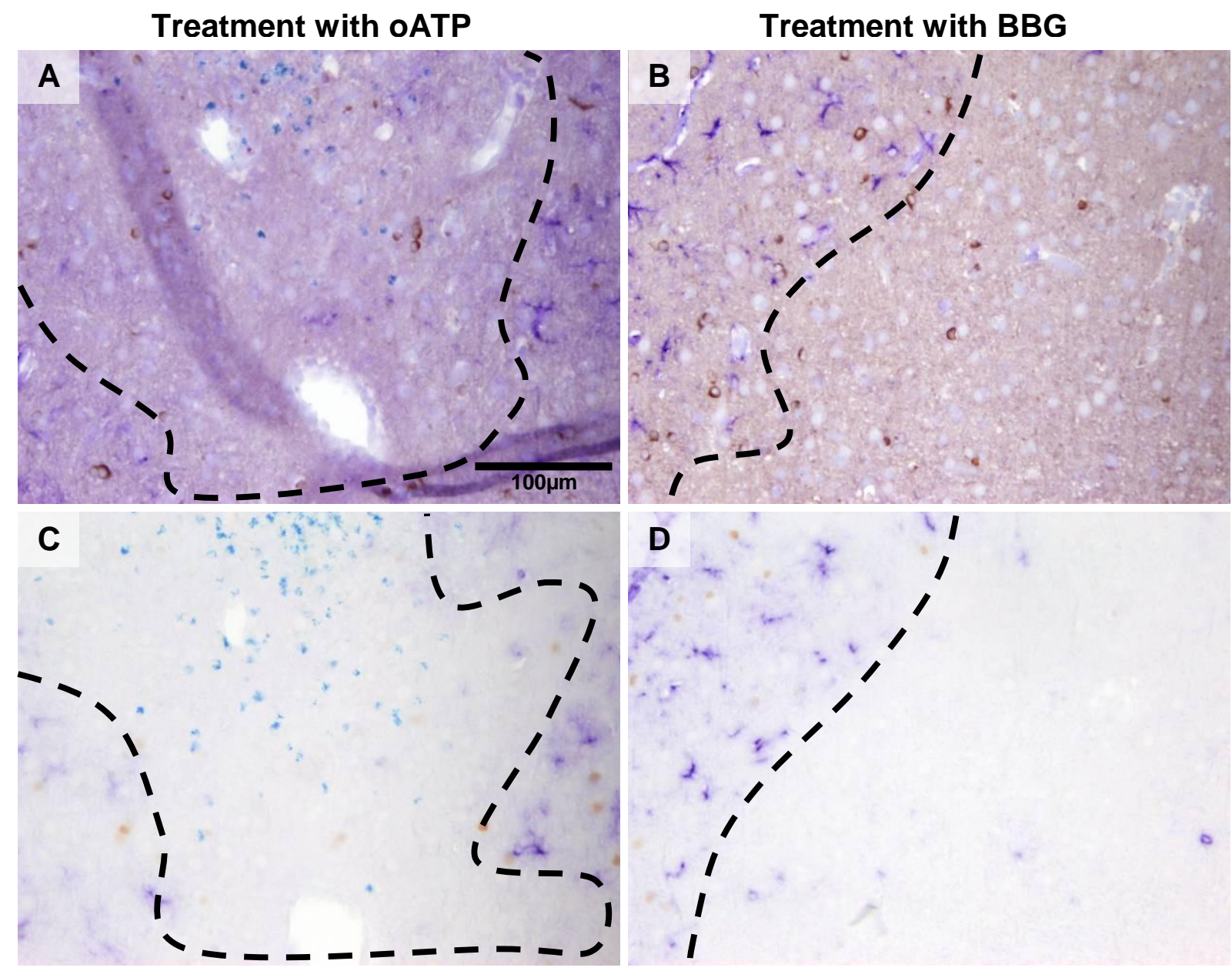

Figure 3.10: $P_{2} X_{7}$ receptor antagonist treatment in focal NMO-like lesions. $(A+C)$ : Injection of $r A b-$ 53 with human serum and $O A T P$. (B+D): Injection of $r A b-53$ with human serum and $B B G$. $(A+B)$ : double staining of NogoA (brown) and GFAP (purple). (C+D): double staining of Olig2 (brown) and GFAP (purple). Dotted line: astrocyte depleted area.

\subsubsection{Antibody-transfer model of NMO (EAE/NMO model)}

\subsubsection{Features of astrocyte depleted areas in rat EAE}

To study the pathogenesis of $\mathrm{rAb}-53$ generated lesions under inflammatory conditions in the spinal cord, the gpMBP $72-85$-EAE model in rats $(n=36)$ was used. At approximatly seven days post immunization animals showed first clinical signs of

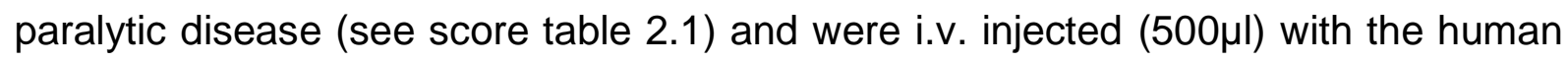
rAb-53 $(5 \mathrm{mg} / \mathrm{ml})$. Control animals were injected $(500 \mu \mathrm{l})$ with PBS or the ctrl-rAb 
$(5 \mathrm{mg} / \mathrm{ml}) .30 \mathrm{hrs}$ after injection animals were sacrificed and tissue was processed for immunohistochemistry. Induction of perivenous brain inflammation is necessary to open the BBB and allow the i.v. injected antibodies to enter the CNS (Linington et al., 1988).

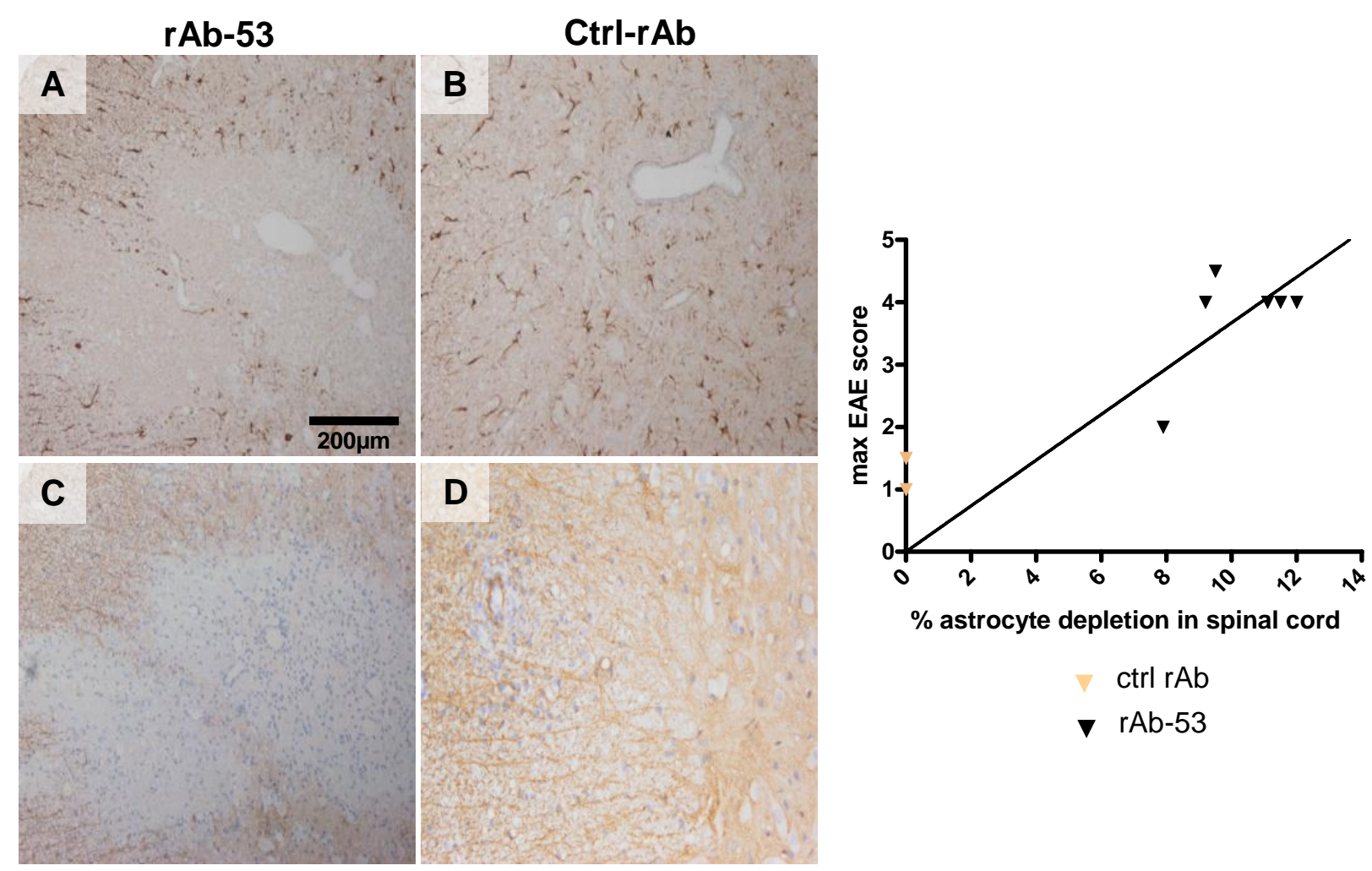

Figure 3.11: Astrocyte depletion in the rat EAE model when i.v. injected with rAb-53 $(A+C)$, but not with ctrl-rAb $(B+D)$. The graph shows a significant correlation between the maximal EAE score and the percentage of astrocyte depleted spinal cord area $(P<0.0001)$. Findings in GFAP staining $(A+B)$ are confirmed by $A Q P 4$ staining $(C+D)$.

Large astrocyte depleted perivenous areas were observed in the spinal cord of $\mathrm{rAb}$ 53 injected animals applying GFAP and AQP4 staining (Fig. 3.11 $A+C$ ). The white and grey matter were affected to a similar extent. In contrast, in control rats, injected with ctrl-rAb, no astrocyte depletion or other cell loss was observed. To confirm that astrocytes are lost (and not only AQP4 is downregulated), stainings against other astrocyte markers, such as S100b and EAAT2 were performed. GFAP and S100b were lost to the same extent as AQP4 and EAAT2, thus confirming astrocyte depletion (data not shown). Animals transferred with rAb-53 developed more severe disease than those injected with ctrl-rAb. The area of astrocyte loss in the spinal cord correlated significantly with the EAE scores maximally obtained (Fig. 3.11). 

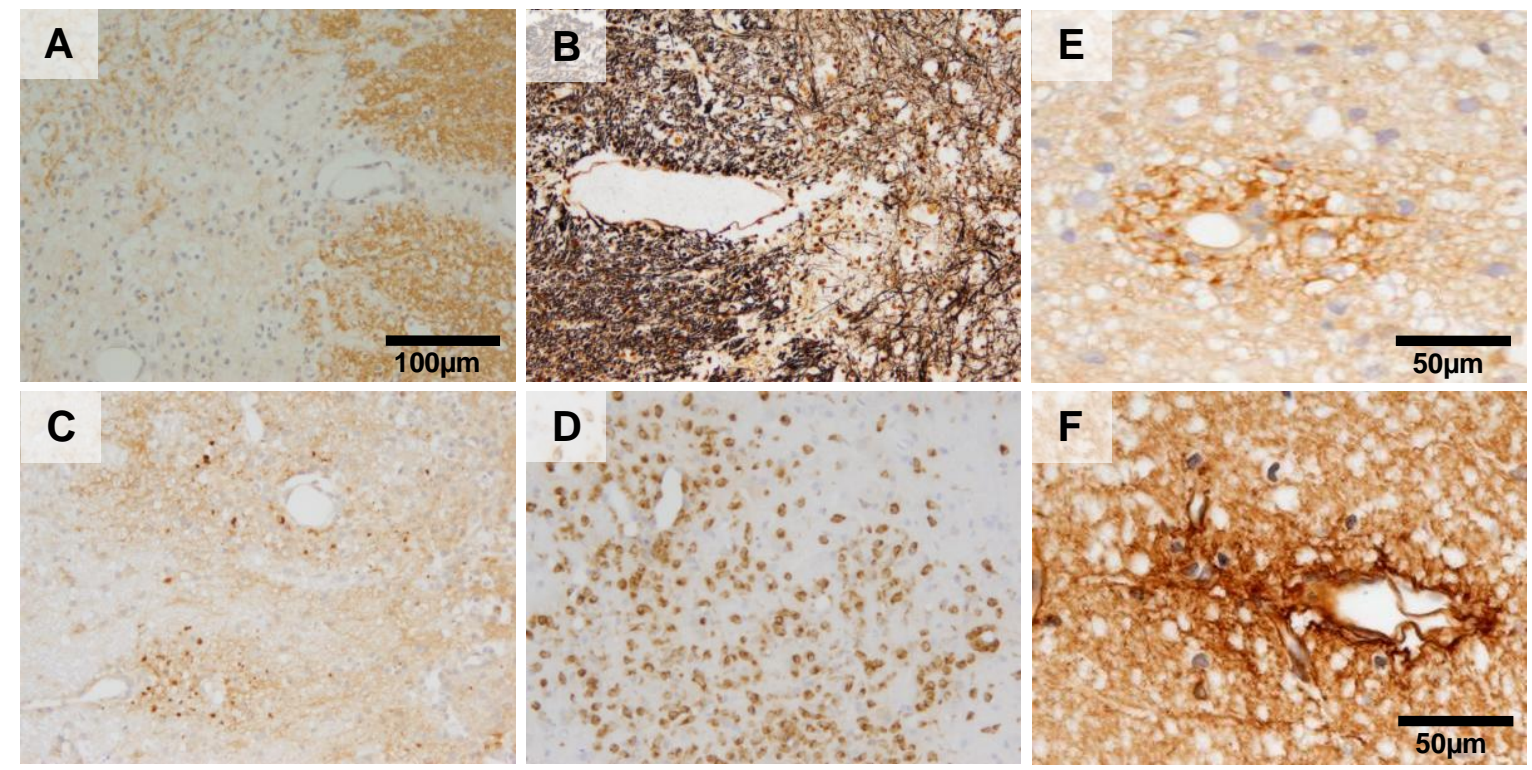

Figure 3.12: Histopathological features of astrocyte depleted areas in the NMO/EAE model $30 \mathrm{hrs}$ after $\mathrm{rAb}-\mathbf{5 3}$ administration. NMO-like lesion show myelin loss (A; MBP), but no axonal loss (B: Bielschowsky silver impregnation). However, disturbances of axonal transport are observed (C; APP). Astrocyte depleted areas also contain macrophages/activated microglia (D; ED1), perivascular complement ( $E$; C9) and IgG depositions ( $F$; human IgG).

30hrs after i.v. antibody transfer, in the EAE/NMO model myelin staining intensity was markedly reduced, but no clear DM was observed. Bielschowsky silver impregnation showed intact axons (Fig. 3.12 A+B). However, APP positive axons were detected in NMO-like lesions, indicating disturbances of axonal transport (Fig. 3.12 C). ED1 staining showed an infiltration by activated microglia/macrophages (Fig. 3.12 D). Perivascular complement components and human IgG depositions were found (Fig. 3.12 E+F).

\subsubsection{Reduction of mature oligodendrocytes in astrocyte depleted areas}

To evaluate whether astrocyte depletion also causes a loss of mature oligodendrocytes in the systemic EAE/NMO model the density of NogoA positive cells in the lesions was determined.

Oligodendrocytes in the white and grey matter were significantly decreased in NMOlike lesions ( $155.5 \pm 10.14 \mathrm{cells} / \mathrm{mm}^{2}$ in WM; $96.5 \pm 4.1 \mathrm{cells} / \mathrm{mm}^{2}$ in GM) compared to animals injected with the ctrl-rAb $\left(222.8 \pm 18.29 \mathrm{cells} / \mathrm{mm}^{2}\right.$ in WM; $224.1 \pm 6$ cells $/ \mathrm{mm}^{2}$ in GM) (Fig. 3.13). However, the loss of oligodendrocytes in grey matter lesions $(P<0.0001)$ was more dramatic than in white matter lesions $(P=0.0166)$. 


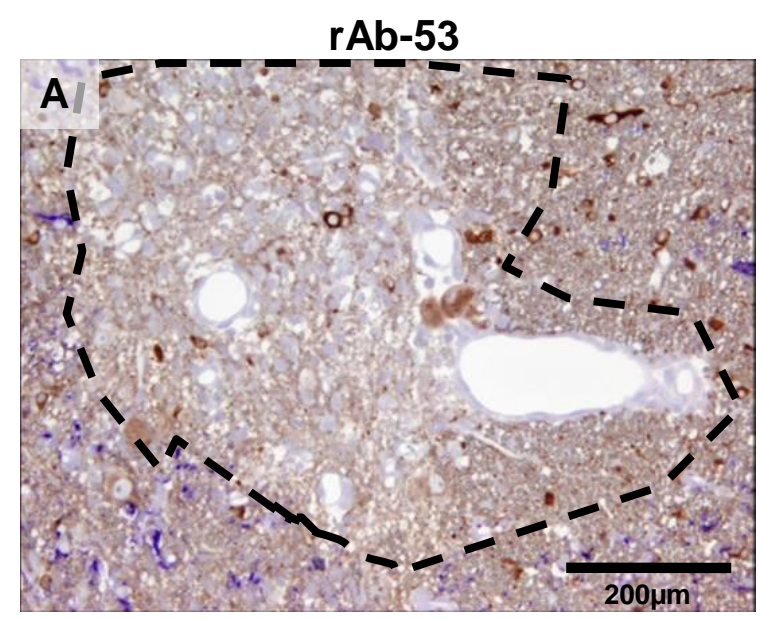

White matter lesions

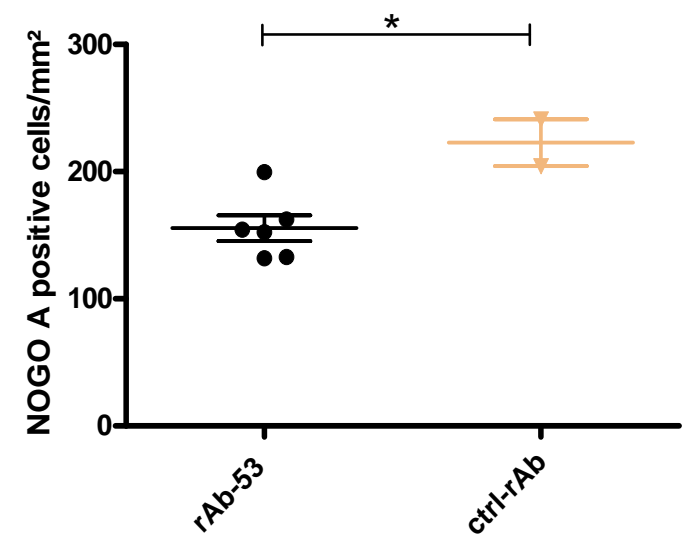

\section{Ctrl-rAb}

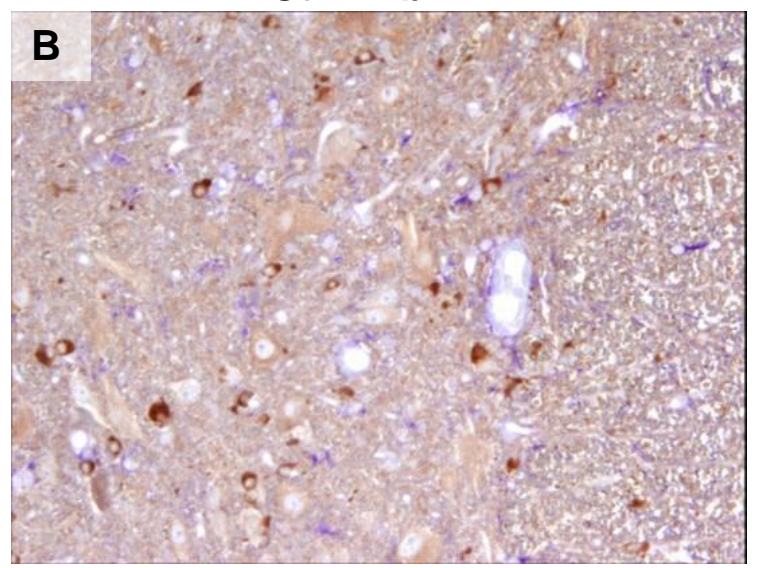

Grey matter lesions

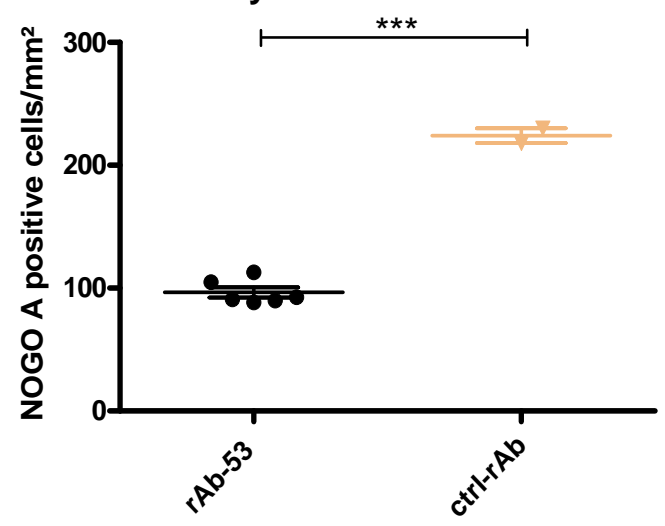

Figure 3.13: NMO-like lesions reveal a decrease in oligodendrocytes 30hrs after antibody administration. Animals injected with $\mathrm{rAb}-53$ show loss of oligodendrocytes in astrocyte depleted areas (A) compared to animals treated with ctrl-rAb (B) in NogoA (brown)/GFAP (purple) doublestainings. Graphs show a significant decrease of NogoA positive cells in white $(P=0.0166)$ and grey matter lesions $(P<0.0001)$. Dotted line: astrocyte depleted area.

\subsubsection{Loss of PLP mRNA positive oligodendrocytes in astrocyte depleted areas}

PLP in situ hybridization on spinal cord sections was performed to underpin the loss of oligodendrocytes in NMO-like lesions. Cells positive for PLP MRNA were determined. Counts were performed on sections costained for PLP protein and GFAP to detect the area of astrocyte loss.

In both, white and grey matter a significant decrease in PLP mRNA positive cells was found in astrocyte depleted areas $\left(106.4 \pm 18.18\right.$ cells $/ \mathrm{mm}^{2}$ in WM; $49.5 \pm 11.75$ cells $/ \mathrm{mm}^{2}$ in GM) compared to spinal cord tissue of control animals $(275.9 \pm 10.27$ cells $/ \mathrm{mm}^{2}$ in WM; $261.8 \pm 32.64$ cells $/ \mathrm{mm}^{2}$ in GM) (Fig. 3.14). Of note, though, the 
reduction of PLP mRNA positive cells $(P=0.0023)$ was higher (Fig 3.14) than the loss of NogoA positive cells (P=0.0166) in white matter (Fig.3.13).

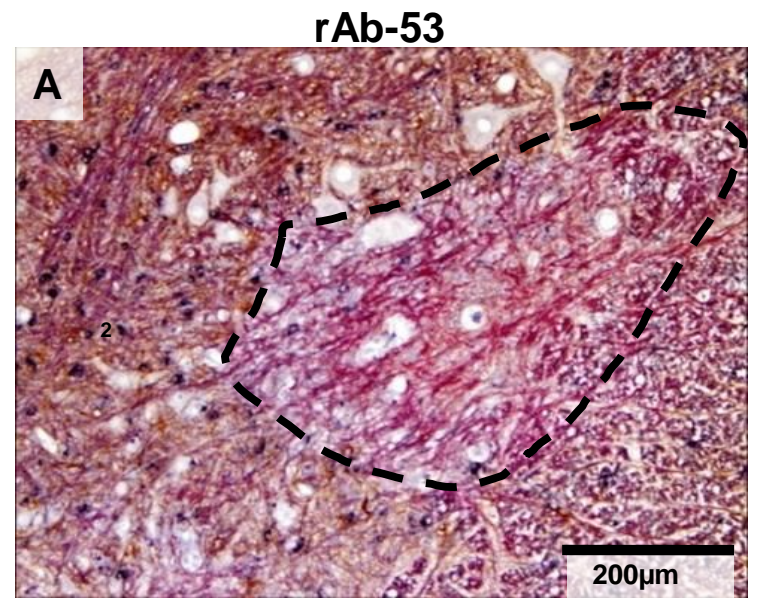

White matter lesions

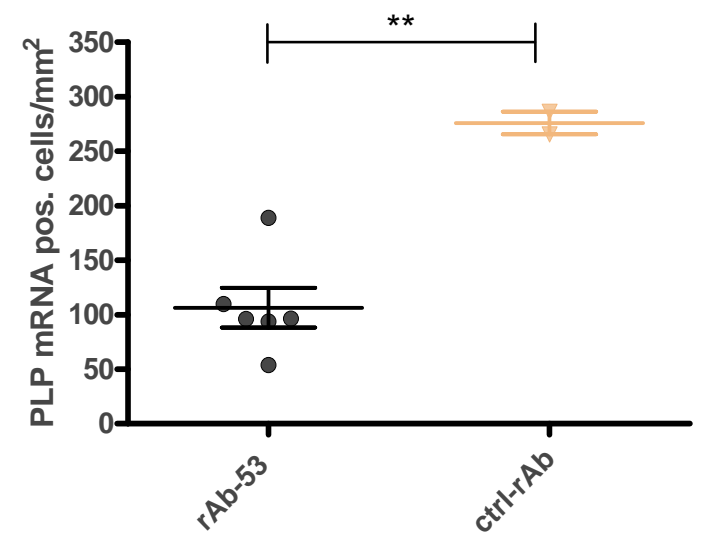

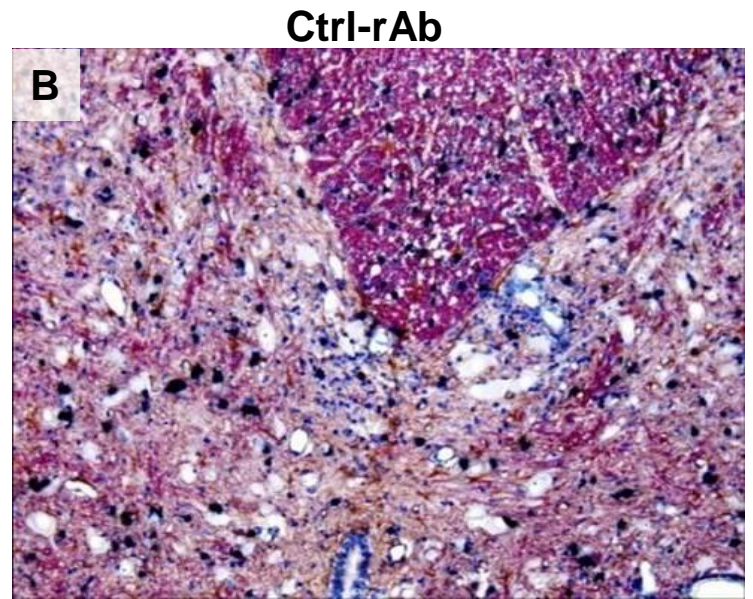

Grey matter lesions

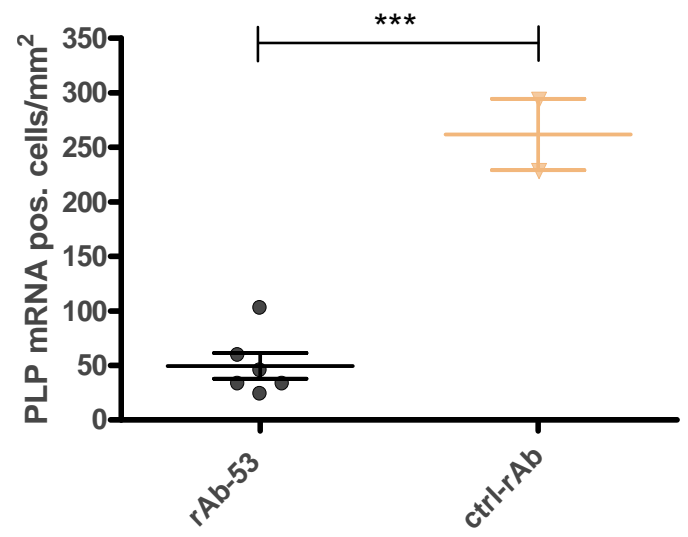

Figure 3.14: Reduction of PLP mRNA positive cells in NMO-like lesions. In situ hybridization of PLP (pink) and GFAP (brown)/PLP (pink) double immunohistochemistry of animals injected with rAb$53(A)$ or ctrl-rAb (B) 30hrs post injection. Graphs show significantly lower numbers of oligodendroglia positive for PLP mRNA in white $(P=0.0023)$ and grey matter lesions $(P=0.0002)$ of animals injected with $\mathrm{rAb}-53$ compared to ctrl-rAb. Dotted line: astrocyte depleted area.

\subsubsection{OPCs are as vulnerable as mature oligodendrocytes}

In the focal NMO model a prominent loss of OPCs in astrocyte depleted areas was observed. To assess whether this was also true for the systemic EAE/NMO model Olig2 staining was performed.

The numbers of Olig2 positive cells were decreased $\left(108.8 \pm 11.7\right.$ cells $/ \mathrm{mm}^{2}$ in WM; $59.4 \pm 7.6 \mathrm{cells} / \mathrm{mm}^{2}$ in $\mathrm{GM}$ ) in astrocyte depleted areas compared to tissue from animals treated with ctrl-rAb $\left(173.9 \pm 27.03\right.$ cells $/ \mathrm{mm}^{2}$ in WM; $154.8 \pm 33.2 \mathrm{cells} / \mathrm{mm}^{2}$ in GM) (Fig. 3.15). These findings were significant in white $(P=0.0398)$ and grey matter lesions $(P=0.0038)$. 
rAb-53

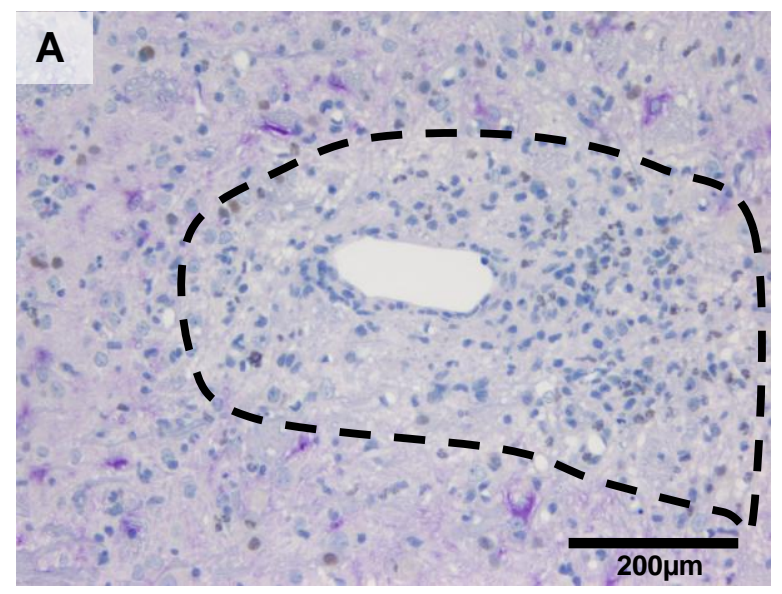

White matter lesions

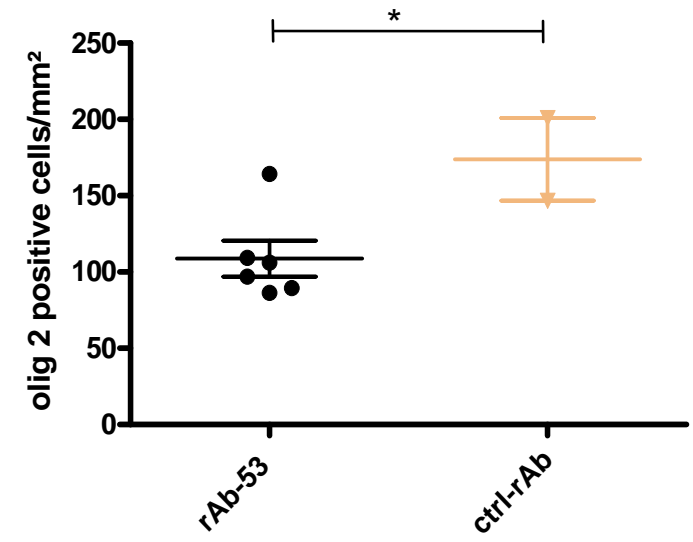

Ctrl-rAb

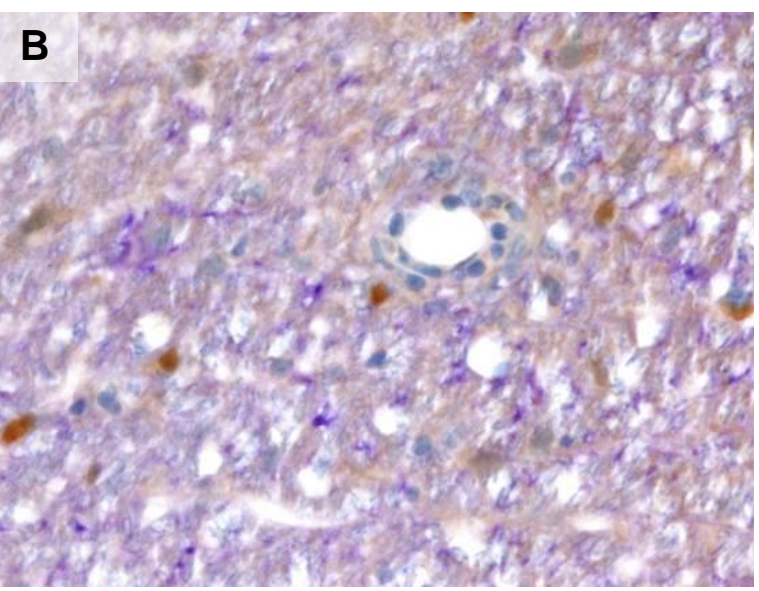

\section{Grey matter lesions}

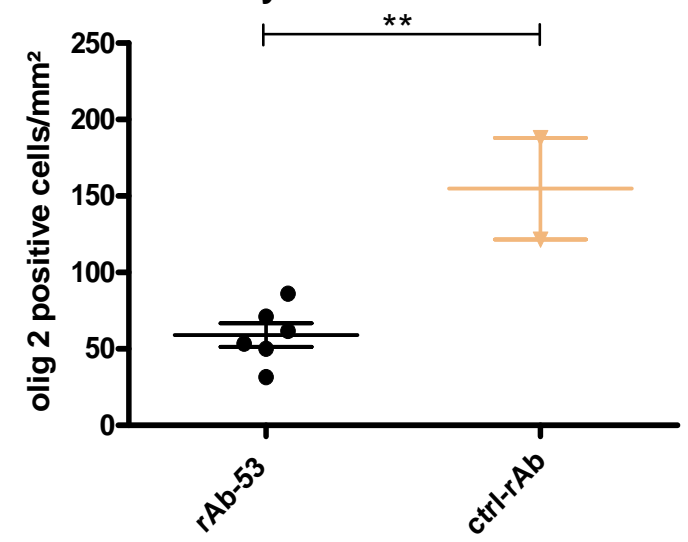

Figure 3.15: Olig2 positive cells are decreased in NMO-like lesions 30hrs after rAb-53 injection.

Olig2 (brown)/GFAP (purple) double IHC of animals injected with rAb-53 (A) or ctrl-rAb (B). Graphs demonstrate a significant loss of Olig2 positive cells in white $(P=0.0398)$ and grey matter lesions $(P=0.0038)$ of animals i.v. injected with $\mathrm{rAb}-53$ compared to ctrl-rAb. Dotted line: astrocyte depleted area.

As in 2.4.3.4 Olig2 antibody also faintly labels nuclei of mature oligodendrocytes. To clearly discern OPCs from mature oligodendrocytes triple IHC for NogoA, Olig2 and GFAP was performed. Cells double positive for Olig2 and NogoA were found in the white and grey matter (Fig. 3.16, small box). To identify immature oligodendrocytes cells that were only positive for Olig2 were counted.

Counting only Olig2 positive cells, a significant decrease in OPCs in NMO-like lesions in the white $(P=0.0114)$ and grey matter $(P=0.0246)$ was observed (Fig. 3.16). 


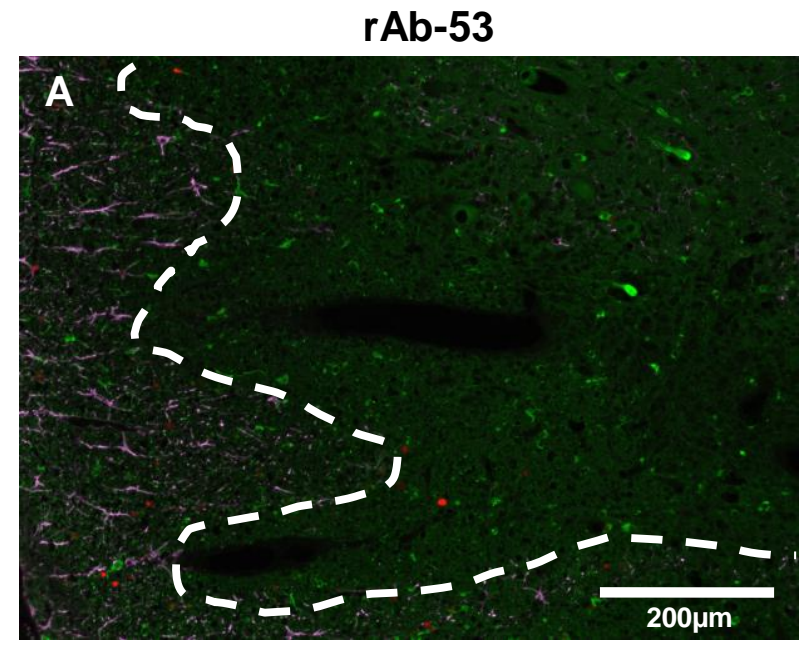

White matter lesions

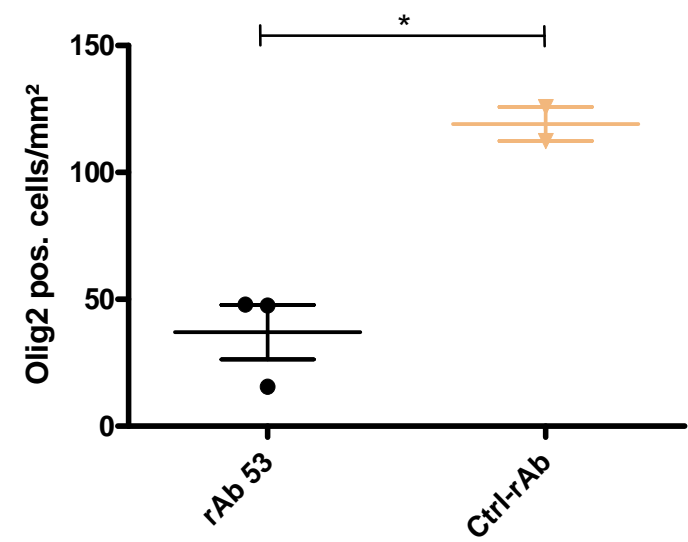

Ctrl-rAb

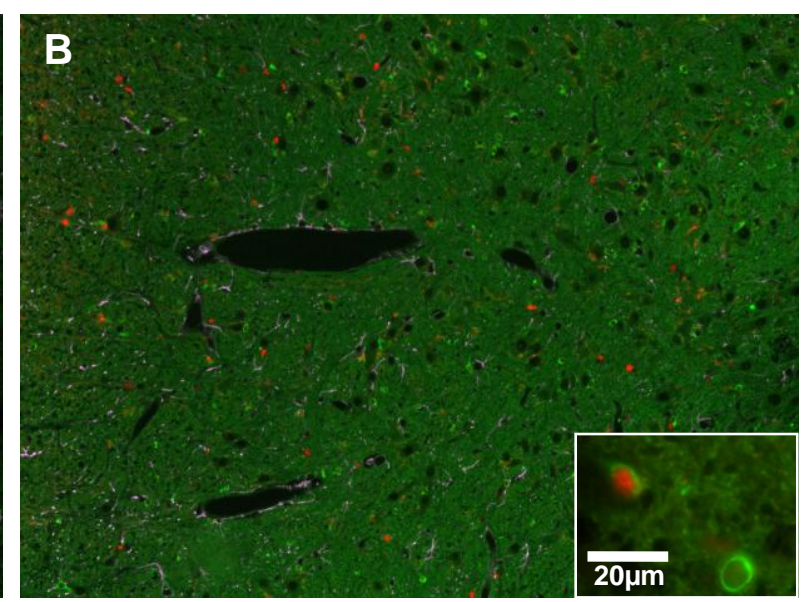

Grey matter lesions

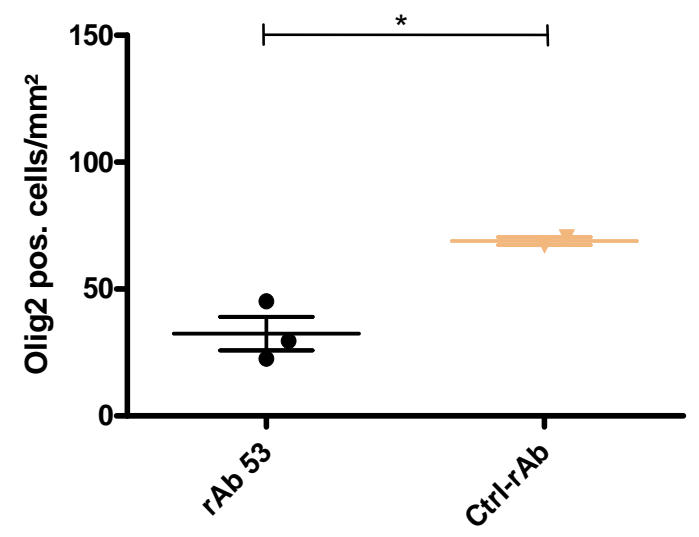

Figure 3.16: GFAP/Olig2/NogoA triple immunohistochemistry confirms the decrease of OPCs in NMO-like lesions. Spinal cord cross sections of animals i.v. injected with $r A b-53(A)$ or ctrl-rAb (B) stained for GFAP (purple), Olig2 and NogoA. Graphs demonstrate the decreased densities of OPCs in white $(P=0.0114)$ and grey matter $(P=0.0246) N M O-l i k e$ lesions compared to ctrl-rAb treated animals. Dotted line: astrocyte depleted area; small box: cell double positive for Olig2 and NogoA and singlepositive cell (NogoA).

\subsubsection{Activated caspase-3 positive oligodendrocytes in astrocyte depleted areas}

Dying oligodendrocytes were detected in astrocyte depleted areas. Part of these cells showed characteristics of apoptosis, such as cell shrinkage and nuclear fragmentation (Fig. 3.17 A+B). To determine how oligodendrocyte death occurred double stainings for NogoA and activated-caspase-3 were performed (Fig. 3.17 B). Single double-positive oligodendrocytes could be identified. Similarly, applying TUNEL staining for fragmented DNA, individual TUNEL positive oligodendrocytes were found. 

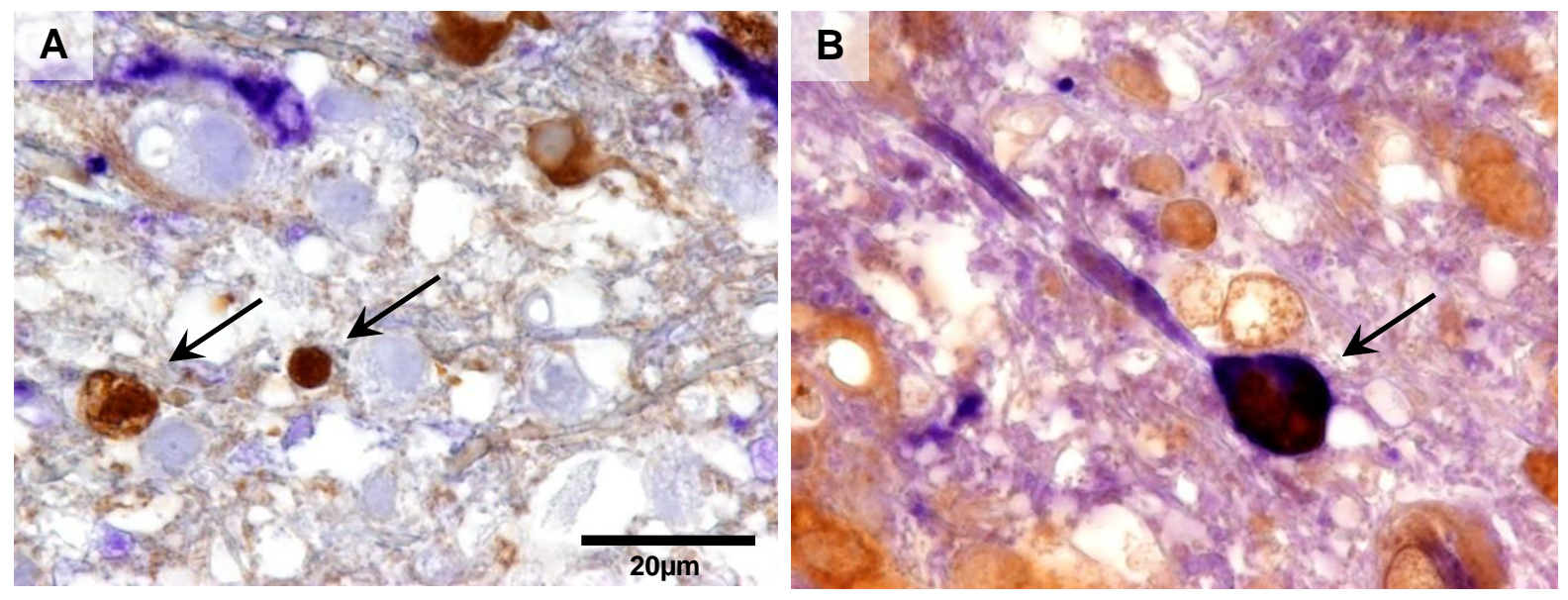

Figure 3.17: Oligodendrocyte death is caspase-3 dependent. Double IHC of NogoA (brown) and GFAP (purple) shows dying oligodendrocytes with shrunken cell bodies in NMO-like lesions (A; arrows). Double staining of NogoA (brown) and activated Caspase 3 (purple) in astrocyte depleted areas (B; arrow).

\subsubsection{Kidney pathology in the EAE/NMO model}

AQP4 is not only expressed on astrocyte foot processes, but is also a prominent water channel in the kidney. There, it is expressed at the basolateral membrane of the inner medullary collecting ducts (IMCD) (Terris et al., 1995). I was interested whether I could detect any pathological or clinical evidence for rAb-53 effects in the kidney. During the EAE/NMO experiment urine of the animals $(n=8)$ injected with recombinant rAb-53 or ctrl-rAb was collected for further analysis. 30hrs after i.v. antibody injection animals were sacrificed and kidneys processed for IHC.

AQP4 IHC of control animals showed no abnormalities in the kidney and especially the inner medulla (Fig. 3.18 A-C). However, in rAb-53 injected rats, AQP4 positive cells of IMCDs appeared swollen. Almost no space between the collecting ducts was visible any more (Fig. 3.18 D-F). Additionally some cells of IMCDs showed shrunken nuclei (Fig. 3.18 E+G-I). Cells of the collecting ducts were partly detached (Fig. 3.18 G-I). 


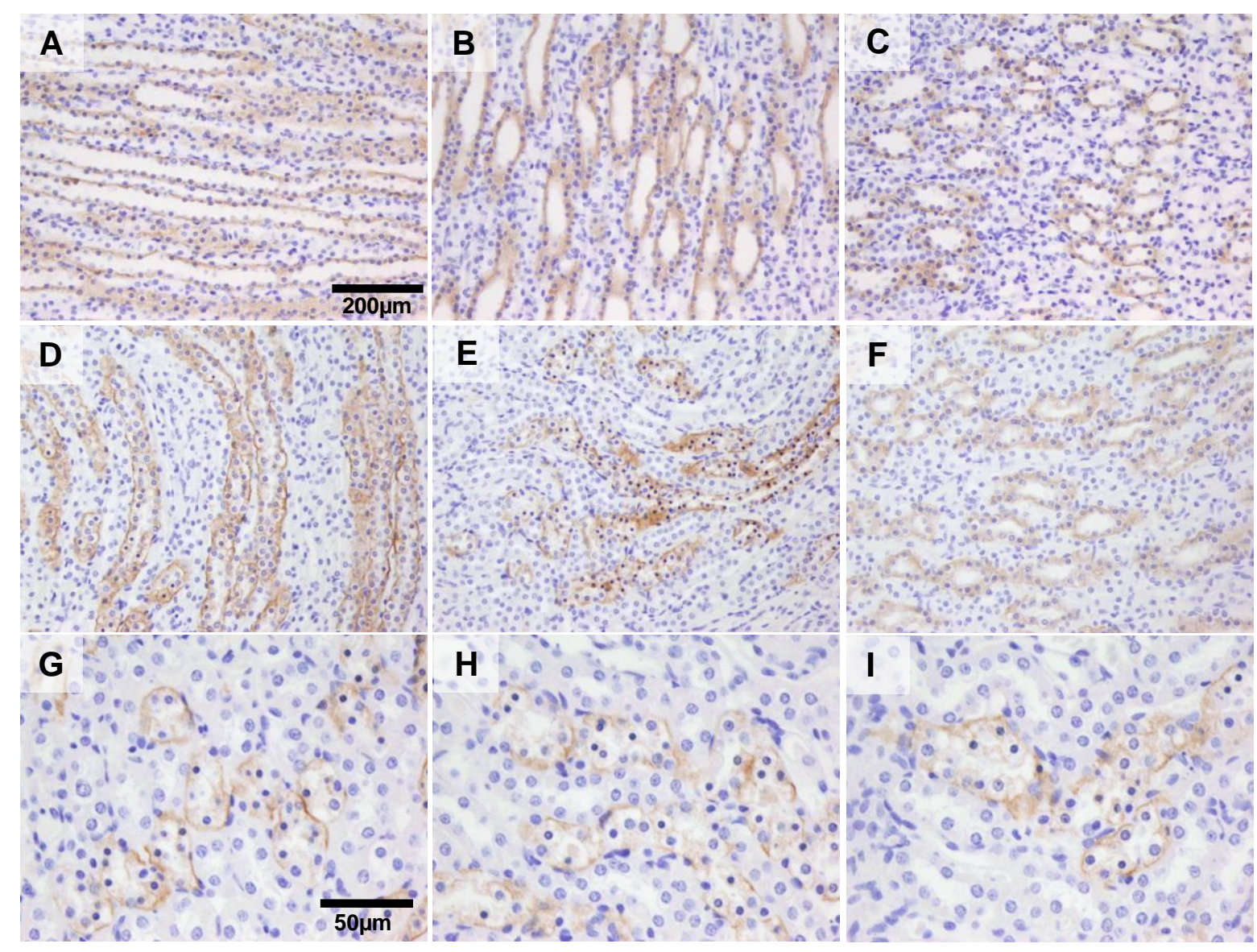

Figure 3.18: AQP4 staining (brown) of inner medullary collecting ducts (IMCDs) in the EAE/NMO model. IMCDs of animals injected with ctrl-rAb show no abnormalities (A-C). IMCD cells of animals treated with rAb-53 are swollen (D-F) and in part detached with shrunken nuclei (E+G-I).

\subsection{Macrophage infiltration in the kidneys}

Cross sections of kidneys were immunostained for ED1 to assess macrophage infiltration, most likely reflecting the phagocytosis of detached collecting duct cells. In kidneys of systemic EAE/NMO animals several foci of macrophage infiltration were found in the inner medulla (Fig. 3.19 A). In control kidneys from animals i.v. injected with ctrl-rAb only single ED1 positive cells were observed (Fig. 3.19 B). 
rAb-53

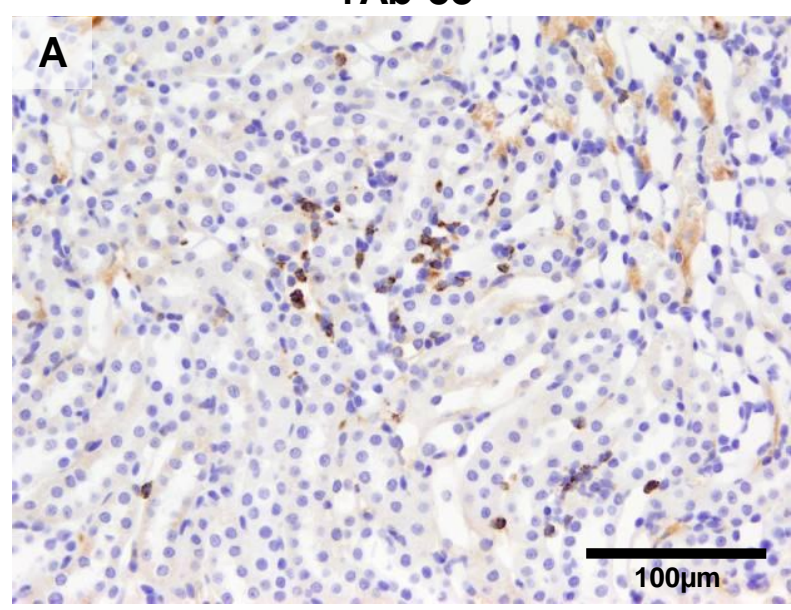

\section{Ctrl-rAb}

B

Figure 3.19: Increased macrophage infiltration in kidneys of animals injected with $\mathrm{rAb}-53$ with accumulation of ED1 positive cells (A). Animals treated with ctrl-rAb showed no accumulation of macrophages, but distinct ED1 positive cells in the kidney (B).

\subsection{Cell debris and protein content of the urine}

To assess whether cells of the collecting ducts were detached and scaled off, cytospins of urine were performed 30hrs after antibody injection. Cells were deposited on cover slips and stained for Pappenheim/Giemsa.

Figure 3.20 represents cells collected from the urine of an animal i.v. injected with recombinant human $\mathrm{rAb}-53(\mathrm{~A})$ and from the urine of a rat i.v. injected with ctrl-rAb (B). In Fig. 3.19 A the number of cells was dramatically increased compared to B.

Furthermore, in the urine of animals i.v. injected with $\mathrm{rAb}-53100 \mathrm{mg} / \mathrm{dl}$ protein was observed compared to animals injected with ctrl-rAb, where no protein was detectable after $30 \mathrm{hrs}$. 


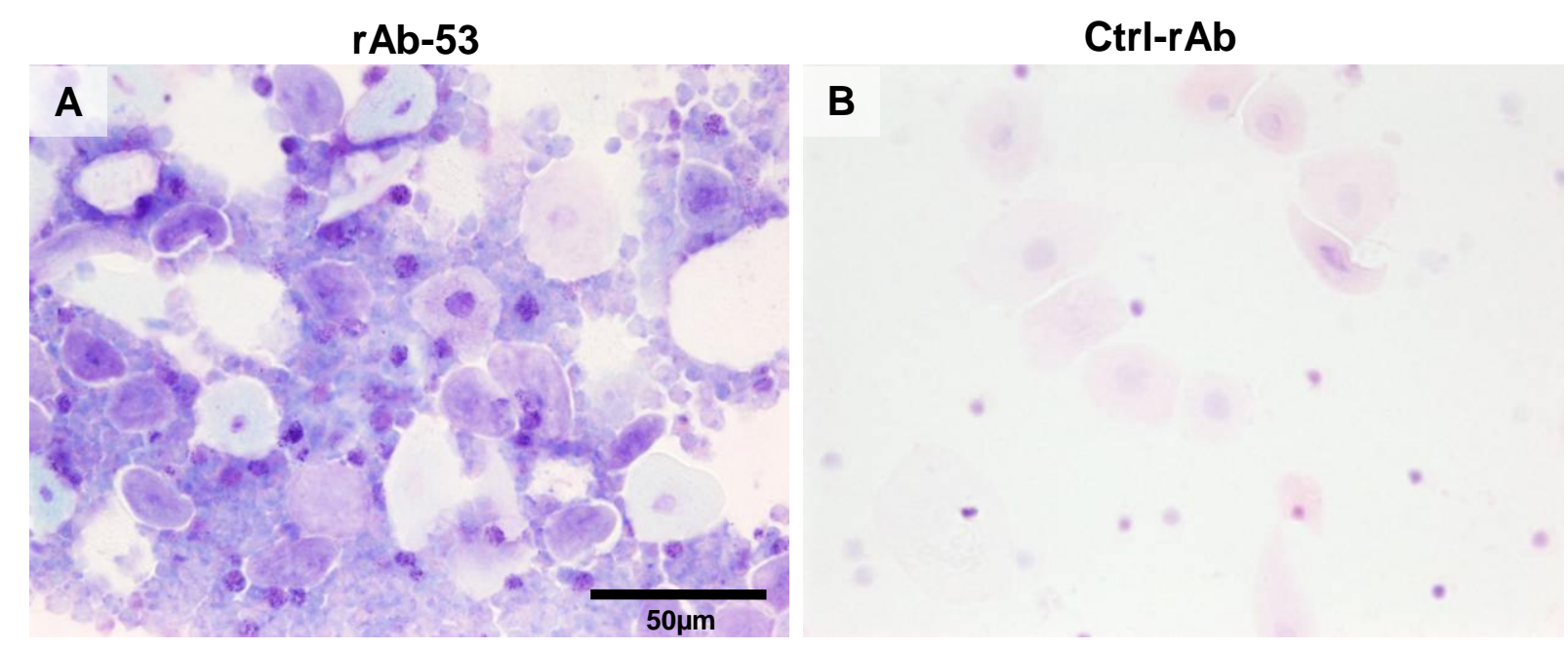

Figure 3.20: Pappenheim/Giemsa staining of a urinary cytospin collected $30 \mathrm{hrs}$ after i.v. injection of rAbs. The urinary cytospin of rats i.v. injected with rAb-53 shows dramatically increased numbers of detached IMCD cells $(A)$ in contrast to the cytospin of ctrl-rAb injected rats (B).

\subsubsection{Mutants of $\mathrm{rAb}-53$ antibodies show different extent of histopathology in the EAE/NMO model}

To determine if the ability of human rAb-53 to bind complement or act via ADCC is crucial for astrocyte depletion and lesion pathogenesis point mutated variants of $r A b$ 53 were generated and applied to my experimental system. Mutants were generated in the lab of Jeffrey Bennett, Denver, Colorado. In the systemic model of EAE/NMO rats were i.v. injected with the mutated rAb-53_K322A, rAb-53_K326W, or rAb53_L234A. K322A is unable to bind complement, K326W is defective in ADCC and L234A is defective in both.

Rats $(n=20)$ showed no significant differences in short-term (30hrs) clinical scores after i.v. injection of the above mentioned antibodies (data not shown) compared to the original rAb-53. However, by histological examination, only animals injected with the $\mathrm{rAb}-53$ showed significantly more astrocyte loss compared to animals injected with the ctrl-rAb $(P<0.005)$. Spinal cord cross sections of rats injected with the mutant antibodies showed much smaller areas of astrocyte depletion than with wt rAb-53. Thus, the ability to deplete astrocytes was substantially lower in mutants. For instance, treatment with the complement defective mutant, K322A, revealed a significant decrease in astrocyte loss compared to wt rAb-53 $(P<0.005)$ (Fig. 3.21). Additionally, a trend to decreased astrocyte loss was seen in animals injected with $\mathrm{K} 322 \mathrm{~A}$ compared to K326W, which is defective in ADCC (Fig. 3.21). 
As I mentioned before, a prominent loss of oligodendrocytes was observed when animals were i.v. injected with the rAb-53. I detected the loss of oligodendrocytes also in animals transferred with the mutant rAbs, but this loss was restricted to a much smaller area of astrocyte depletion. In white matter lesions a significant loss of NogoA positive cells was detected when animals were injected with $r A b-53(P<0.01)$ or its mutants $(P<0.001)$ compared to ctrl-rAb. Similarly, also in grey matter lesions a significant loss of oligodendrocytes was found in rats i.v. injected with $r A b-53$ or its mutants compared to ctrl-rAb $(P=0.0001)$. Densities of oligodendrocytes in astrocyte depleted areas were even lower in animals injected with one of the mutant rAb than with the wt rAb-53. However, only the complement defective mutant K322A actually showed a significant decrease in NogoA positive cells compared to rAb-53 $(P<0.005)$ (Fig. 3.22).

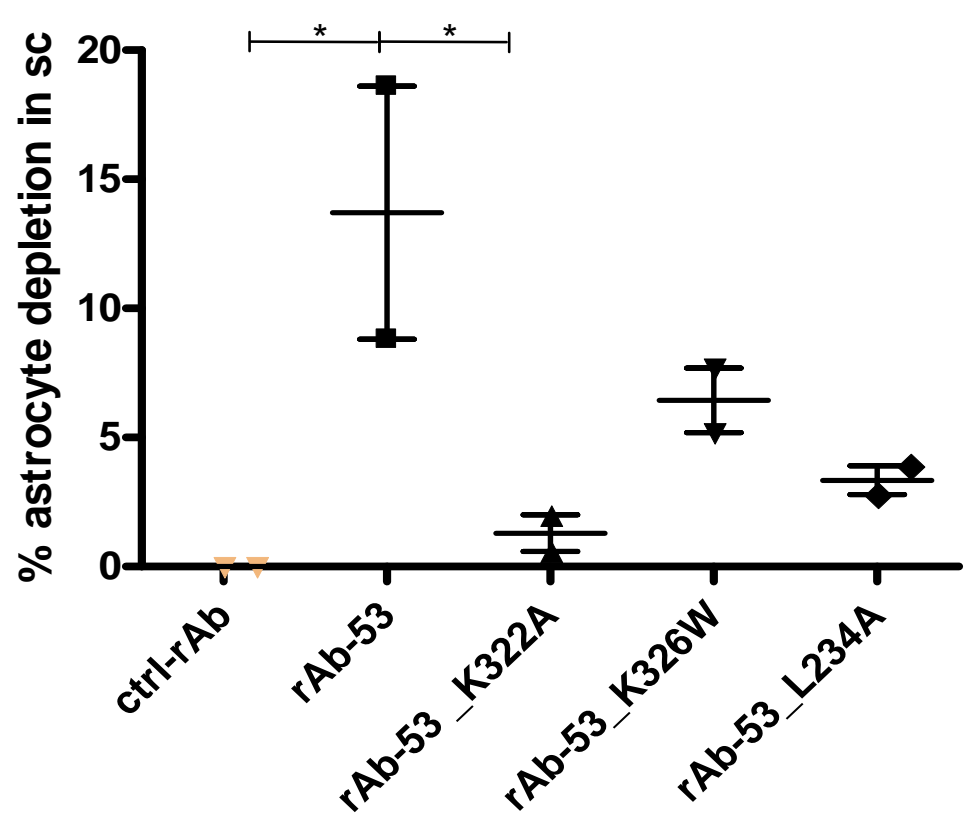

Figure 3.21: The percentage of astrocyte depleted spinal cord area is significantly higher in animals treated with $\mathrm{rAb}-53$ than with the ctrl-rAb $(P<0.05)$. Animals injected with the complement deficient mutant, K322A, show a significant decrease in astrocyte depletion compared to wt $\mathrm{rAb}-53$ $(\mathrm{P}<0.05)$. There is no significant decrease in astrocyte depletion with the mutants K326W and L234A compared to wt rAb-53. 

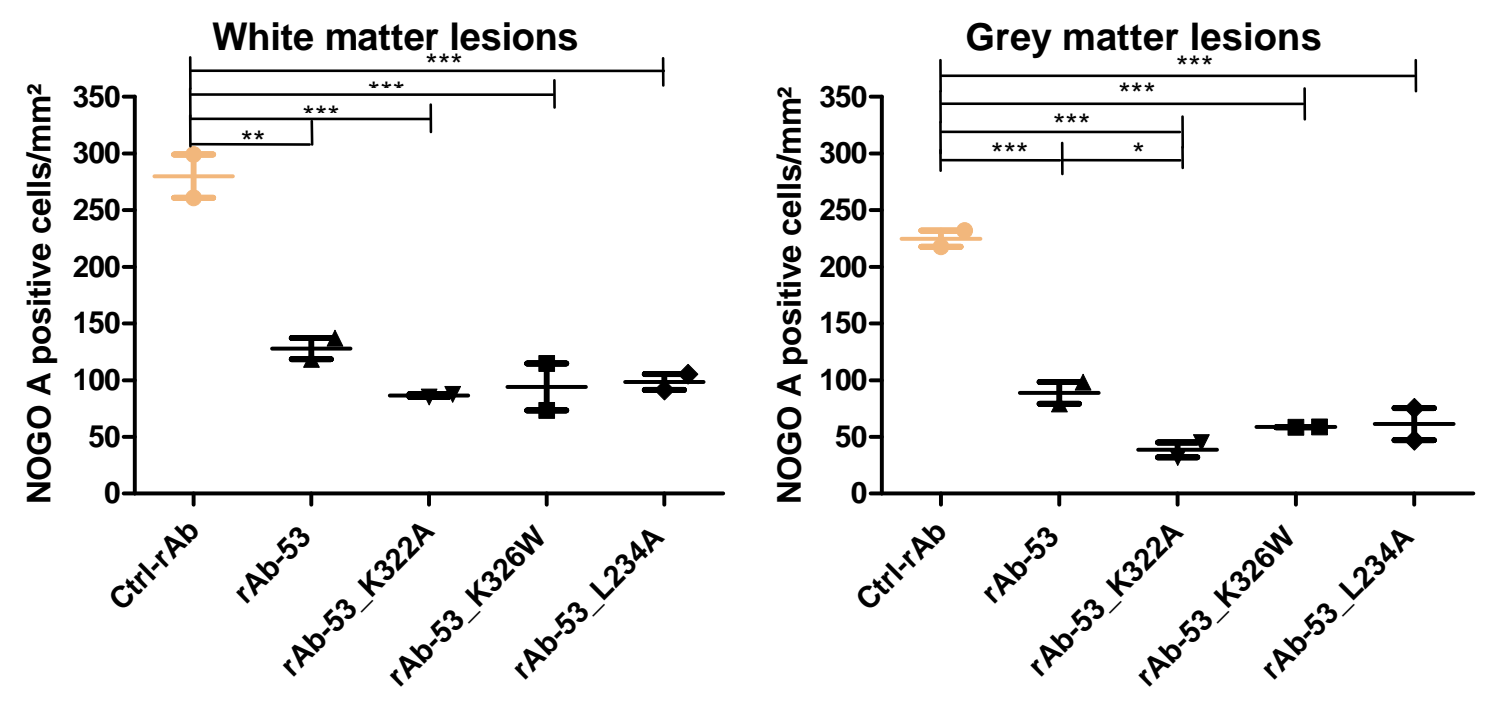

Figure 3.22: Numbers of mature oligodendrocytes in EAE/NMO in white and grey matter lesions. Animals were injected with $\mathrm{rAb}-53$ or corresponding mutants deficient in complement binding, ADCC activation, or both. In white matter lesions a significant loss of mature oligodendrocytes was detected in animals treated with rAb-53 $(P<0.01)$ or its mutants $(P<0.001)$. A significant loss of NogoA positive cells was also visible in the grey matter when animals were injected with $\mathrm{rAb}-53$ or its mutants. The cell numbers were even lower in mutants than in rAb-53 injected cases. Even injection of the mutant K322A showed a significant decrease of oligodendrocytes compared to $\mathrm{rAb}-53$.

\subsubsection{Effect of $\mathrm{rAb}-53$ on astrocytes and oligodendrocytes in vitro}

In vivo, no clear-cut effect of NMDA and $\mathrm{P}_{2} \mathrm{X}_{7}$ receptor antagonists on oligodendrocyte death was found in the focal NMO model. To further test whether oligodendrocyte death was related to astrocyte death in vitro experiments $(n=3)$ were performed. Astrocytes and oligodendrocytes were cultured from newborn rats.

In a first approach astrocytes were incubated in medium, in medium supplemented with rAb-53 $(1 \mu \mathrm{g} / 200 \mu \mathrm{l})$ alone, with human serum alone or with rAb-53 $(1 \mu \mathrm{g} / 200 \mu \mathrm{l})$ and human serum for $24 \mathrm{hrs}$ followed by PFA fixation.

Medium and $\mathrm{rAb}-53$ only treated astrocytes (Fig. 3.23. A+B) grew densely as visualized by DAPI/GFAP/O4 staining and looked healthy. This was supported by some $\mathrm{O} 4$ positive OPCs which only settle on intact astrocyte cell layers. A decrease in astrocyte density was observed in cultures treated with human serum alone. However, still some $\mathrm{O} 4$ positive cells were attached to astrocytes (Fig. 3.23 C). When astrocytes were exposed to $\mathrm{rAb}-53$ together with human serum cell layers were dissolved. GFAP positive astrocyte processes had disappeared. Only DAPI and GFAP positive shrunken cells were visible. No O4 positive cells were detected any more (Fig. $3.23 \mathrm{C}$ ) 
To underpin the microscopic findings WST-1 cell viability and LDH cytotoxicity assays of astrocyte cultures were performed. The WST-1 assay did not show any differences in proliferation and viability under different treatment conditions (data not shown). The LDH assay indicated more cell loss in astrocyte cultures treated with rAb-53 with human serum than incubated with medium or rAb-53 alone (Fig. 3.24). Additionally, cytoxicity was also increased after incubation with human serum alone (Fig. 3.24).
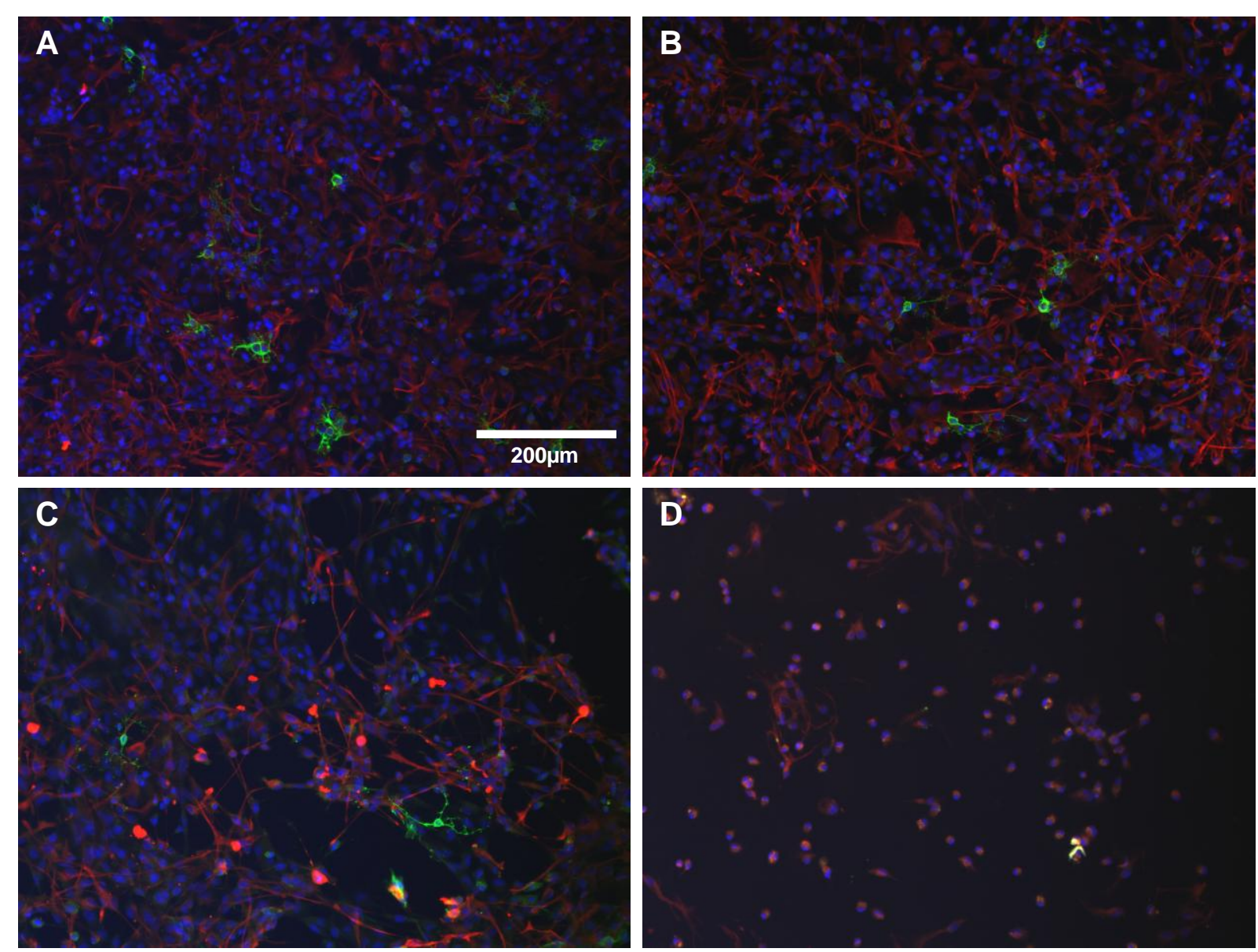

Figure 3.23: Astrocyte cell culture stained for DAPI (blue), GFAP and O4. Cells were incubated for 24hrs with medium (A), with rAb-53 alone (B), with human serum alone $(C)$ or with rAb-53 together with human serum (D), which causes reduction of GFAP positive cells and processes. 


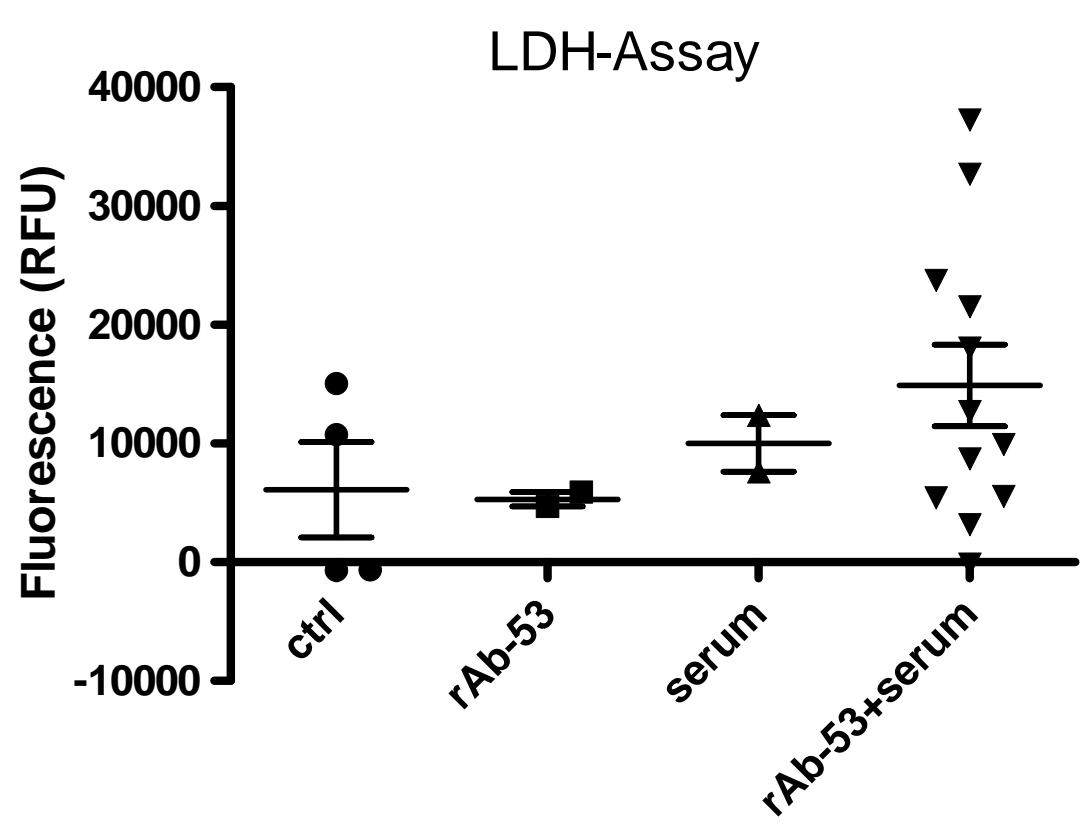

Figure 3.24: LDH (cytotoxicity) assay of representative astrocyte cultures incubated in medium alone, in medium supplemented with the rAb-53 alone, with human serum alone or with $\mathrm{rAb}-53$ and human serum. RFU: Relative Fluorescence Units.

In a second approach oligodendrocyte cultures were incubated with medium, with medium supplemented with $\mathrm{rAb}-53$ alone $(1 \mu \mathrm{g} / 200 \mu \mathrm{l})$, with human serum alone, with rAb-53 $(1 \mu \mathrm{g} / 200 \mu \mathrm{l})$ together with human serum, or with supernatants collected from astrocyte cultures incubated with $\mathrm{rAb}-53$ and human serum. The treatment was applied for 24hrs followed by PFA fixation.

Oligodendrocyte cultures incubated in medium alone were densely populated and immunopositive for $\mathrm{O} 4$ and in part MBP (Fig. 3.25 A). The same was true for oligodendrocytes treated with rAb-53 alone (Fig. 3.25 B). When cultures were treated with human serum alone, the cell density was not decreased and cells were still positive for $\mathrm{O} 4$ and MBP. Some oligodendrocytes appeared swollen (Fig. 3.25 C). Incubation with the $\mathrm{rAb}-53$ together with human serum similarly did not decrease oligodendrocyte density (Fig. 3.25 D). After the incubation with the supernatant from astrocytes incubated with $\mathrm{rAb}-53$ and human serum, oligodendrocyte density appeared diminished and oligodendrocyte processes were in part fragmented (Fig. $3.25 \mathrm{E}+\mathrm{F})$. 

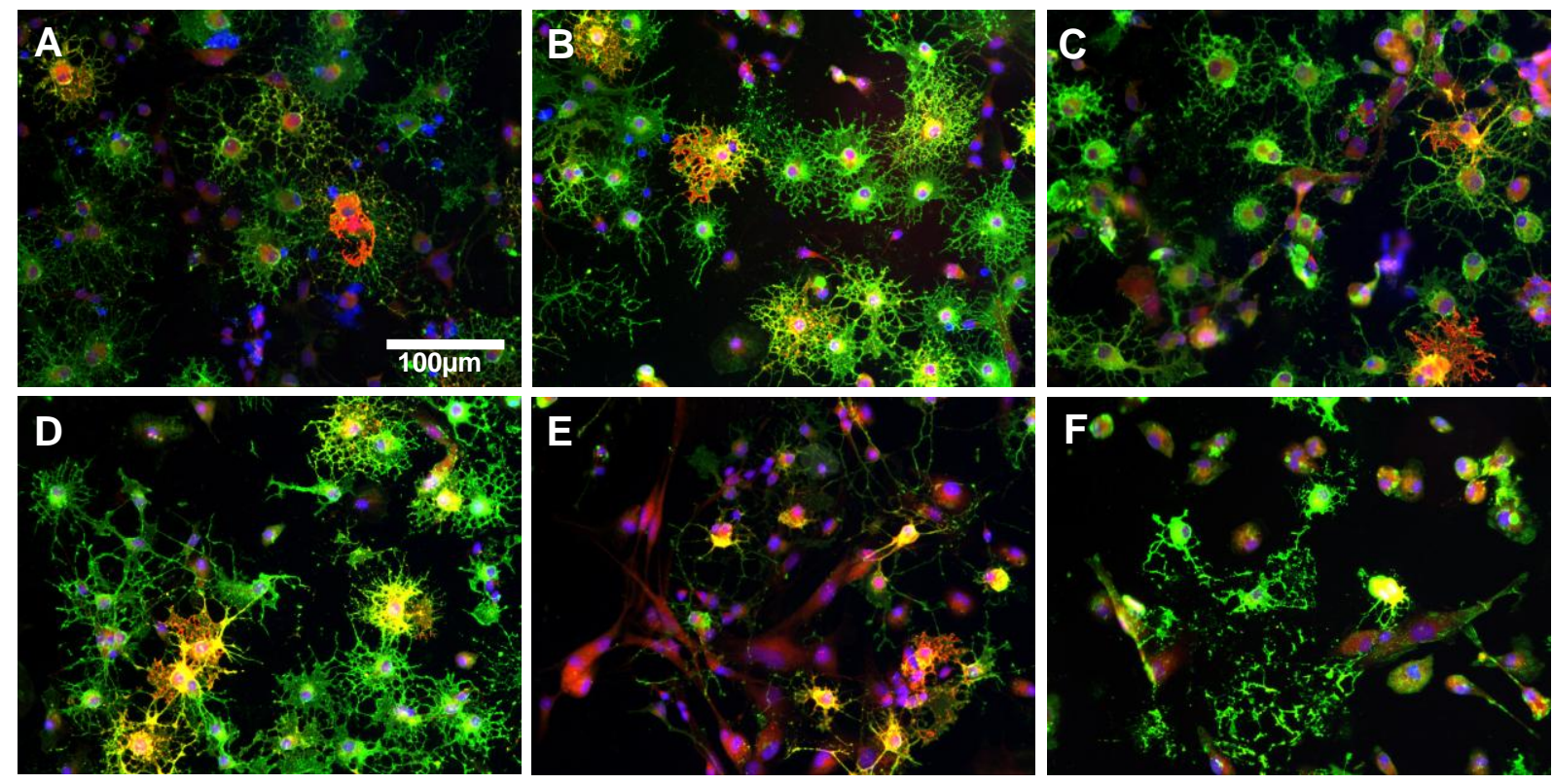

Figure 3.25: Oligodendrocyte cell cultures stained with DAPI (blue), MBP and O4. Cells were treated for $24 \mathrm{hrs}$ with medium (A), rAb-53 alone (B), human serum (C), rAb-53 together with human serum (D) or supernatants of $r A b-53 /$ human serum treated astrocytes $(E+F)$, which induced a reduction in oligodendroglia.

\subsubsection{Assessment of mechanisms of oligodendroglial cell death in vitro}

\subsection{NMDA and $P_{2} X_{7}$ receptor antagonists}

To investigate whether NMDA and $\mathrm{P}_{2} \mathrm{X}_{7}$ receptor antagonists prevent oligodendrocyte death in vitro, cultures were incubated with supernatants from astrocytes treated with $\mathrm{rAb}-53$ and human serum. This treatment resulted in a decrease in oligodendrocytes (Fig. 3.25). In an attempt to rescue these cells one of the receptor antagonists was added. First, the receptor antagonists $(50 \mu \mathrm{M})$ were added to untreated astrocyte and oligodendrocyte cultures. No effects on cell density and cell morphology were observed. After treatment of oligodendrocyte cultures incubated with supernatants of astrocyte cultures, incubated before firstwith $r A b-53$ in human serum, with NMDA or $\mathrm{P}_{2} \mathrm{X}_{7}$ receptor antagonists $(50 \mu \mathrm{M})$, first microscopic analysis did not show any decrease in oligodendrocyte death. Also the WST-1 assay did not show any differences in cell viability (data not shown). 


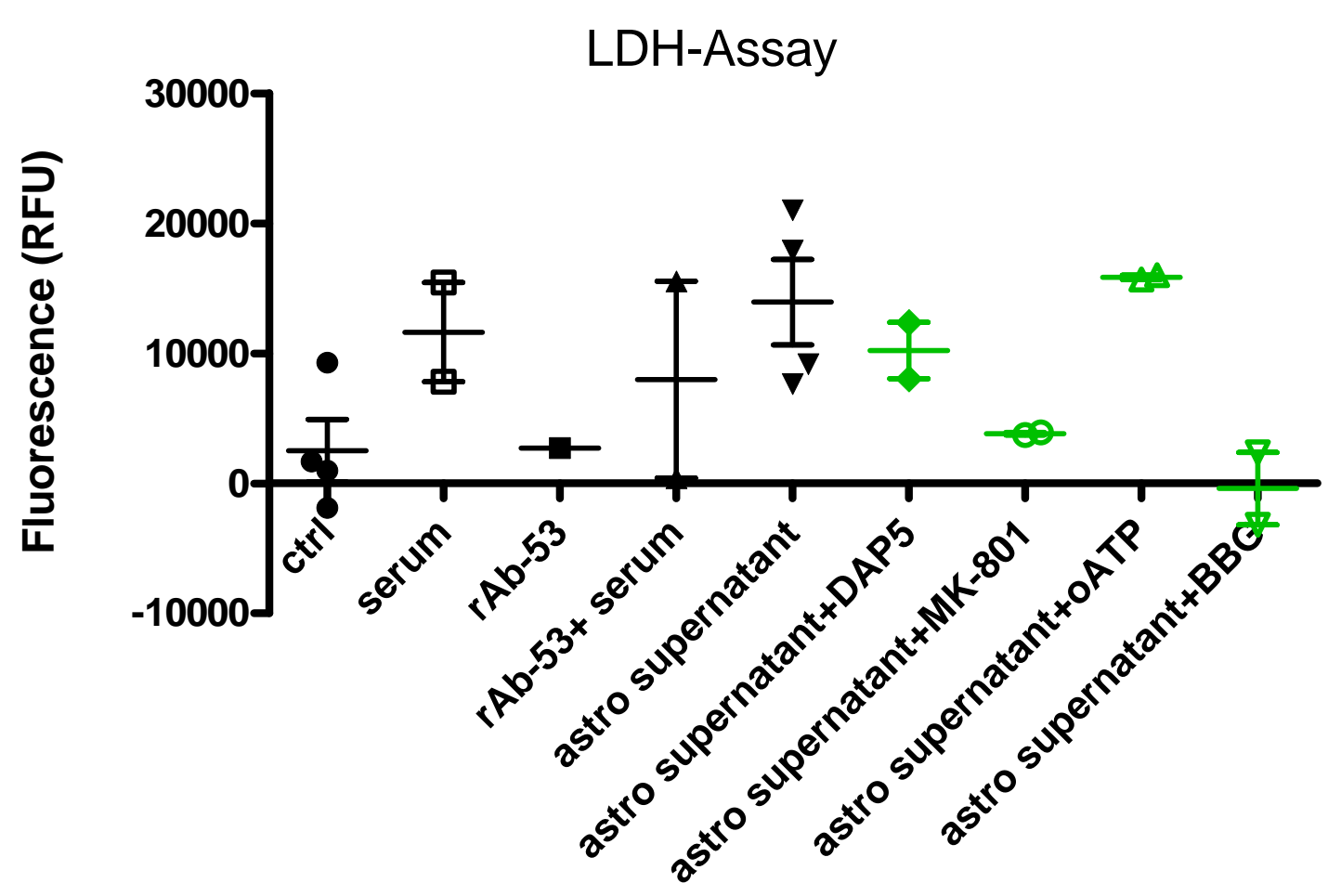

Figure 3.26: LDH (cytotoxicity) assay of representative oligodendrocyte cultures untreated, treated with human serum alone, with $\mathrm{rAb}-53$ alone, with $\mathrm{rAb}-53$ + human serum, with astrocyte culture supernatant (astrocytes previously incubated in $\mathrm{rAb}-53$ and human serum), astrocyte culture supernatant + D-AP5, astrocyte culture supernatant + MK-801, astrocyte culture supernatant + OATP or astrocyte culture supernatant + BBG. RFU: Relative Fluorescence Units

The analysis of the LDH assay revealed an increase in cytotoxicity when oligodendrocytes were treated with supernatants from astrocytes incubated with rAb53 in human serum (Fig. 3.26). However, similar to my findings in the astrocyte culture system toxicity was already increased by human serum alone. Of note, the range of $\mathrm{LDH}$ release was similar in controls and cultures treated with rAb-53 alone. The LDH assay indicated a decrease in oligodendrocyte toxicity after incubation with supernatant from treated astrocytes when MK-801 $(50 \mu \mathrm{M})$ or BBG $(50 \mu \mathrm{M})$ were added (Fig. 3.26). 


\section{PART 2}

\subsection{Effect of astrocytic overexpression of FGF9}

Recent in vitro findings indicated a possible role of FGF9 on DM and RM. I wanted to test whether this is true in my in vivo models of DM and RM. First, I applied FGF9 protein to demyelinated lesions in cuprizone-fed animals via stereotactic injection. However, most likely because of rapid degradation and the need for a prolonged availability of FGF9, no effect on RM was observed. To provide continuous and sufficient overexpression of this growth factor in vivo, an adeno-associated virus 6 (AAV-6)-based vector containing the cDNA of FGF9 under the control of the GFAP promotor was constructed (FGF9-AAV-6-based vector). AAV-6-based vector predominantly infects astrocytes. In addition, the GFAP-driven construct conveys expression specifically in astrocytes. Control animals were injected with AAV-6 expressing EGFP under control of the GFAP promoter (EGFP-AAV-6-based vector). Previous experiments showed the highest FGF9 (Fig. 3.27 A) and EGFP (Fig. 3.27 D) expression 7 to 10 days after intracerebral AAV-6-based vector injection. To assess the role of FGF9 on myelin formation in vivo two experimental models of deand RM were used. 


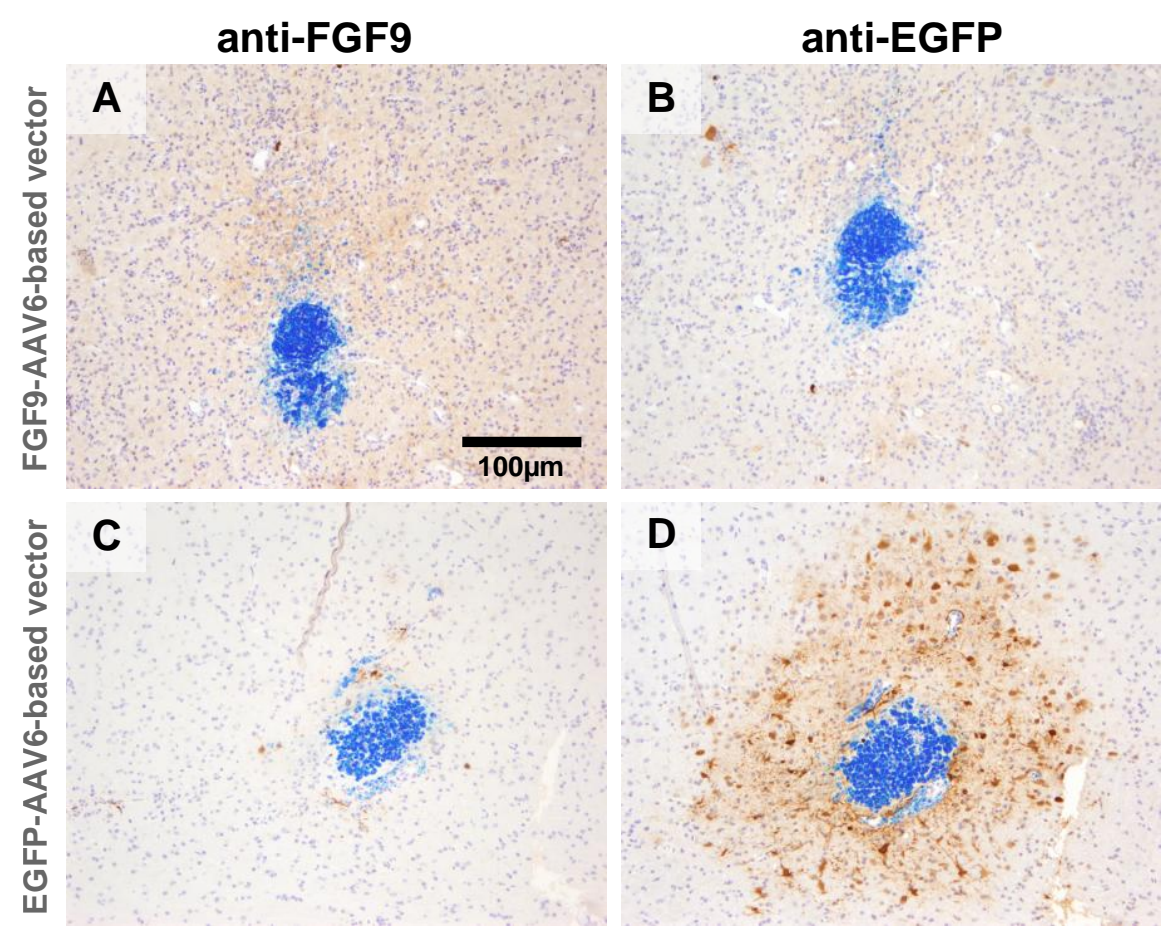

Figure 3.27: Expression of FGF9 and EGFP on day 10. Animals intracerebrally injected with FGF9AAV-6-based vector reveal a diffuse expression of FGF9 at day 10 (A; FGF9), whereas animals treated with EGFP-AAV-6-based vector are negative for FGF9 (C; FGF9). In contrast, EGFP staining is negative in animals injected with FGF9-AAV-6-based vector (B; EGFP), however EGFP-AAV-6based vector treated animals show positive cellular EGFP staining (D; EGFP).

\subsubsection{Astrocyte and oligodendroglial phenotype after intracerebral} FGF9-AAV-6-based vector injection in the cuprizone mouse model

Mice $(n=7)$ were fed with $0.25 \%$ cuprizone for 5 weeks. At week 4 animals were injected with FGF9-AAV-6-based vector $(2 \mu \mathrm{l})$, an EGFP-AAV-6-based control vector, or PBS. Half of the mice were sacrificed after 7 days to study the influence of FGF9 on the extent of DM. The others were sacrificed after 10 days including 3 days of normal chow to study the influence of FGF9 expression on RM.

After 5 weeks on cuprizone diet DM was near complete in the corpus callosum and cortex (Fig. 3.28 A, B, E, F, I, J). In one animal injected with FGF9-AAV-6-based vector, the demyelinated area was larger than usual (Fig. $3.28 \mathrm{I}+\mathrm{J}$ ). However, as a whole, FGF9 did not alter the extent of demylination in the cuprizone model 7 days after injection. But astrocytes were swollen, their cytoplasms were enlarged and their processes were well visible, representing a strongly activated phenotype at this time point (Fig. $3.28 \mathrm{~K}$ ). This was not seen in mice injected with PBS or EGFP-AAV-6based vector (Fig. 3.28 $\mathrm{C}+\mathrm{G}$ ). The number of proliferating, Ki67 positive cells as well as the number of mitotic figures was much higher after injection of the FGF9-AAV-6- 
based vector (Fig. 3.28 L) than with PBS or the EGFP-AAV-6-based vector (Fig. 3.28 $\mathrm{D}+\mathrm{H})$.

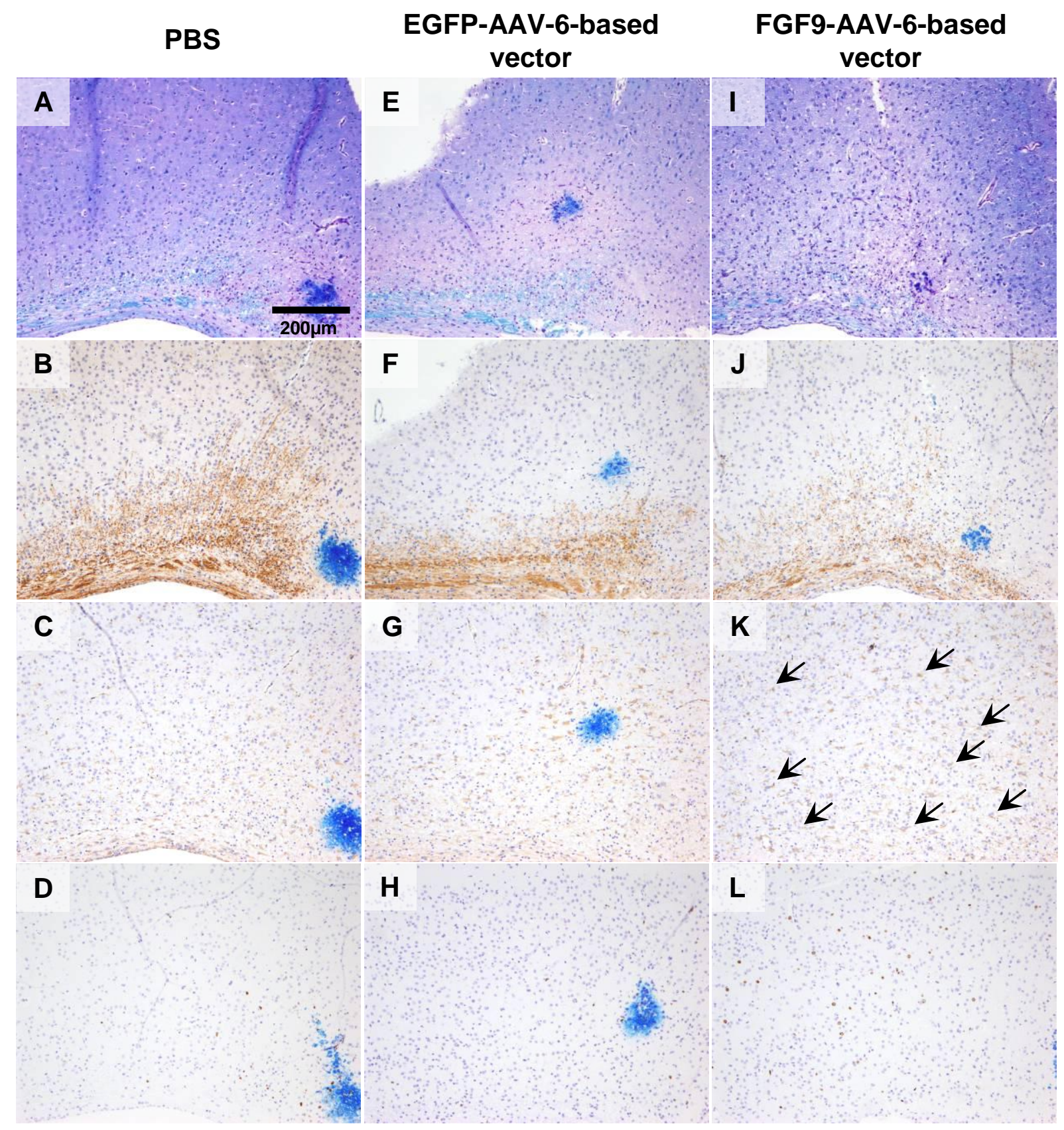

Figure 3.28: Hemispheric frontal brain sections of mice injected with PBS (A-D), EGFP-AAV-6based vector (E-H) or FGF9-AAV-6-based vector (I-L) in the cuprizone model. Mice were perfused 7 days post injection after 5 weeks of cuprizone feeding. The demyelinated area appears the same size in animals treated with FGF9-AAV-6-based vector (I, J) in LFB-PAS (A, E, I) and MBP (B, F, J) IHC. GFAP (C, G, K) positive astrocytes show a strongly activated phenotype in animals injected with FGF9-AAV-6-based vector (K, arrows). Numbers of proliferating cells in Ki67 staining (D, $H, L)$ were increased in animals treated with FGF9-AAV-6-based vector (L).

The numbers of mature oligodendrocytes were increased in FGF9-AAV-6-based vector treated animals (Fig. 3.29 F). Also, the numbers of Olig2 positive OPCs were markedly increased in animals injected with FGF9-AAV-6-based vector (Fig. 3.29 E), 
but not in controls (Fig. 3.29 $\mathrm{A}+\mathrm{C}$ ). Morphologically, Olig2 positive cells were markedly enlarged, as a whole representing an activated phenotype in FGF9-AAV-6based vector treated mice. Additionally, mitotic Olig2 positive cells were detected (Fig. 3.29 E, small box).

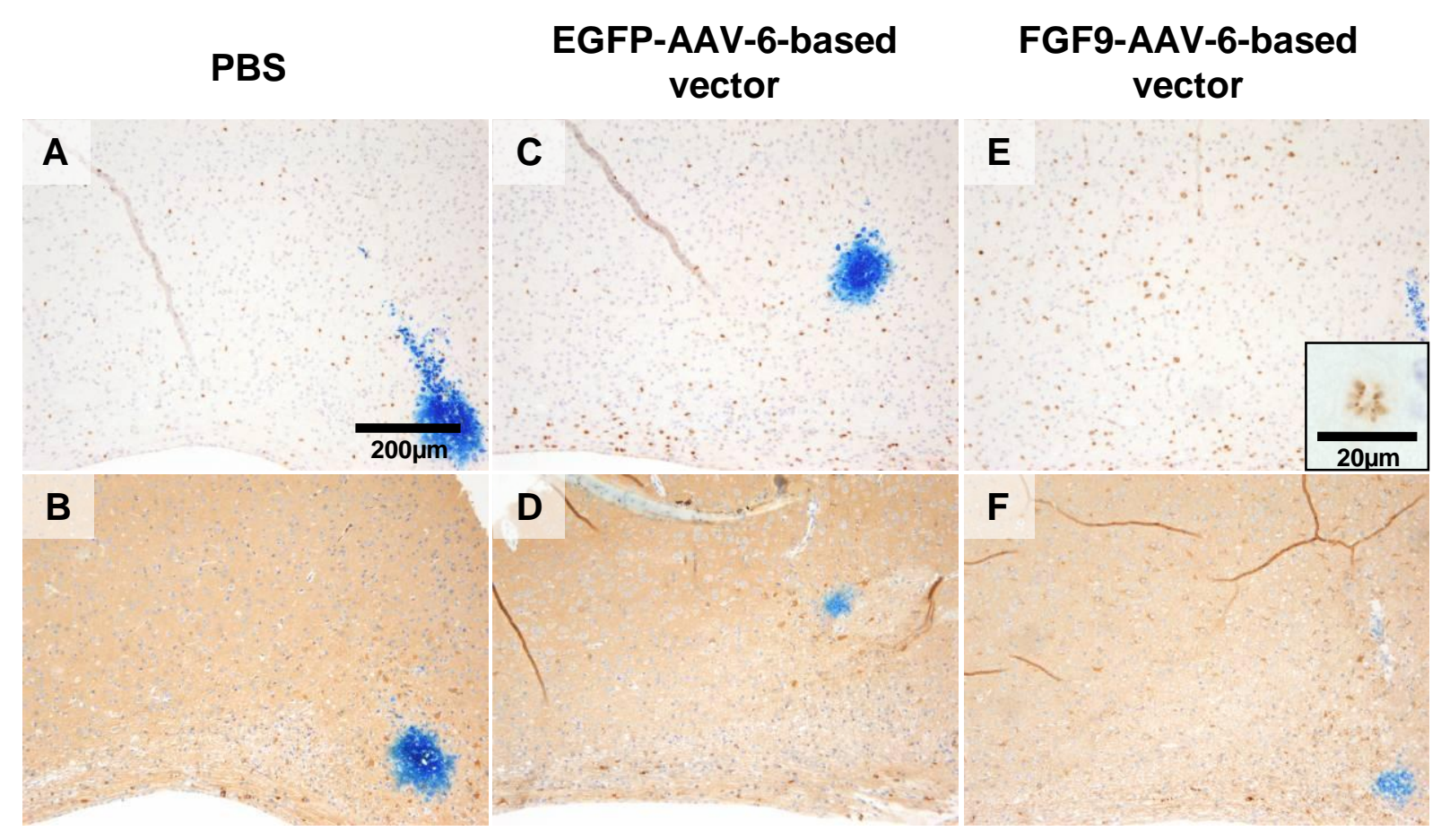

Figure 3.29: Hemispheric frontal brain sections of mice injected with PBS, EGFP-AAV-6-based vector (C-D) or FGF9-AAV-6-based vector (E-F) in the cuprizone model. Mice were perfused 7 days post injection. Olig2 (A, C, E) and $\operatorname{NogoA}(B, D, F)$ IHC showed higher cell numbers in animals treated with the FGF9-AAV-6-based vector. Small box: mitotic Olig2 positive cell.

When animals were perfused 10 days post injection, thus including 3 days of RM, no differences in the extent of RM between the experimental groups were detected (Fig. 3.30 A, B, E, F, I, J). As already demonstrated on day 7 , astrocytes and their nuclei were large, their processes were easily to be discerned, and thus showed a highly activated phenotype (Fig. $3.30 \mathrm{~K}$ ). Activated astrocytes were more abundant than 7 days post injection and were found all over the ipsilateral injected hemisphere. Also 10 days after injection with the FGF9-AAV-6-based vector the numbers of Ki67 positive cells were increased (Fig. $3.30 \mathrm{~L}$ ) compared to the numbers of proliferating cells in mice injected with PBS or EGFP-AAV-6-based vector (Fig. 3.30 D+H). Similar to the findings on day 7 Olig2 positive cells were large and appeared activated in FGF9-AAV-6-based vector treated animals. The density of Olig2 positive cells was even higher than on day 7 and mitotic figures were apparent (Fig. 3.31 E; small box). Similarly, in NogoA IHC numbers of activated mature oligodendrocytes were higher 
in mice injected with FGF9-AAV-6-based vector 10 days than 7 days after (Fig. 3.31 F). The density of mature oligodendrocytes was much lower in mice injected with PBS or EGFP-AAV-6-based vector (Fig. 3.31 B+D).

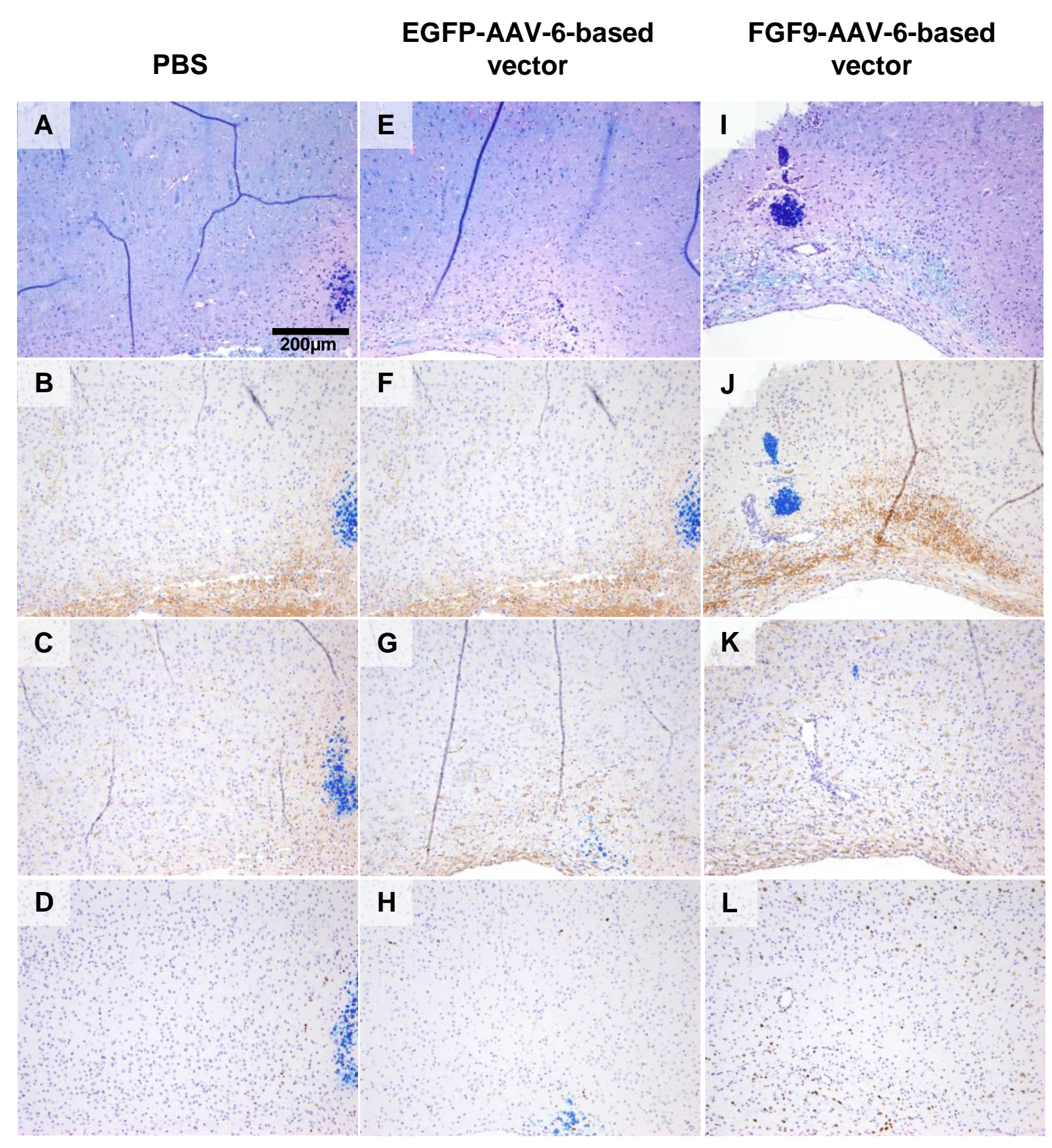

Figure 3.30: Hemispheric frontal brain sections of mice injected with PBS (A-D), EGFP-AAV-6based vector (E-H) or FGF9-AAV-6-based vector (I-L) in the cuprizone model. Mice were perfused 10 days post injection. Cuprizone diet was terminated 3 days before perfusion. No differences were detected in the extent of DM, as visualized by LFB-PAS $(A, E, I)$ and MBP $(B, F, J)$ staining. GFAP (C, G, K) positive cells showed swollen cell bodies and easily visible processes indicating a highly activated phenotype in animals treated with FGF9-AAV-6-based vector (K). Numbers of proliferating cells (Ki67) (D, H, L) were markedly increased in animals treated with FGF9AAV-6-based vector (L). 


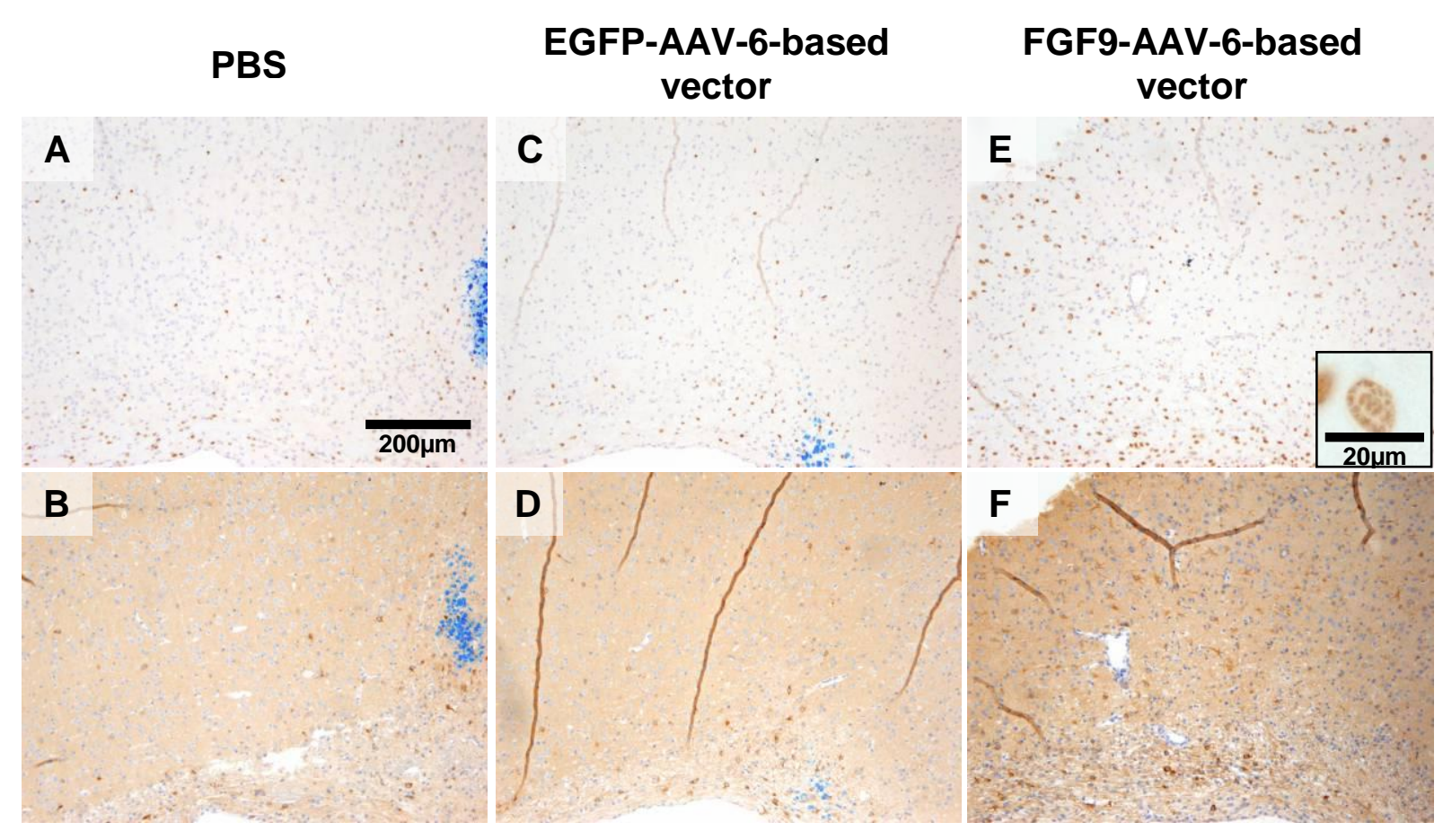

Figure 3.31: Brain sections of mice injected with PBS, EGFP-AAV-6-based vector (C-D) or FGF9-AAV-6-based vector (E-F) in the cuprizone model. Mice were perfused 10 days post injection. Olig2 $(A, C, E)$ and NogoA $(B, D, F)$ stainings showed higher cell numbers in animals treated with the FGF9-AAV-6-based vector. Small box: mitotic Olig2 positive cell.

\subsection{2}

Astrocyte and oligodendroglial phenotype after intracerebral FGF9-AAV-6-based vector injection in focal EAE

FGF9 overexpression in the cuprizone model resulted in astrocyte and oligodendroglial proliferation and activation. However, no effect on the velocity and extent of RM was observed. To assess the impact of FGF9 overexpression in a model of antibody-mediated DM in the rat, the targeted cerebral EAE model developed in our lab was utilized (Merkler et al., 2006). Female Lewis rats ( $n=39$ ) were immunized with $\mathrm{MOG}_{1-125}$ protein. After around 18 days, when the rats obtained stable anti-MOG antibody titers, they were intracerebrally injected with cytokines (TNF- $\alpha$ and INF- $\gamma$ ). The largest area of DM was visible at day 3, and complete RM occurred within 14-20 days.

Injection (2 $\mu \mathrm{l}$ ) of EGFP- (Fig. 3.32 A) or FGF9-AAV-6-based vector (Fig. 3.32 C) into the cortex of naive rats did not reveal any alterations in myelin staining 10 days after injection. However, GFAP staining revealed a panhemispheric activated phenotype of astrocytes in rats injected with FGF9-AAV-6-based vector (Fig. 3.32 D) compared to EGFP-AAV-6-based vector (Fig. 3.32 B). 
Then, $\mathrm{MOG}_{1-125}$ immunized rats were intracerebrally injected $(2 \mu \mathrm{l})$ with cytokines, to induce a focal demyelinated lesion, cytokines with EGFP-AAV-6-based vector or cytokines with FGF9-AAV-6-based vector. Unexpectedly, no DM was observed in all experimental groups 3 days after injection (Fig. 3.33 A, C, E). No differences were observed in the numbers of OPCs and mature oligodendrocytes at this time point. The numbers of Ki67 and ED1 positive cells were also comparable and low in all 3 experimental groups at day 3 .
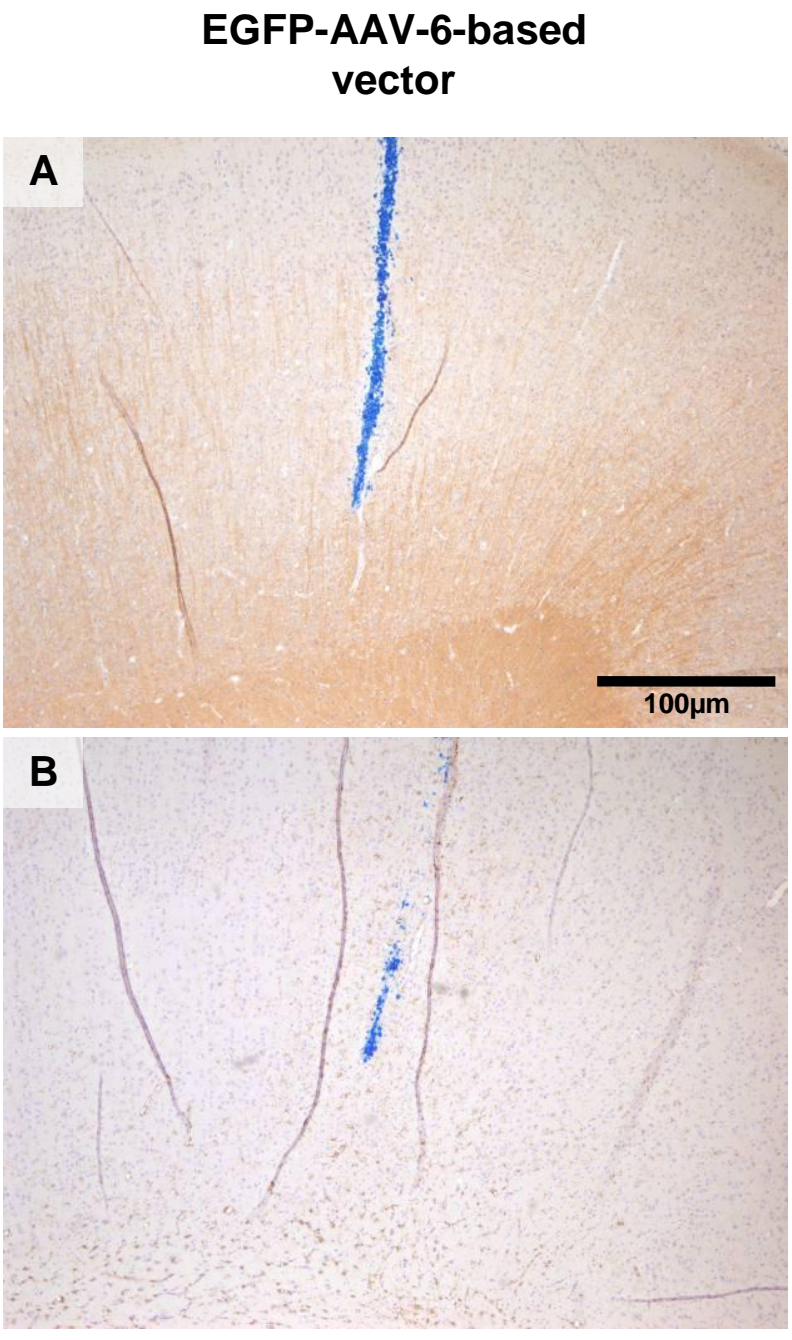

FGF9-AAV-6-based vector

\section{C}

D

Figure 3.32: 10 days after EGFP-AAV-6-based vector (A, B) or FGF9-AAV-6-based vector (C, D) injections in naïve rats. No DM (C; MBP), but strong activation of astrocytes with FGF9-AAV-6based vector is present (D; GFAP). Animals treated with EGFP-AAV-6-based vector show no DM (A; $\mathrm{MBP}$ ) and only perilesional astrocyte activation (B; GFAP).

Ten days after intracerebral injections in the rat focal EAE model small foci of demylination were only detected in rats injected with cytokines together with FGF9AAV-6-based vector (Fig. $3.34 \mathrm{M}$ ). In contrast, the myelin of rats injected with cytokines alone and cytokines with FGF9-AAV-6-based vector was well preserved 
(Fig. 3.34 $\mathrm{A}+\mathrm{G}$ ). However, at this time point, an important near panhemispheric activation of astrocytes was detected in animals injected with cytokines and FGF9AAV-6-based vector (Fig. $3.34 \mathrm{~N}$ ). This was not observed in rats injected with cytokines alone or cytokines with EGFP-AAV-6-based vector (Fig. $3.34 \mathrm{~B}+\mathrm{H}$ ). Additionally, an increase in Olig2 and NogoA positive cells was obvious in rats injected with cytokines and FGF9-AAV-6-based vector after 10 days (Fig. 3.34 O+P). Furthermore, numbers of ED1 positive cells appeared higher and more widespread (Fig. 3.34 Q) than in rats injected with cytokines alone (Fig. 3.34 E) or cytokines with EGFP-AAV-6-based vector (Fig. 3.34 K). The numbers of Ki67 positive cells were again increased in rats injected with cytokines with FGF9-AAV-6-based vector (Fig. $3.34 \mathrm{R})$.

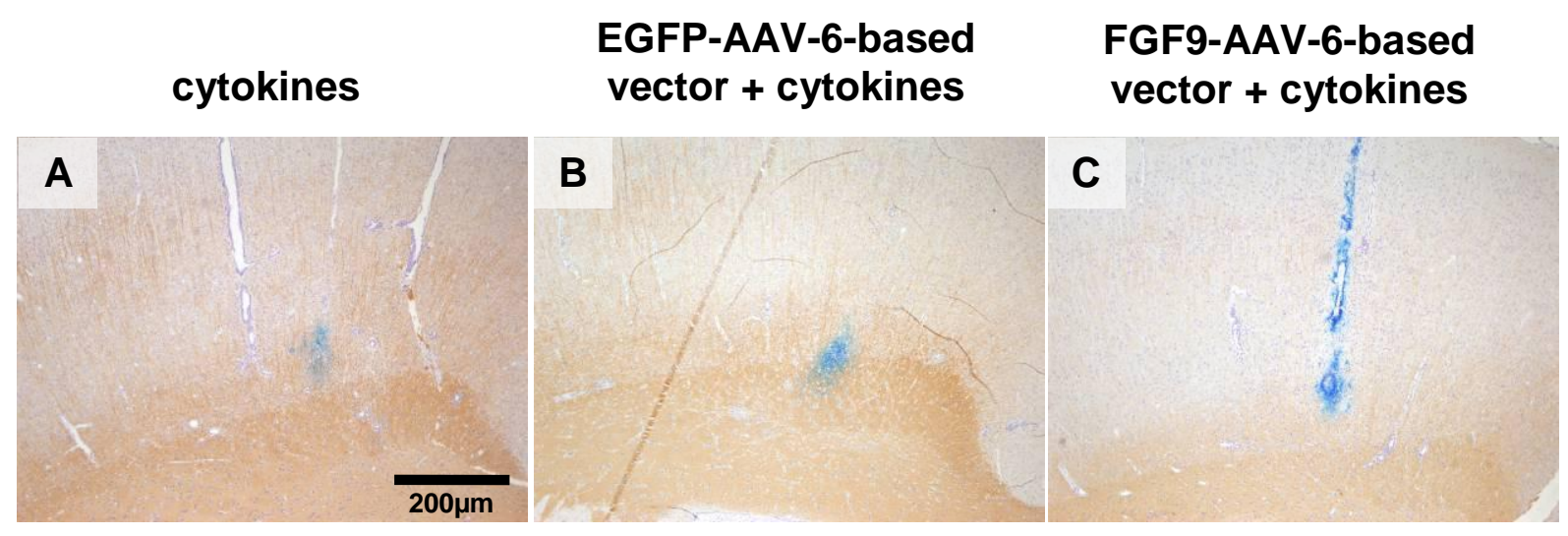

Figure 3.33: Rat focal EAE 3 days after injection of cytokines alone (A), cytokines with EGFPAAV-6-based vector (C) and cytokines with FGF9-AAV-6-based vector (E). No obvious DM in MBP staining was detected in all 3 experimental groups.

Double labeling studies for GFAP and Ki67 revealed that part of the astrocytes were positive for the proliferation marker in animals injected with cytokines and FGF9AAV-6-based vector (Fig. 3.35 A). Conspicuous aggregates of NogoA positive cells were found near the injection site in these animals (Fig. 3.35 B).

As my results above indicated that FGF9 might be pro-inflammatory, the influence of FGF9-AAV-6-based vector on immunized rats without cytokine injection was determined. Interestingly, 10 days after injection a prominent area of DM was visible despite of the lack of a focal cytokine injection (Fig. 3.36 A). The activation pattern of astrocytes (Fig. $3.36 \mathrm{~B}$ ) and the increase in numbers of astroglial and oligodendroglial cells was similar (Fig. 3.36 C-F) to the combined injection with cytokines and FGF9-AAV-6-based vector. 


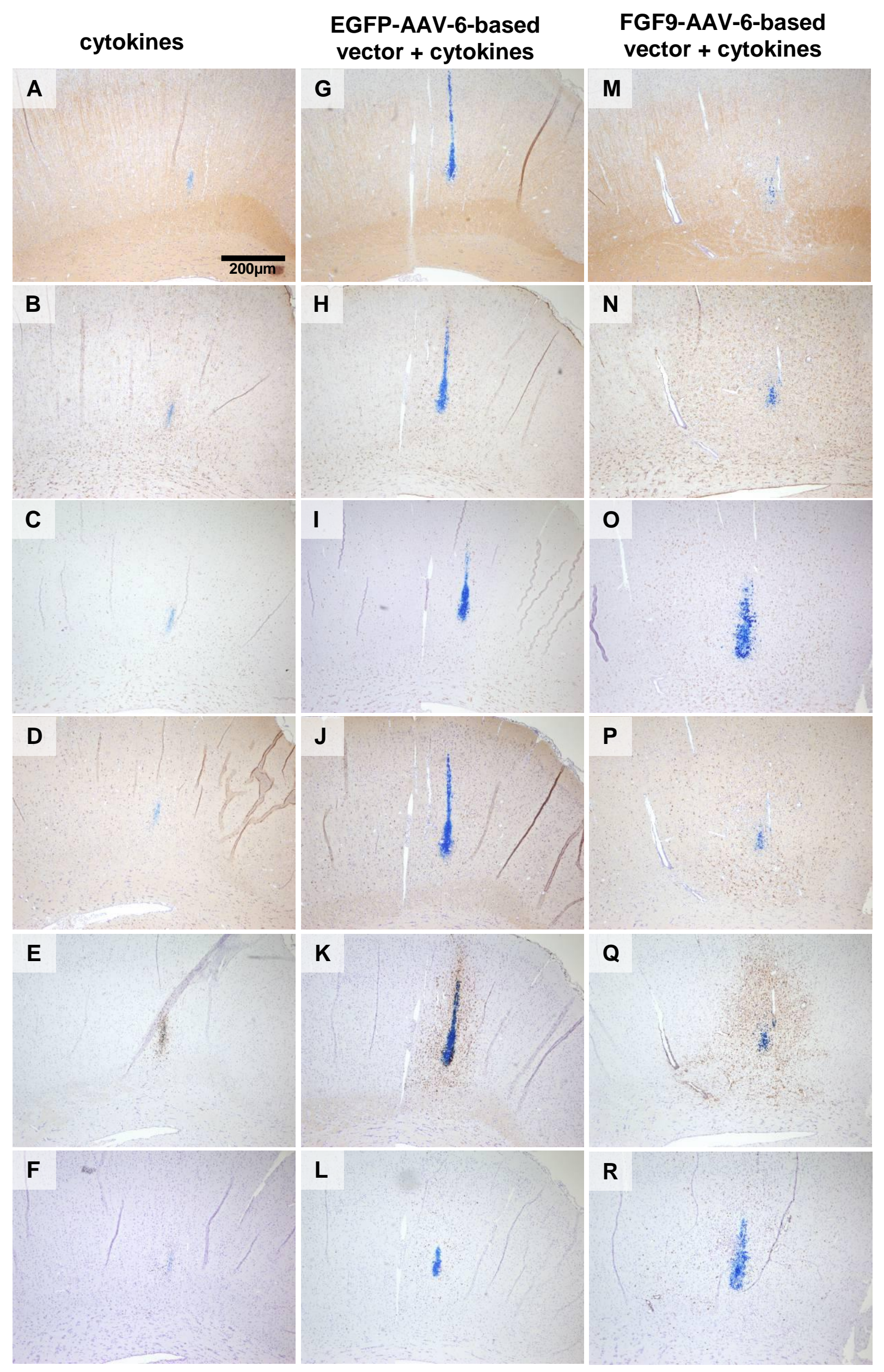


Figure 3.34: Rat focal EAE 10 days after injection of cytokines (A-F), cytokines with EGFP-AAV6-based vector (G-L) and cytokines with FGF9-AAV-6-based vector (M-R). Only in animals injected with FGF9-AAV-6-based vector small foci of DM occur (A, G, M; MBP). Furthermore, with FGF9 the area of activated astrocytes was conspicuously larger than in the other experimental groups (N; GFAP). Numbers of Olig2 and NogoA positive cells were increased $(\mathrm{O}, \mathrm{P})$. The highest numbers of ED1 positive cells were also detected in animals injected with cytokines and FGF9-AVV-6-based vector (Q). Additionally they showed an increase in Ki67 positive cells (R). A, G, M: MBP (brown); B, H, N: GFAP; C, I, O: Olig2; D, J, P: NogoA; E, K, Q: ED1; F, L, R: Ki67.
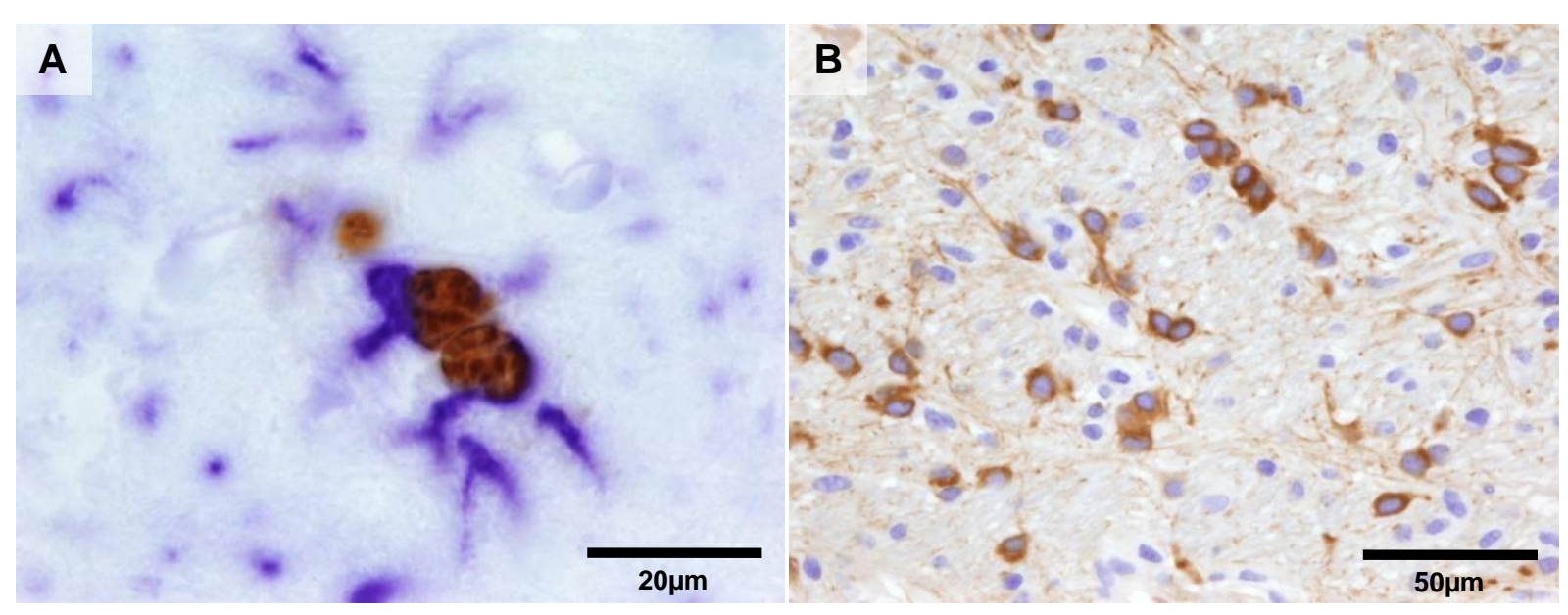

Figure 3.35: Astrocytic and oligodendroglial proliferation in the rat focal EAE model after intracerebral injection with cytokines and FGF9-AAV-6-based vector. (A) Cells double positive for GFAP (blue) and Ki67 (brown). (B) Increased density of NogoA (brown) positive cells near the injection site.

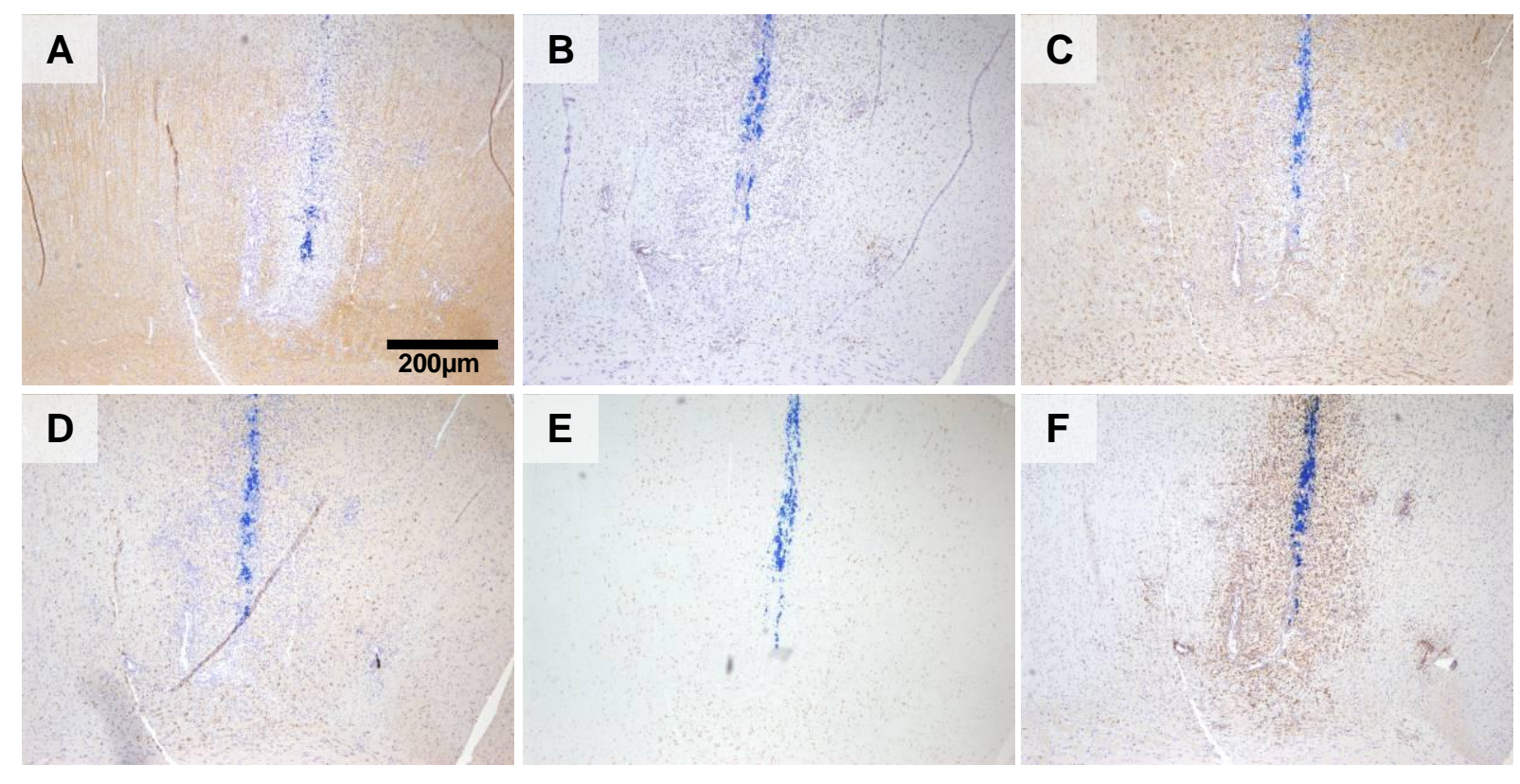

Figure 3.36: Rat focal EAE 10 days after injection of FGF9-AAV-6-based vector alone. Prominent DM (A; MBP) was visible after 10 days. Additionally, a high number of proliferating cells is present in this area (B; Ki67). In C (GFAP) astrocytes appear extremely activated. Mature oligodendrocytes (D; NogoA) and OPCs (E; Olig2) were increased in the area of activated astrocytes. Additionally, a large area of activated microglia/macrophages (E; ED1) is detected 


\section{$4 \quad$ Discussion}

$\mathrm{NMO}$ is an inflammatory demyelinating disease that preferentially targets the optic nerves and the spinal cord. Previously thought to be a variant of MS, the discovery of an autoantibody directed against AQP4 in $75 \%$ of the NMO but not in MS patients defined NMO as an independent disorder. The aim of the present thesis was to characterize in more detail the mechanisms underlying the tissue damage induced by recombinant patient derived antibodies directed against AQP4 in animal models. The antibodies were reconstructed from CSF plasma cells of an NMO patient (Bennett et al., 2009) and injected stereotactically (focal NMO model) or intravenously (systemic EAE/NMO model) into Lewis rats. Specifically, I addressed the following questions: How are astrocytes destroyed by AQP4 recombinant antibodies? Why do oligodendrocytes die after AQP4 recombinant antibody application? Do astrocytes and oligodendrocytes repopulate the lesion differently?

The second part of my thesis studied the DM and RM potential of FGF9 in vivo in cuprizone-induced DM in the mouse and focal EAE in the rat.

\subsection{Modeling Neuromyelitis optica in vivo}

The stereotactic injection of $\mathrm{rAb}-53$ leads to a rapid loss of astrocytes, which is followed by a loss of oligodendrocytes. Astrocyte depleted areas harbor APP positive axons, complement and Ig depositions. The early inflammatory infiltrate is composed of PMNs (Fig. 3.5). Thus the early lesion pathology has striking similarities with human NMO lesions as described by Lucchinetti and coworkers (2002) and Roemer and coworkers (2007). Stereotactically generated lesions slightly differ from NMO-like lesions induced by i.v. application of the antibody (Fig. 3.2). In the latter case animals immunized with a short MBP peptide, which opens the BBB for the antibody in the spinal cord of the animals. The lesion distribution of i.v. injected animals therefore follows a perivascular pattern and the inflammatory lesion is mainly composed of mononuclear cells (Fig. 3.12). In addition, i.v. injected animals suffer from EAE induced motor deficits, which increase by the injection of rAb-53 (Fig. 3.11). This is in contrast to stereotactic injections of rAb-53 into the motor cortex, which do not 
become noticeable in the EAE scoring system. Compared to previously published models which transferred NMO serum antibodies into rats with acute T-cell-mediated EAE (Bradl et al., 2009), the lesion size obtained by the transfer of recombinant antibodies is more impressive and most likely reflects the amount of pathogenic $\mathrm{rAb}$ 53 transferred.

Oligodendrocyte loss can be depicted early after lesion induction by immunostainings against NogoA/p25 (Fig. 3.3 and 1.2) and was also described by Parratt and Prineas (2010) for human NMO. Astrocytes repopulate the lesion quickly 1 week after injection, whereas oligodendrocyte depletion remains prominent at this time point (Fig. 3.7). The reason for this longer lasting oligodendrocyte depletion is still obscure, however, the number of oligodendrocyte precursor cells is also considerably diminished after rAb-53 injection (Fig. $3.2 \mathrm{C}$ ) and thus the basic source of oligodendrocyte replenishment. The massive loss of OPCs could explain the delay in oligodendroglial repopulation of astrocyte depleted lesions that is the prerequisite for remylination in other experimental and human CNS diseases, such as MS (Prineas et al., 1989; Raine et al., 1981; Robinson et al., 1998). Furthermore, astrocyte loss clearly affects the RM outcome in a number of animal models, and astrocytes might provide necessary guiding cues and trophic support for oligodendrocytes (Talbott et al., 2005).

In my experiments I took advantage of a recombinant antibody against AQP4 instead of serum from NMO patients (Bennett et al., 2009). This offered the unique opportunity to modify antibody effector functions by mutating the Fc region. In this regard, the injection of rAb-53_K322A, the AQP4 specific antibody deficient for complement activation caused a significantly smaller area of astrocyte depletion than the wt rAb-53 (Fig. 3.21). Therefore astrocyte depletion by this recombinant antibody is significantly dependent on its ability to activate complement. This is in line with human NMO lesions, which stain positively for complement factors, suggesting that complement mediated astrocyte loss might be relevant in the human disorder as well (Lucchinetti et al., 2002). Complement activation generates the small complement fragments $\mathrm{C} 5 \mathrm{a}$ and $\mathrm{C} 3 \mathrm{a}$, which are potent peptide mediators of inflammation, and attract phagocytic cells including PMNs to the lesion site. 
Ablating the ability of rAb-53 to activate natural killer (NK) cells (rAb-53_K326W) slightly reduced its ability to destroy astrocytes (Fig. 3.21) but rAb-53 devoid of both effector functions (rAb-53_L234A) was not less effective than AQP4 specific antibodies without complement activation properties. This clearly argues against a relevant contribution of NK cells to $\mathrm{rAb}-53$ mediated astrocyte loss. However, mutated recombinant antibodies hardly differed from wt rAb-53 regarding oligodendrocyte loss in astrocyte depleted areas (Fig. 3.22).

In summary, both NMO models mimic the pathology of human NMO lesions including loss of oligodendroglial cells. Furthermore, my findings underpin the role of complement for astrocyte depletion.

\subsubsection{Mechanism of oligodendroglial cell death in vivo and in vitro}

I have consistently observed the rapid death of OPCs and oligodendrocytes after rAb-53 injection, and both cell types are AQP4 negative. This is in line with the results of Marignier and coworkers (2010), who found oligodendrocyte death in cell culture experiments and ex vivo optic nerve explants when exposed to IgG from NMO patients. The authors postulated that a disruption of glutamate homeostasis and elevated extracellular glutamate levels that occurred as a consequence of astrocyte death, led to oligodendrocyte demise via binding to NMDA receptors. I also considered glutamate or ATP mediated excitotoxicity as the most likely mechanism of oligodendrocyte death happening within few hours after $\mathrm{rAb}-53$ mediated astrocyte loss in my animal model. Oligodendrocytes express the ionotropic glutamate receptors amino-3-hydroxy-5-methyl 4-isoxazolepropionic acid, kainate and NMDA throughout most developmental stages (Karadottir et al., 2005; Matute et al., 2007a). In addition, NMDA receptor antagonists as well as kainate and AMPA receptor blockers have been beneficial in inflammatory demyelinating animal models for MS (Pitt et al., 2000; Smith et al., 2000; Wallstrom et al., 1996). In animals injected with rAb-53 I was, however, unable to show a convincing rescue from oligodendrocyte death by NMDA receptor antagonists (D-AP5 and MK-801)(Fig. 3.9), which I studied first due to their higher affinity for glutamate relative to AMPA receptors (Karadottir et al., 2005). Glutamate is taken up by astrocytes via the glutamate transporter 1 (GLT1 or EAAT-2) and usually quickly converted into glutamine by the astroglial glutamine synthetase (Danbolt, 2001; Parpura and Haydon, 2009). Therefore the increase in 
extracellular glutamate concentrations after rAb-53 mediated astrocyte lysis in vivo may rather be caused by a reduced number of astrocytes capable of glutamate uptake via GLT1 than by the liberation of stored glutamate vesicles. Interestingly, the extent of oligodendrocyte loss in the grey matter following rAb-53 injection (Fig. 3.13) is larger than in the white matter. This may be due to higher glutamate levels in the grey matter (Lehre et al., 1995). I am currently blocking AMPA/kainate glutamatergic receptors by CNQX and NBQX to test whether these substances are able to prevent oligodendrocyte death after $\mathrm{rAb}-53$ mediated astrocyte loss. Kainate receptors have also been implicated in sensitizing oligodendrocytes to complement attacks (Alberdi et al., 2006), which might be relevant considering the fact that the effector function of my rAb-53 is complement dependent. The second excitatory neurotransmitter which can damage oligodendrocytes if present in excess is ATP. If present in unusually high concentrations ATP can bind to $P_{2} X_{7}$ receptors on oligodendrocytes. The prolonged activation of $\mathrm{P}_{2} \mathrm{X}_{7}$ receptors on oligodendrocytes results in the overload of the cytosol with $\mathrm{Na}^{+}, \mathrm{Ka}^{+}$and $\mathrm{Ca}^{2+}$ (North, 2002), caspase 3 activation and finally cell

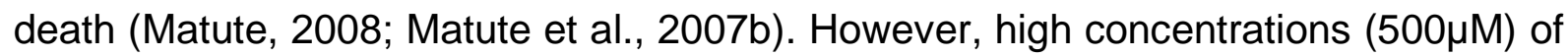
the $\mathrm{P}_{2} \mathrm{X}_{7}$ receptor antagonists oATP or BBG did not clearly prevent or ameliorate oligodendrocyte loss after stereotactic injection of rAb-53 (Fig. 3.10).

Taken together, I could not show a convincing rescue of oligodendroglial cells by injection of NMDA or $\mathrm{P}_{2} \mathrm{X}_{7}$ receptor antagonists. To clarify their role further experiments and counts of the cell numbers have to be performed. Experiments applying AMPA/kainate receptor antagonists are ongoing. Furthermore, I hypothesize that multiple mechanisms might operate in parallel to induce the observed rapid loss of oligodendroglial cells. To prove this hypothesis a combinatorial treatment with both, NMDA and $\mathrm{P}_{2} \mathrm{X}_{7}$, receptor antagonists will be performed in vivo and in vitro.

\subsubsection{Kidney pathology in the EAE/NMO model}

Since AQP4 is not only expressed in astrocytes of the CNS but can also be detected in the collecting ducts of the kidney, I screened my animals for kidney pathology following i.v. antibody transfer. The inner medulla of the kidney was infiltrated by macrophages $30 \mathrm{hrs}$ after antibody transfer and these collecting ducts appeared swollen with visible cell detachments (Fig. 3.19+18). Urine analysis demonstrated high protein concentrations as well as cell debris. Pohl and coworkers (2011) who 
transferred AQP4 specific T-cells together with NMO IgG induced an interstitial nephritis, while my kidney pathology localized rather to the collecting ducts.

From my results I conclude that kidney pathology could be relevant in NMO. Despite the fact that AQP4 is also expressed in the human kidney (Mobasheri et al., 2007) a clinically apparent involvement of the kidneys has so far not been revealed in NMO patients.

\subsection{Effect of astrocytic overexpression of FGF9}

RM is a complex process which, if successful, restores myelin to demyelinated axons. At first, OPCs have to be recruited to the demyelinated area by pro-migratory factors released from reactive astrocytes and inflammatory cells (Ffrench-Constant and Raff, 1986). OPCs will populate the demyelinated area and have to differentiate into myelinating oligodendrocytes. This involves axon engagement and the formation of a myelin sheath (Franklin and Ffrench-Constant, 2008). The differentiation of OPCs to remyelinating oligodendrocytes is the most vulnerable phase of RM and in most animal models RM fails at this stage.

In the second part of my thesis I have studied the effect of FGF9 in de- and remyelinating animal models by its overexpression in astrocytes. FGF9 was one of the molecules identified to be upregulated in demyelinated compared to remyelinated MS lesions in a microarray study performed by the group of Edgar Meinl (MaxPlanck-Institute for Neurobiology, Munich; unpublished data). I therefore speculated that FGF9 might be an inhibitor of OPC differentiation.

Overexpression of FGF9 in astrocytes was obtained by constructing an AAV-6-based vector which expressed FGF9 under the control of the GFAP promoter. For control experiments, the same vector expressing EGFP under the GFAP promoter was constructed demonstrating EGFP expression predominantly in astrocytes after stereotactic injections into naive animals (Fig. 3.27 D). FGF9 overexpression reached its maximum 7-10 days after injection (Fig. 3.27 A) and its effect was studied in two experimental paradigms. 


\subsubsection{Overexpression of FGF9 in the cuprizone mouse model of toxic DM and in a model of antibody-mediated focal inflammatory DM in the rat}

Feeding mice with cuprizone for 5 weeks leads to oligodendrocyte death and the DM of white matter tracts, which is best depicted in the corpus callosum. If cuprizone feeding is stopped, demyelinated lesions become quickly remyelinated and this process is completed in around 2 weeks (Matsushima and Morell, 2001). I stereotactically injected the FGF9-AAV-6-based vector or EGFP-AAV-6-based vector 4 weeks after initiation of the cuprizone feeding. Animals were sacrificed 1 week later (while still on cuprizone diet) to address the influence of FGF9 on DM or 10 days later (already three days on normal diet) to study if FGF9 promotes or inhibits RM. I could not detect any differences with respect to DM or RM in animals which received the FGF9-AAV-6-based vector compared to EGFP-AAV-6-based vector or PBS injected controls (Fig. 3.28+3.30). However, the number of OPCs and oligodendrocytes was significantly higher in the FGF9 expressing group already 7 days after injection, and mitotic oligodendrocytes could easily be detected (Fig 3.28 [small insert]). The rise in OPC and oligodendrocyte numbers became even more prominent at the second time point (Fig. 3.30 and Fig. 3.31). Furthermore a change in oligodendrocyte morphology was obvious with larger oligodendrocytes in the FGF9 group compared to controls. GFAP upregulation and astrogliosis in the FGF9-AAV-6based vector injected group was detectable at day 7 after injection and even more prominent 3 days later.

FGF9 overexpression in a model of stereotactically induced focal anti-MOG antibody mediated inflammatory DM led to similar results. In this model FGF9-AAV-6-based vector or EGFP-AAV-6-based vector were injected concomitantly with proinflammatory cytokines into MOG primed rats (Merkler et al., 2006). As further controls, I analyzed naïve animals injected with both AAV-6-based vector constructs but no additional cytokines (Fig. 3.32). In the focal EAE model, DM is most important three days after injection, and RM is completed after around 2 weeks. Unfortunately, all animals sacrificed at day 3 did not show demyelinated areas, which might be due to insufficient anti-MOG antibody titers after immunization (Fig. 3.33). While my focal experiments were therefore limited with regard to the study of de- or RM properties of 
FGF9 overexpression, astrocyte and oligodendrocyte proliferation was detectable in all FGF9-AAV-6-based vector injected animals but not in controls (Fig. 3.34). Furthermore, at day 10 FGF9-AAV-6-based vector injected animals as well as animals which were injected with FGF9-AAV-6-based vector in combination with inflammatory cytokines harboured small foci of DM, whereas control animals with EGFP-AAV6-based vector and inflammatory cytokines did not (Fig 3.35 A). In addition, injection of FGF9-AAV-6-based vector in immunized rats without cytokines showed a proinflammatory effect with infiltrating macrophage/activated microglia (Fig. 3.36 F) accompanied by a prominent demyelinated area.

My results confirm the mitogenic potential of FGF9 on the oligodendrocyte lineage and resemble results obtained by overexpressing PDGF $\alpha$ in astrocytes using GFAPPDGF $\alpha$ mice (Woodruff et al., 2004). These mice, although having significantly higher numbers of OPCs, did not show more efficient RM following lysolecithin injection or cuprizone mediated DM. Therefore, in both models OPC recruitment does not seem to be the rate limiting factor for RM. While Cohen and Chandross (2000) suggested that FGF9 might suppress oligodendrocyte differentiation of primary rat oligodendrocytes in vitro, RM in vivo was not inhibited in the cuprizone model by FGF9 overexpression. Astrocytes responded to FGF9 overexpression in vivo by an upregulation of GFAP, which is in contrast to the results obtained by Lum and coworkers (2009a) who reported the strong inhibition of GFAP positive astrocyte differentiation from adult neural progenitor cells. I can detect small areas of DM following the stereotactic injection of FGF9-AAV-6-based vector in the focal EAE model at day 10, which needs to be addressed in further experiments. The most likely explanation is a disruption of the BBB which allows demyelinating antibodies to enter the CNS parenchyma. FGF9 might thus also increase the local inflammatory response.

In summary I have established the stable expression of FGF9 in astrocytes by using an AAV-6-based vector. I can confirm the mitogenic potential of FGF9 on oligodendrocytes and astrocytes in vivo. FGF9 overexpression however did not influence remyelination in my experimental models. Further experiments have to address the potential proinflammatory activity and clarify if FGF9 modulates BBB functions. 
Alberdi, E., Sanchez-Gomez, M.V., Torre, I., Domercq, M., Perez-Samartin, A., Perez-Cerda, F., and Matute, C. (2006). Activation of kainate receptors sensitizes oligodendrocytes to complement attack. J Neurosci 26, 3220-3228.

Amiry-Moghaddam, M., and Ottersen, O.P. (2003). The molecular basis of water transport in the brain. Nat Rev Neurosci 4, 991-1001.

Barbieri, F., and Buscaino, G.A. (1989). Neuromyelitis optica in the elderly. Acta Neurol (Napoli) 11, 247-251.

Bennett, J.L., Lam, C., Kalluri, S.R., Saikali, P., Bautista, K., Dupree, C., Glogowska, M., Case, D., Antel, J.P., Owens, G.P., et al. (2009). Intrathecal pathogenic antiaquaporin-4 antibodies in early neuromyelitis optica. Ann Neurol 66, 617-629.

Bradl, M., Misu, T., Takahashi, T., Watanabe, M., Mader, S., Reindl, M., Adzemovic, M., Bauer, J., Berger, T., Fujihara, K., et al. (2009). Neuromyelitis optica: pathogenicity of patient immunoglobulin in vivo. Ann Neurol 66, 630-643.

Brambilla, R., Bracchi-Ricard, V., Hu, W.H., Frydel, B., Bramwell, A., Karmally, S., Green, E.J., and Bethea, J.R. (2005). Inhibition of astroglial nuclear factor kappaB reduces inflammation and improves functional recovery after spinal cord injury. $J$ Exp Med 202, 145-156.

Brum, D.G., Barreira, A.A., dos Santos, A.C., Kaimen-Maciel, D.R., Matiello, M., Costa, R.M., Deghaide, N.H., Costa, L.S., Louzada-Junior, P., Diniz, P.R., et al. (2010). HLA-DRB association in neuromyelitis optica is different from that observed in multiple sclerosis. Mult Scler 16, 21-29.

Bsibsi, M., Persoon-Deen, C., Verwer, R.W., Meeuwsen, S., Ravid, R., and Van Noort, J.M. (2006). Toll-like receptor 3 on adult human astrocytes triggers production of neuroprotective mediators. Glia 53, 688-695.

Bush, T.G., Puvanachandra, N., Horner, C.H., Polito, A., Ostenfeld, T., Svendsen, C.N., Mucke, L., Johnson, M.H., and Sofroniew, M.V. (1999). Leukocyte infiltration, neuronal degeneration, and neurite outgrowth after ablation of scar-forming, reactive astrocytes in adult transgenic mice. Neuron 23, 297-308.

Cohen, R.I., and Chandross, K.J. (2000). Fibroblast growth factor-9 modulates the expression of myelin related proteins and multiple fibroblast growth factor receptors in developing oligodendrocytes. J Neurosci Res 61, 273-287.

Cree, B.A., Reich, D.E., Khan, O., De Jager, P.L., Nakashima, I., Takahashi, T., BarOr, A., Tong, C., Hauser, S.L., and Oksenberg, J.R. (2009). Modification of Multiple Sclerosis Phenotypes by African Ancestry at HLA. Arch Neurol 66, 226-233.

Danbolt, N.C. (2001). Glutamate uptake. Prog Neurobiol 65, 1-105.

Davis, R., Thiele, E., Barnes, P., and Riviello, J.J., Jr. (1996). Neuromyelitis optica in childhood: case report with sequential MRI findings. J Child Neurol 11, 164-167. 
Dreyfus, C.F., Dai, X., Lercher, L.D., Racey, B.R., Friedman, W.J., and Black, I.B. (1999). Expression of neurotrophins in the adult spinal cord in vivo. J Neurosci Res $56,1-7$.

Faulkner, J.R., Herrmann, J.E., Woo, M.J., Tansey, K.E., Doan, N.B., and Sofroniew, M.V. (2004). Reactive astrocytes protect tissue and preserve function after spinal cord injury. J Neurosci 24, 2143-2155.

Ffrench-Constant, C., and Raff, M.C. (1986). The oligodendrocyte-type-2 astrocyte cell lineage is specialized for myelination. Nature 323, 335-338.

Fortin, D., Rom, E., Sun, H., Yayon, A., and Bansal, R. (2005). Distinct fibroblast growth factor (FGF)/FGF receptor signaling pairs initiate diverse cellular responses in the oligodendrocyte lineage. J Neurosci 25, 7470-7479.

Franklin, R.J., and Ffrench-Constant, C. (2008). Remyelination in the CNS: from biology to therapy. Nat Rev Neurosci 9, 839-855.

Gard, A.L., Burrell, M.R., Pfeiffer, S.E., Rudge, J.S., and Williams, W.C., 2nd (1995). Astroglial control of oligodendrocyte survival mediated by PDGF and leukemia inhibitory factor-like protein. Development 121, 2187-2197.

Ghezzi, A., Bergamaschi, R., Martinelli, V., Trojano, M., Tola, M.R., Merelli, E., Mancardi, L., Gallo, P., Filippi, M., Zaffaroni, M., et al. (2004). Clinical characteristics, course and prognosis of relapsing Devic's Neuromyelitis Optica. J Neurol 251, 47-52.

Graber, D.J., Levy, M., Kerr, D., and Wade, W.F. (2008). Neuromyelitis optica pathogenesis and aquaporin 4. J Neuroinflammation 5, 22.

Grimm, D., Kay, M.A., and Kleinschmidt, J.A. (2003). Helper virus-free, optically controllable, and two-plasmid-based production of adeno-associated virus vectors of serotypes 1 to 6 . Mol Ther 7, 839-850.

He, F., and Sun, Y.E. (2007). Glial cells more than support cells? Int J Biochem Cell Biol 39, 661-665.

Huang, J.Y., and Chuang, J.I. (2010). Fibroblast growth factor 9 upregulates heme oxygenase-1 and gamma-glutamylcysteine synthetase expression to protect neurons from 1-methyl-4-phenylpyridinium toxicity. Free Radic Biol Med 49, 1099-1108.

Huang, J.Y., Hong, Y.T., and Chuang, J.I. (2009). Fibroblast growth factor 9 prevents MPP+-induced death of dopaminergic neurons and is involved in melatonin neuroprotection in vivo and in vitro. J Neurochem 109, 1400-1412.

Jack, C.S., Arbour, N., Manusow, J., Montgrain, V., Blain, M., McCrea, E., Shapiro, A., and Antel, J.P. (2005). TLR signaling tailors innate immune responses in human microglia and astrocytes. J Immunol 175, 4320-4330.

Jarius, S., Hoffmann, L., Clover, L., Vincent, A., and Voltz, R. (2008). CSF findings in patients with voltage gated potassium channel antibody associated limbic encephalitis. J Neurol Sci 268, 74-77. 
Kanda, T., Iwasaki, T., Nakamura, S., Ueki, A., Kurokawa, T., Ikeda, K., and Mizusawa, H. (1999). FGF-9 is an autocrine/paracrine neurotrophic substance for spinal motoneurons. Int J Dev Neurosci 17, 191-200.

Karadottir, R., Cavelier, P., Bergersen, L.H., and Attwell, D. (2005). NMDA receptors are expressed in oligodendrocytes and activated in ischaemia. Nature 438, 11621166.

Kim, H.J., Park, H.Y., Kim, E., Lee, K.S., Kim, K.K., Choi, B.O., Kim, S.M., Bae, J.S., Lee, S.O., Chun, J.Y., et al. (2010). Common CYP7A1 promoter polymorphism associated with risk of neuromyelitis optica. Neurobiol Dis 37, 349-355.

Kim, J.H., Park, J.A., Lee, S.W., Kim, W.J., Yu, Y.S., and Kim, K.W. (2006). Bloodneural barrier: intercellular communication at glio-vascular interface. J Biochem Mol Biol 39, 339-345.

Kugler, S., Lingor, P., Scholl, U., Zolotukhin, S., and Bahr, M. (2003). Differential transgene expression in brain cells in vivo and in vitro from AAV-2 vectors with small transcriptional control units. Virology 311, 89-95.

Kuhlmann, T., Miron, V., Cui, Q., Wegner, C., Antel, J., and Bruck, W. (2008). Differentiation block of oligodendroglial progenitor cells as a cause for remyelination failure in chronic multiple sclerosis. Brain 131, 1749-1758.

Lefkowitz, D., and Angelo, J.N. (1984). Neuromyelitis optica with unusual vascular changes. Arch Neurol 41, 1103-1105.

Lehre, K.P., Levy, L.M., Ottersen, O.P., Storm-Mathisen, J., and Danbolt, N.C. (1995). Differential expression of two glial glutamate transporters in the rat brain: quantitative and immunocytochemical observations. J Neurosci 15, 1835-1853.

Lennon, V.A., Kryzer, T.J., Pittock, S.J., Verkman, A.S., and Hinson, S.R. (2005). IgG marker of optic-spinal multiple sclerosis binds to the aquaporin-4 water channel. J Exp Med 202, 473-477.

Lennon, V.A., Wingerchuk, D.M., Kryzer, T.J., Pittock, S.J., Lucchinetti, C.F., Fujihara, K., Nakashima, I., and Weinshenker, B.G. (2004). A serum autoantibody marker of neuromyelitis optica: distinction from multiple sclerosis. Lancet 364, 21062112.

Lin, Y., Chen, L., Lin, C., Luo, Y., Tsai, R.Y., and Wang, F. (2009). Neuron-derived FGF9 is essential for scaffold formation of Bergmann radial fibers and migration of granule neurons in the cerebellum. Dev Biol 329, 44-54.

Linington, C., Bradl, M., Lassmann, H., Brunner, C., and Vass, K. (1988). Augmentation of demyelination in rat acute allergic encephalomyelitis by circulating mouse monoclonal antibodies directed against a myelin/oligodendrocyte glycoprotein. Am J Pathol 130, 443-454.

Lucchinetti, C.F., Mandler, R.N., McGavern, D., Bruck, W., Gleich, G., Ransohoff, R.M., Trebst, C., Weinshenker, B., Wingerchuk, D., Parisi, J.E., et al. (2002). A role 
for humoral mechanisms in the pathogenesis of Devic's neuromyelitis optica. Brain $125,1450-1461$.

Lum, M., Turbic, A., Mitrovic, B., and Turnley, A.M. (2009a). Fibroblast growth factor9 inhibits astrocyte differentiation of adult mouse neural progenitor cells. J Neurosci Res 87, 2201-2210.

Lum, M., Turbic, A., Mitrovic, B., and Turnley, A.M. (2009b). Fibroblast growth factor9 inhibits astrocyte differentiation of adult mouse neural progenitor cells. J Neurosci Res 87, 2201-2210.

Ma, T., Yang, B., Gillespie, A., Carlson, E.J., Epstein, C.J., and Verkman, A.S. (1997). Generation and phenotype of a transgenic knockout mouse lacking the mercurial-insensitive water channel aquaporin-4. J Clin Invest 100, 957-962.

Malik, J.M., Shevtsova, Z., Bahr, M., and Kugler, S. (2005). Long-term in vivo inhibition of CNS neurodegeneration by Bcl-XL gene transfer. Mol Ther 11, 373-381.

Mandler, R.N., Ahmed, W., and Dencoff, J.E. (1998). Devic's neuromyelitis optica: a prospective study of seven patients treated with prednisone and azathioprine. Neurology 51, 1219-1220.

Mandler, R.N., Davis, L.E., Jeffery, D.R., and Kornfeld, M. (1993). Devic's neuromyelitis optica: a clinicopathological study of 8 patients. Ann Neurol 34, 162168.

Manley, G.T., Fujimura, M., Ma, T., Noshita, N., Filiz, F., Bollen, A.W., Chan, P., and Verkman, A.S. (2000). Aquaporin-4 deletion in mice reduces brain edema after acute water intoxication and ischemic stroke. Nat Med 6, 159-163.

Marignier, R., Nicolle, A., Watrin, C., Touret, M., Cavagna, S., Varrin-Doyer, M., Cavillon, G., Rogemond, V., Confavreux, C., Honnorat, J., et al. (2010). Oligodendrocytes are damaged by neuromyelitis optica immunoglobulin $G$ via astrocyte injury. Brain 133, 2578-2591.

Matiello, M., Schaefer-Klein, J.L., Hebrink, D.D., Kingsbury, D.J., Atkinson, E.J., and Weinshenker, B.G. (2011). Genetic analysis of aquaporin-4 in neuromyelitis optica. Neurology $77,1149-1155$.

Matsushima, G.K., and Morell, P. (2001). The neurotoxicant, cuprizone, as a model to study demyelination and remyelination in the central nervous system. Brain Pathol $11,107-116$.

Matsushita, T., Matsuoka, T., Isobe, N., Kawano, Y., Minohara, M., Shi, N., Nishimura, Y., Ochi, H., and Kira, J. (2009). Association of the HLA-DPB1*0501 allele with anti-aquaporin-4 antibody positivity in Japanese patients with idiopathic central nervous system demyelinating disorders. Tissue Antigens 73, 171-176.

Matute, C. (2008). P2X7 receptors in oligodendrocytes: a novel target for neuroprotection. Mol Neurobiol 38, 123-128. 
Matute, C., Alberdi, E., Domercq, M., Sanchez-Gomez, M.V., Perez-Samartin, A., Rodriguez-Antiguedad, A., and Perez-Cerda, F. (2007a). Excitotoxic damage to white matter. J Anat 210, 693-702.

Matute, C., Torre, I., Perez-Cerda, F., Perez-Samartin, A., Alberdi, E., Etxebarria, E., Arranz, A.M., Ravid, R., Rodriguez-Antiguedad, A., Sanchez-Gomez, M., et al. (2007b). P2X(7) receptor blockade prevents ATP excitotoxicity in oligodendrocytes and ameliorates experimental autoimmune encephalomyelitis. J Neurosci 27, 95259533.

Medzhitov, R. (2007). Recognition of microorganisms and activation of the immune response. Nature 449, 819-826.

Merkler, D., Ernsting, T., Kerschensteiner, M., Bruck, W., and Stadelmann, C. (2006). A new focal EAE model of cortical demyelination: multiple sclerosis-like lesions with rapid resolution of inflammation and extensive remyelination. Brain 129, 1972-1983.

Misu, T., Fujihara, K., Kakita, A., Konno, H., Nakamura, M., Watanabe, S., Takahashi, T., Nakashima, I., Takahashi, H., and Itoyama, Y. (2007). Loss of aquaporin 4 in lesions of neuromyelitis optica: distinction from multiple sclerosis. Brain 130, 1224-1234.

Misu, T., Fujihara, K., Nakamura, M., Murakami, K., Endo, M., Konno, H., and Itoyama, Y. (2006). Loss of aquaporin-4 in active perivascular lesions in neuromyelitis optica: a case report. Tohoku J Exp Med 209, 269-275.

Mobasheri, A., Marples, D., Young, I.S., Floyd, R.V., Moskaluk, C.A., and Frigeri, A. (2007). Distribution of the AQP4 water channel in normal human tissues: protein and tissue microarrays reveal expression in several new anatomical locations, including the prostate gland and seminal vesicles. Channels (Austin) 1, 29-38.

Moe, S.E., Sorbo, J.G., Sogaard, R., Zeuthen, T., Petter Ottersen, O., and Holen, T. (2008). New isoforms of rat Aquaporin-4. Genomics 91, 367-377.

Mothet, J.P., Parent, A.T., Wolosker, H., Brady, R.O., Jr., Linden, D.J., Ferris, C.D., Rogawski, M.A., and Snyder, S.H. (2000). D-serine is an endogenous ligand for the glycine site of the N-methyl-D-aspartate receptor. Proc Natl Acad Sci U S A 97, 4926-4931.

Nakamura, S., Todo, T., Haga, S., Aizawa, T., Motoi, Y., Ueki, A., Kurokawa, T., and Ikeda, K. (1997). Motor neurons in human and rat spinal cord synthesize fibroblast growth factor-9. Neurosci Lett 221, 181-184.

Nakamura, S., Todo, T., Motoi, Y., Haga, S., Aizawa, T., Ueki, A., and Ikeda, K. (1999). Glial expression of fibroblast growth factor-9 in rat central nervous system. Glia 28, 53-65.

North, R.A. (2002). Molecular physiology of P2X receptors. Physiol Rev 82, 10131067.

Oertle, T., van der Haar, M.E., Bandtlow, C.E., Robeva, A., Burfeind, P., Buss, A., Huber, A.B., Simonen, M., Schnell, L., Brosamle, C., et al. (2003). Nogo-A inhibits 
neurite outgrowth and cell spreading with three discrete regions. J Neurosci 23, 5393-5406.

Padmawar, P., Yao, X., Bloch, O., Manley, G.T., and Verkman, A.S. (2005). K+ waves in brain cortex visualized using a long-wavelength $\mathrm{K}+$-sensing fluorescent indicator. Nat Methods 2, 825-827.

Parpura, V., and Haydon, P.G. (2009). Astrocytes in (patho)physiology of the nervous system (New York, Springer).

Parratt, J.D., and Prineas, J.W. (2010). Neuromyelitis optica: a demyelinating disease characterized by acute destruction and regeneration of perivascular astrocytes. Mult Scler 16, 1156-1172.

Pitt, D., Werner, P., and Raine, C.S. (2000). Glutamate excitotoxicity in a model of multiple sclerosis. Nat Med 6, 67-70.

Pohl, M., Fischer, M.T., Mader, S., Schanda, K., Kitic, M., Sharma, R., Wimmer, I., Misu, T., Fujihara, K., Reindl, M., et al. (2011). Pathogenic T cell responses against aquaporin 4. Acta Neuropathol 122, 21-34.

Prineas, J.W., Kwon, E.E., Goldenberg, P.Z., Ilyas, A.A., Quarles, R.H., Benjamins, J.A., and Sprinkle, T.J. (1989). Multiple sclerosis. Oligodendrocyte proliferation and differentiation in fresh lesions. Lab Invest 61, 489-503.

Raine, C.S., Scheinberg, L., and Waltz, J.M. (1981). Multiple sclerosis. Oligodendrocyte survival and proliferation in an active established lesion. Lab Invest 45, 534-546.

Rash, J.E., Davidson, K.G., Yasumura, T., and Furman, C.S. (2004). Freeze-fracture and immunogold analysis of aquaporin-4 (AQP4) square arrays, with models of AQP4 lattice assembly. Neuroscience 129, 915-934.

Reuss, B., Hertel, M., Werner, S., and Unsicker, K. (2000). Fibroblast growth factors5 and -9 distinctly regulate expression and function of the gap junction protein connexin43 in cultured astroglial cells from different brain regions. Glia 30, 231-241.

Robinson, S., Tani, M., Strieter, R.M., Ransohoff, R.M., and Miller, R.H. (1998). The chemokine growth-regulated oncogene-alpha promotes spinal cord oligodendrocyte precursor proliferation. J Neurosci 18, 10457-10463.

Roemer, S.F., Parisi, J.E., Lennon, V.A., Benarroch, E.E., Lassmann, H., Bruck, W., Mandler, R.N., Weinshenker, B.G., Pittock, S.J., Wingerchuk, D.M., et al. (2007). Pattern-specific loss of aquaporin-4 immunoreactivity distinguishes neuromyelitis optica from multiple sclerosis. Brain 130, 1194-1205.

Saadoun, S., Papadopoulos, M.C., Watanabe, H., Yan, D., Manley, G.T., and Verkman, A.S. (2005). Involvement of aquaporin-4 in astroglial cell migration and glial scar formation. J Cell Sci 118, 5691-5698. 
Schell, M.J., Molliver, M.E., and Snyder, S.H. (1995). D-serine, an endogenous synaptic modulator: localization to astrocytes and glutamate-stimulated release. Proc Natl Acad Sci U S A 92, 3948-3952.

Smith, T., Groom, A., Zhu, B., and Turski, L. (2000). Autoimmune encephalomyelitis ameliorated by AMPA antagonists. Nat Med 6, 62-66.

Sofroniew, M.V., and Vinters, H.V. (2010). Astrocytes: biology and pathology. Acta Neuropathol 119, 7-35.

Talbott, J.F., Loy, D.N., Liu, Y., Qiu, M.S., Bunge, M.B., Rao, M.S., and Whittemore, S.R. (2005). Endogenous Nkx2.2+/Olig2+ oligodendrocyte precursor cells fail to remyelinate the demyelinated adult rat spinal cord in the absence of astrocytes. Exp Neurol 192, 11-24.

Terris, J., Ecelbarger, C.A., Marples, D., Knepper, M.A., and Nielsen, S. (1995). Distribution of aquaporin-4 water channel expression within rat kidney. Am J Physiol 269, F775-785.

Todo, T., Kondo, T., Nakamura, S., Kirino, T., Kurokawa, T., and Ikeda, K. (1998). Neuronal localization of fibroblast growth factor-9 immunoreactivity in human and rat brain. Brain Res 783, 179-187.

van Loo, G., De Lorenzi, R., Schmidt, H., Huth, M., Mildner, A., Schmidt-Supprian, M., Lassmann, H., Prinz, M.R., and Pasparakis, M. (2006). Inhibition of transcription factor NF-kappaB in the central nervous system ameliorates autoimmune encephalomyelitis in mice. Nat Immunol 7, 954-961.

Wallstrom, E., Diener, P., Ljungdahl, A., Khademi, M., Nilsson, C.G., and Olsson, T. (1996). Memantine abrogates neurological deficits, but not CNS inflammation, in Lewis rat experimental autoimmune encephalomyelitis. J Neurol Sci 137, 89-96.

Wells, T. (1998). Vesicular osmometers, vasopression secretion and aquaporin-4: a new mechanism for osmoreception? Mol Cell Endocrinol 136, 103-107.

Wingerchuk, D.M., Hogancamp, W.F., O'Brien, P.C., and Weinshenker, B.G. (1999). The clinical course of neuromyelitis optica (Devic's syndrome). Neurology 53, 11071114.

Wingerchuk, D.M., Lennon, V.A., Lucchinetti, C.F., Pittock, S.J., and Weinshenker, B.G. (2007a). The spectrum of neuromyelitis optica. Lancet Neurol 6, 805-815.

Wingerchuk, D.M., Lennon, V.A., Pittock, S.J., Lucchinetti, C.F., and Weinshenker, B.G. (2006). Revised diagnostic criteria for neuromyelitis optica. Neurology 66, 14851489.

Wingerchuk, D.M., Pittock, S.J., Lucchinetti, C.F., Lennon, V.A., and Weinshenker, B.G. (2007b). A secondary progressive clinical course is uncommon in neuromyelitis optica. Neurology 68, 603-605.

Wingerchuk, D.M., and Weinshenker, B.G. (2003). Neuromyelitis optica: clinical predictors of a relapsing course and survival. Neurology 60, 848-853. 
Wingerchuk, D.M., and Weinshenker, B.G. (2008). Neuromyelitis optica. Curr Treat Options Neurol 10, 55-66.

Wolburg, H. (1995). Orthogonal arrays of intramembranous particles: a review with special reference to astrocytes. J Hirnforsch 36, 239-258.

Woodruff, R.H., Fruttiger, M., Richardson, W.D., and Franklin, R.J. (2004). Plateletderived growth factor regulates oligodendrocyte progenitor numbers in adult CNS and their response following CNS demyelination. Mol Cell Neurosci 25, 252-262.

Zephir, H., Fajardy, I., Outteryck, O., Blanc, F., Roger, N., Fleury, M., Rudolf, G., Marignier, R., Vukusic, S., Confavreux, C., et al. (2009). Is neuromyelitis optica associated with human leukocyte antigen? Mult Scler 15, 571-579.

Zonta, M., Sebelin, A., Gobbo, S., Fellin, T., Pozzan, T., and Carmignoto, G. (2003). Glutamate-mediated cytosolic calcium oscillations regulate a pulsatile prostaglandin release from cultured rat astrocytes. J Physiol 553, 407-414. 


\section{Appendix}

\section{A1 Solutions and reagents}

Phosphate buffered saline (PBS):

$\begin{array}{ll}\text { PBS (Dulbecco, Biochrom AG) } & 9.55 \mathrm{~g} \\ \text { distilled water } & 1000 \mathrm{ml}\end{array}$

$1 \mathrm{M}$ sulfuric acid:

96\% sulfuric acid (Merck)

$51.1 \mathrm{ml}$

distilled water

$448.9 \mathrm{ml}$

4\% paraformaldehyde (PFA):

PFA (Merck, Germany)

$40 \quad 9$

1.0 M sodium hydroxide

1 drop

10-fold PBS

$100 \mathrm{ml}$

distilled water

$900 \mathrm{ml}$

adjust to $\mathrm{pH} 7.3$

0.05\% Triton containing phosphate buffered saline (PBST):

PBS $1000 \mathrm{ml}$

Triton X-100 (MP Biomedicals) $500 \mu \mathrm{l}$

BME-medium:

inactivated horse serum

$50 \mathrm{ml}$

Pen/Strep

$5 \mathrm{ml}$

Glutamax

$5 \mathrm{ml}$

BME-medium

$460 \mathrm{ml}$

filter sterile

Super-Sato medium:

B27-Supplement

$2 \mathrm{ml}$

Glutamax

$1 \mathrm{ml}$

Pen/Strep

$1 \mathrm{ml}$

inactivated horse serum

$1 \mathrm{ml}$ 
Pyruvate (1.1\%)

$1 \mathrm{ml}$

Triiodthyronin

$10 \mu \mathrm{l}$

L-Thyroxin

$13 \mu \mathrm{l}$

DMEM

$94 \mathrm{ml}$

filter sterile

0.5\% PLL-stock solution:

Poly-L-lysin

19

PBS (sterile)

$200 \mathrm{ml}$

$\underline{0.01 \% \text { PLL-ready-to-use solution: }}$

PLL-stock solution

$10 \mathrm{ml}$

PBS

$500 \mathrm{ml}$

\section{H \& E staining:}

$1 \%$ acid rinse:

$\begin{array}{lll}30 \% \text { hydrochloric acid } & 2 & \mathrm{ml} \\ \text { 100\% Isopropyl alcohol (Merck) } & 198 & \mathrm{ml}\end{array}$

$1 \%$ eosin solution:

Eosin-G Certistain@ (Merck) $2 \mathrm{ml}$

$70 \%$ isopropyl alcohol

$198 \mathrm{ml}$

(filter before use)

\section{LFB-PAS staining:}

LFB solution:

LFB

19

95\% EtOH

$1000 \mathrm{ml}$

acetic acid

$5 \mathrm{ml}$

\section{Bielschowsky silver staining:}

20\% silver nitrate solution:

$\begin{array}{lll}\text { silver nitrate (Roth) } & 10 & \mathrm{~g} \\ \text { distilled water } & 50 & \mathrm{ml}\end{array}$

Developer stock solution: 
37\% formalin (Merck)

Citric acid (Merck)

$65 \%$ nitric acid (Merck)

distilled water
$20 \mathrm{ml}$

$0.5 \mathrm{~g}$

2 drops

$100 \mathrm{ml}$

$10 \mathrm{~g}$

$500 \mathrm{ml}$

\section{Pappenheim staining:}

May-Grünwald solution:

$50 \mathrm{ml}$

Giemsa solution

Giemsa

$1 \mathrm{ml}$

PBS

$49 \mathrm{ml}$

\section{Immunohistochemistry:}

$10 \mathrm{mM}$ citric acid buffer:

$\begin{array}{ll}\text { citric acid (Merck) } & 2.1 \mathrm{~g} \\ \text { distilled water } & 1000 \mathrm{ml} \\ \text { adjust to } \mathrm{pH} 6 & \end{array}$

Tris-ethylenediaminetetraacetic acid (Tris-EDTA) buffered saline:

Trizma base (Sigma)

$1.21 \mathrm{~g}$

$0.1 \mathrm{M}$ EDTA $1 \mathrm{ml}$

distilled water $1000 \mathrm{ml}$

adjust to $\mathrm{pH} 9$

$\underline{0.2 \% \text { casein block: }}$

Tropix (Applied Biosystems)

29

PBS $1000 \mathrm{ml}$

Tween 20 $1 \mathrm{ml}$

heat Tropix/PBS to $50^{\circ} \mathrm{C}$ until it dissolves

1.0M Tris/HCl stock solution:

trizma base (Sigma) 
$30 \%$ hydrochloric acid

sodium chloride (Merck)

distilled water fill up to

adjust to $\mathrm{pH} 7.5$
$400 \mathrm{ml}$

$170 \mathrm{~g}$

$1000 \mathrm{ml}$

TBS working solution (washing buffer for immunohistochemistry):

sodium chloride (Merck)

1.0M Tris/ $\mathrm{HCl}$

adjust to $\mathrm{pH} 7.5$
$9 \quad 9$

$50 \mathrm{ml}$

Fast Blue working solution:

$0.1 \mathrm{M}$ Tris ( $\mathrm{pH} 8.2)$

$49 \mathrm{ml}$

naphtol-ASMX-phosphate (Sigma) $1 \mathrm{ml}$

1.0M levimasole (Sigma)

$50 \mu \mathrm{l}$

Fast Red TR salt (Sigma)

$0.025 \mathrm{~g}$

filter before use

Fast Red working solution:

$\begin{array}{lll}0.1 \mathrm{M} \text { Tris (pH 8.2) } & 49 & \mathrm{ml} \\ \text { naphtol-ASMX-phosphate (Sigma) } & 1 & \mathrm{ml} \\ \text { 1.0M levimasole (Sigma) } & 50 & \mathrm{\mu l} \\ \text { Fast Red TR salt (Sigma) } & 0.05 & \mathrm{~g} \\ \text { filter before use } & & \end{array}$

3,3'-diaminobenzidine tetrachloride (DAB) working solution:

$25 \mathrm{mg}$

PBS

$50 \mathrm{ml}$

$30 \%$ hydrogen peroxidase (Merck) $\quad 30 \quad \mu \mathrm{l}$

$1 \%$ nickel ammonium sulfate solution:

$\begin{array}{lll}\text { nickel ammonium sulfate } & 0.1 & \mathrm{~g} \\ \text { distilled water } & 10 & \mathrm{ml}\end{array}$

4-nitro blue tetrazolium chloride (NBT) stock solution: 


$\begin{array}{lll}70 \% \text { N,N-dimethylformamide } & 1 & \mathrm{ml} \\ \text { NBT (Roche) } & 100 & \mathrm{mg}\end{array}$

5-bromo-4-chloro-3-indolyl phosphate (BCIP) stock solution:

$70 \% \mathrm{~N}, \mathrm{~N}$-dimethylformamide $\quad 1 \mathrm{ml}$

(Sigma)

BCIP (Roche)

$50 \quad \mathrm{mg}$

NBT/BCIP buffer:

1.0M Tris (Sigma-Aldrich) $\quad 100 \mathrm{ml}$

$5.0 \mathrm{M} \mathrm{NaCl}$ (Merck) $\quad 20 \mathrm{ml}$

$1.0 \mathrm{M} \mathrm{MgCl}$ (Merck) $\quad 50 \mathrm{ml}$

distilled water $\quad 100 \mathrm{ml}$

NBT/BCIP working solution:

NBT stock solution $\quad 225 \mu \mathrm{l}$

BCIP stock solution $\quad 175 \mu \mathrm{l}$

NBT/BCIP buffer $\quad 50 \mathrm{ml}$

1xIST working solution:

Tailing buffer

$14 \mu \mathrm{l}$

$\mathrm{CoCl}$

$2.8 \mu \mathrm{l}$

Digoxigenin-DNA

$1.4 \mu \mathrm{l}$

terminal transferase

$0.7 \mu \mathrm{l}$

distilled water

$51.1 \mu \mathrm{l}$

\section{In situ hybridization:}

Hybridization-mix/section:

$\begin{array}{lll}\text { dextran } & 10 & \mu \mathrm{l} \\ \text { 20x SSC } & 5 & \mu \mathrm{l} \\ \text { deion. formamide } & 25 & \mu \mathrm{L} \\ \text { Lachs-Sp.-DNA } & 1 & \mu \mathrm{l} \\ \text { 2\% SDS } & 0.5 & \mu \mathrm{l} \\ \text { PLP probe } & 3 & \mu \mathrm{l}\end{array}$


$\mathrm{H}_{2} \mathrm{O}$ DEPC

$5.5 \mu \mathrm{l}$

acetic acid
agarose
ammonium chloride
ammonium hydroxide
brilliant blue G
BME
boric acid
citric acid
Chloroacetate-esterase

chloral hydrate

copper sulphate

CytotoxOne

DAB

D-AP5

DePeX

diethyl ether

DMEM

EDTA

Eosin-G

$\mathrm{EtOH}$

formalin

Giemsa

$\mathrm{H}_{2} \mathrm{O}_{2}$

HBSS

IFA

IFN-Y
Merck KgaA, Darmstadt, Germany

StarPure, StarLab GmbH, Ahrensberg, Germany

Pharm Lyse, 10x conc., BD Biosciences, Heidelberg, Germany

Merck KgaA, Darmstadt, Germany

Sigma-Aldrich Chemie GmbH, Steinheim, Germany

Gibco, Invitrogen, Darmstadt, Germany

Roth, Karlsruhe, Germany

Merck KgaA, Darmstadt, Germany

Naphthol Chloroacetate esterase kit, detection of

polymorphnuclear cells, Sigma-Aldrich Chemie $\mathrm{GmbH}$, Steinheim, Germany

Merck KgaA, Darmstadt, Germany

Merck KgaA, Darmstadt, Germany

membrane integrity assay, Promega, Madison, WI, USA

Sigma-Aldrich Chemie GmbH, Steinheim, Germany

Tocris bioscience, Biozol, Eching, Germany

VWR international, Darmstadt, Germany

Sigma-Aldrich Chemie GmbH, Steinheim, Germany

High Glucose (4.5g/l), w/ L-Glutamine, sodium pyruvate, Gibco, Invitrogen, Darmstadt, Germany

Roth, Karlsruhe, Germany

CertiStain Merck KgaA, Darmstadt, Germany

absolute Merck KgaA, Darmstadt, Germany

Merck KgaA, Darmstadt, Germany

Merck KgaA, Darmstadt, Germany

Merck KgaA, Darmstadt, Germany

Gibco, Karlsruhe, Germany

Sigma-Aldrich Chemie GmbH, Steinheim, Germany

PeproTech, London, UK 
isopropanol

ketamine

LFB

lithium carbonate

nickel ammonium sulfate

Mayer's hemalum

May-Grünwald solution

methanol

MK-801

monastral blue

mounting-medium

mycobacteria tuberculosis H37 RA, Difco Laboratories, Augsburg, Germany

$\mathrm{NaCl}$

$0.9 \% \mathrm{NaCl}$ solution

naphtyl ethylene

oATP

Proteinase $\mathrm{K}$

TUNEL

Tween 20
Merck KgaA, Darmstadt, Germany

B. Braun, Germany

Merck KgaA, Darmstadt, Germany

Inresa, Freiburg, Germany

BDH Laboratory supplies, VWR Int. Ltd., Poole, UK

Merck KgaA, Darmstadt, Germany

Merck KgaA, Darmstadt, Germany

Merck KgaA, Darmstadt, Germany

Merck KgaA, Darmstadt, Germany

Merck KgaA, Darmstadt, Germany

Sigma-Aldrich Chemie GmbH, Steinheim, Germany

Copper (II) phthalocyaninetetrasulfonic acid tetrasodium salt, Sigma-Aldrich Chemie GmbH, Steinheim, Germany

Fluorescence-mounting-medium, Dako

Merck KgaA, Darmstadt, Germany

Sigma-Aldrich Chemie GmbH, Steinheim, Germany

Sigma-Aldrich Chemie GmbH, Steinheim, Germany

Roche Diagnostics GmbH, Mannheim, Germany

Merck KgaA, Darmstadt, Germany 24-well plates

12-well plates

96-well plates

Analysis $^{\mathrm{TM}}$

cell strainer

combi screen 3 plus

cytospin centrifuge

ELISA reader

fluorescence reader
Greiner Bio-One GmbH, Frickenhausen, Germany

Greiner Bio-One $\mathrm{GmbH}$, Frickenhausen, Germany

Greiner Bio-One GmbH, Frickenhausen, Germany

software, University of Wisconsin, Madison, WI

$(40 \mu \mathrm{m}) \mathrm{BD}$ Biosciences, Heidelberg, Germany

analyticon, Lichtenfels, Germany

Shandon Cytospin 4, Thermo Scientific, Dreieich, Germany

Model 680, Biorad, Munich, Germany

Safire, Tecan, Mainz, Germany 


$\begin{array}{ll}\begin{array}{l}\text { glass slides } \\ \text { glass capillary }\end{array} & \begin{array}{l}\text { Menzel, Braunschweig, Germany } \\ \text { GraphPad }\end{array} \\ \text { incubator } & \text { software, San Diego, CA, USA } \\ \text { micro osmometer } & \text { Cellstar, Nunc GmbH, Wiesbaden, Germany } \\ \text { type OM806, Vogel/Löser, Berlin, Germany } \\ \text { microscope } & \text { (light), BX41, Olympus, Europa GmbH, Hamburg, } \\ & \text { Germany } \\ \text { microtome } & \text { Leica, Wetzlar, Germany } \\ \text { microwave } & \text { Bosch, Gerlingen-Schillerhohe, Germany } \\ \text { Neubauer } & \text { (counting chamber) Brand GmbH \& Co KG, Wertheim, } \\ & \text { Germany } \\ \text { sonicator } & \text { SonoPuls, Bandelin Electronics, Berlin, Germany } \\ \text { stereotactic device } & \text { Stoelting Co, IL, USA } \\ \text { tissue processor } & \text { TP 1020, Leica, Wetzlar, Germany }\end{array}$




\section{Acknowledgements}

I thank Prof. Dr. med. Wolfgang Brück for giving me the opportunity to work and write my thesis in the Department of Neuropathology.

I mostly would like to thank my supervisor and mentor Prof. Dr. med. Christine Stadelmann-Nessler for her great support, listening to my needs, excellent guidance, helpful discussions, never ending patience and giving me the chance to work independently.

Furthermore, I would like to thank Stefan Nessler for his great theortical support and helpful corrections.

I like to thank my thesis committee Prof. Dr. Michael Müller and Prof. Dr. Dr. Hannelore Ehrenreich for their helpful comments and ideas.

I wish to thank Prof. Dr. Jeffrey Bennett for providing me his rAbs.

I am grateful for the great support from our technicians, Jasmin, Katja, Angela, Brigitte, Uta and Heidi.

Great thanks to my colleagues and friends in the Department of Neuropathology. Because of the nice and friendly atmosphere in the department work was not difficult.

Special thanks to my family. My parents and siblings supported me over all years. Thanks to my girls (Eva and Christina) for the nice evenings (beer and wine).

Finally, I would like to thank Martin for his endless love, patience and his sensitivity. 


\section{Curriculum vitae}

\section{Personal data}

$\begin{array}{ll}\text { Name } & \text { Claudia Veronika Wrzos } \\ \text { Date/Place of birth: } & \text { April } 17^{\text {th }}, 1982 \text { in Pszczyna (Pleß), Poland } \\ \text { Nationality: } & \text { German } \\ \text { Contact: } & \text { University Medical Center } \\ & \text { Institute of Neuropathology } \\ & \text { Robert-Koch-Straße 40 } \\ & \text { 37099 Göttingen } \\ & \text { claudia.wrzos@gmx.de }\end{array}$

\section{Education and Qualifications}
06/2008 - present Institute of Neuropathology, University Medical Center Göttingen, Germany
Ph.D. student
Program of the DFG Research Center Molecular
Physiology of the Brain (CMPB), Gottingen Graduate School for Neurosciences and Molecular Biosciences (GGNB)

$2008-2010 \quad$ Ph.D. project in part funded by CMPB

03.2007-03.2008 Georg-August-University Göttingen, Germany

Women's representative for the faculty of Biology

10/2002 - 01/2008 Georg-August-University Göttingen, Germany

Studies in biology

Degree: Diplom $(1,7)$

2002

Jakob-Grimm-Schule, Rotenburg a. d. Fulda

Abitur 


\section{Professional experience}

06.2008 - present

03.2007-22.01.2008

$10 / 11.2006$

09.2007

02/03.2007

09.2006
Institute of Neuropathology, University Medical Center Göttingen, Germany

Ph.D. student

Research project: "The role of astrocytes for oligodendrocyte death and remyelination"

Supervisor: Prof. Dr. med. Christine Stadelmann-Nessler

Georg-August-University Göttingen, Germany

Department of Neurobiology

Diploma thesis: „Circadiane Beeinflussung

neurosekretorischer Nervenzellen bei Invertebraten."

Supervisor: Prof. Dr. Ralf Heinrich

Georg-August-University Göttingen, Germany

Department of Neurobiology

Voluntary internship (Electrophysiology)

Georg-August-University Göttingen, Germany

Department of Neurobiology

Student assistant in teaching

Topic: „Psychophysik des Sehens und Verhalten (Reflexe und Lernen).“

Georg-August-University Göttingen, Germany

Department of Neurobiology

Student assistant in teaching

Topic: „Nerven-, Sinnes- und Muskelphysiologie.“

Georg-August-University Göttingen, Germany

Department of Neurobiology

Student assistant in teaching 
Topic: „Psychophysik des Sehens und Verhalten (Reflexe und Lernen).“

09.2005

Georg-August-University Göttingen, Germany

Department of Neurobiology

Student assistant in teaching

Topic: „Psychophysik des Sehens und Verhalten (Reflexe und Lernen).“

\section{Publications}

2011

Boretius S, Escher A, Dallenga T, Wrzos C, Tammer R, Brück W, Nessler S, Frahm J, Stadelmann C.

„Assessment of lesion pathology in a new model of MS by multiparametric MRI and DTI."

Neuroimage 2012; 59:2678-88

\section{Talks}

2010

Wrzos C., Bennett J., Brück W., Nessler S., Stadelmann C.

"Astrocyte depletion by anti-AQP4 antibodies in vivo" NeuroWind, Motzen (Berlin), Germany

2010

Wrzos C., Bennett J., Brück W., Nessler S., Stadelmann C.

"Repair of selective astrocyte depletion in the rodent brain."

ECTRIMS 2010, Gothenburg, Sweden 


\section{Poster presentations}

2011

2010

2010

2009

2009
Wrzos C., Bennett J., Brück W., Nessler S., Stadelmann C.

"Loss and repair of selective astrocyte depletion followed by oligodendrocyte cell loss in vitro and in vivo." $10^{\text {th }}$ European meeting on Glial cells in health and disease, Prague, Chech Republic

Wrzos C., Bennett J., Hemmer B., Brück W., Nessler S., Stadelmann C.

"Repair of selective astrocyte depletion in the rodent brain."

NeuroWoche, Mannheim, Germany

Wrzos C., Bennett J., Hemmer B., Brück W., Nessler S., Stadelmann C.

"Selective astrocyte depletion in the rodent brain" Inflammation in Brain Disease (NeurlnfNet), Odense, Denmark

Wrzos C., Bennett J., Hemmer B., Brück W., Nessler S., Stadelmann C.

"Selective astrocyte depletion in the rodent brain" German Society of Neuropathology and Neuroanatomy, Dusseldorf, Germany

Wrzos C., Bennett J., Hemmer B., Brück W., Nessler S., Stadelmann C.

"Selective astrocyte depletion in the rodent brain" Ph.D. program GGNB Science Day, Gottingen, Germany 